\title{
Quasiorder lattices of universal algebras
}

\author{
Ph.D. Thesis \\ Gergő Gyenizse
}

\begin{abstract}
Advisor: Miklós Maróti
Doctoral School of Mathematics and Computer Science

Department of Algebra and Number Theory, Bolyai Institute, University of Szeged
\end{abstract}

Szeged, 2018 


\section{Contents}

1 Introduction $\quad 2$

2 Basics 4

2.1 Lattices of compatible relations . . . . . . . . . . . . 4

2.2 The term clone and the polynomial clone . . . . . . . . 6

2.3 Varieties, quasivarieties and Mal'tsev classes . . . . . . . . . 7

2.4 Semidistributivity, lower and upper bounded lattices . . . . . . 10

3 Lattice representations with DCC posets 14

3.1 Representation of finite lattices . . . . . . . . . . . . . 15

3.2 Representation of arbitrary lattices . . . . . . . . . . . . . 19

4 Overview of tame congruence theory 24

4.1 Minimal algebras and tame quotients . . . . . . . . . . . 24

4.2 Types in the congruence lattice . . . . . . . . . . 26

4.3 Description of some Mal'tsev classes . . . . . . . . . . 29

5 Quasiorder types in finite algebras $\quad 32$

5.1 Definition of types for quasiorder quotients . . . . . . . . . 32

5.2 Properties of different types . . . . . . . . . . . . . 35

5.3 Types in a quasiorder lattice $\ldots \ldots \ldots \ldots \ldots$

6 Varieties omitting certain types $\quad 46$

6.1 Congruence modular varieties . . . . . . . . . . . . . 47

6.2 Varieties omitting types 1 and $2 \ldots \ldots \ldots \ldots \ldots$

6.3 Varieties omitting 1,2 , and $5 \ldots \ldots \ldots \ldots \ldots$

6.4 Quasiorder meet semidistributivity . . . . . . . . . . . 67

$\begin{array}{lll}7 & \text { Summary } & 69\end{array}$

8 Összefoglaló $\quad 73$ 


\section{Chapter 1}

\section{Introduction}

Modern algebra's most essential concept is arguably the congruence relation. The study of any algebraic structure having a nontrivial congruence can be started by studying its typically simpler factor. Moreover, for the classical algebraic structures the congruence is determined by a subalgebra, again, typically a simpler structure. The natural structure formed by the congruences of an algebra is a lattice (or rather, in the case of infinitely many congruences, an algebraic lattice).

On the other hand, certain classes of structures-notably, lattices themselveshave congruences that are not determined by a particular subalgebra (or any particular congruence class). Still, the congruences of these structures are of great importance, but for lattices and semilattices in particular, there is an other kind of binary relation that is more closely associated with the structure: the so-called natural order. There is no general definition for what makes an order natural (except by untrustworthy ideologues), but a common requirement seems to be that the order should be compatible with the operations of the algebra. A notable exception is the natural order on regular semigroups (see Chapter 6 of [13]), which is not generally compatible with the semigroup multiplication.

The obvious common generalization of congruences (compatible, reflexive, transitive, symmerical binary relations) and compatible orders (the same, with antisymmetry instead of symmetry) are quasiorders (the same, without symmetry). The quasiorders of an algebra (unlike the compatible orders) form a lattice.

Tame congruence theory, developed in the 1970s and 1980s, became a strong tool in the study of universal algebra and related fields such as complexity theory. It provides connections between some transitivity properties of the polynomial clone of a finite algebra, the congruence lattices in the variety generated by this algebra, and the Mal'tsev classes this variety belongs to, among other things. The main purpose of this thesis is to bring quasiorder lattices into this mix.

As a quasiorder can be factored to a symmetrical and an antisymmetrical part, it is natural to first study parts of the quasiorder lattice that only contain 
compatible orders (these may be called "order lattices", though this is a slightly confusing term). Such a study can be found in Chapter 3, which is essentially a reprint of [15] (with some definitions moved to Chapter 2). As it turns out, order lattices can be completely general on infinite underlying sets, but must have a particular property on finite ones: they have a nontrivial distributive homomorphic image, unless they themselves are trivial. In other words, they satisfy a "solvability for distributivity" property (actually, they also satisfy a somewhat stronger property). Instead of finiteness of the underlying set, one may consider only orders satisfying some finiteness condition. The most natural of these is that the orders do not contain infinite chains. The same restrictions apply to lattices of such orders as when the finiteness of the underlying set is assumed. The chapter is mostly about a weaker condition: only the DCC is required of the orders. In this case, it turns out there are still restrictions for the lattice, but not as strong as in the case of finite underlying set. This chapter is essentially independent of the subsequent ones.

A main goal of this thesis is to study connections between the congruence and the quasiorder lattice of a finite algebra. The closest connections are proved for algebras in congruence modular varieties, in which case the two lattices generate the same lattice variety (Theorem 6.7). This is a common generalization of two theorems in the paper co-written by the author and his advisor ([18]). Theorem 6.7 is from [16]. There are further (if weaker) connections between the congruence and quasiorder lattices of an algebra, and particularly the sets of all congruence and all quasiorder lattices of algebras of locally finite varieties.

Chapter 4 provides a very brief summary of tame congruence theory, only to provide the definitions and theorems needed for the last two chapters. It contains no proofs, safe for those concerning quasiorders instead of congruences. The chapter is not meant as an introduction to the topic, but it should be enough for a reader desiring to verify the subsequent proofs.

The heart of tame congruence theory is the types assigned to any covering pair in a congruence lattice of a finite algebra. This assignment has a more-orless natural counterpart for the quasiorder lattice, which is described in Chapter 5 (based on [17]). Chapter 6 describes the properties of quasiorder lattices in varieties that omit certain types. This part is based on [18] and [17]. Certain parts of the former are changed for the sake of internal cohesion. In particular, Theorem 19 of [18] is essentially the same as Corollary 6.21 of this thesis, and is proved there without using quasiorder types.

To understand this thesis, the reader should have some very basic knowledge about universal algebra and lattice theory. A single course in each is probably sufficient. 


\section{Chapter 2}

\section{Basics}

In this thesis, an algebra always means what is sometimes called a universal algebra: a pair $\mathbf{A}=\left(A,\left\{f_{i}: i \in I\right\}\right)$, where $A$ is a set, $I$ is an index set, and for all $i \in I, f_{i}$ is an operation of some arity on $A$. The $f_{i}$ are called the basic operations of the algebra, the set $I$ the set of operations symbols. Usually, the operation symbol and the corresponding basic operation- $i$ and $f_{i}$-will not be distinguished by notation.

\subsection{Lattices of compatible relations}

Definition 2.1. A $k$-ary relation $R$ is compatible with the $n$-ary relation $f$ (both given on the same set $A$ ) if any choice of $\left(a_{i, j}\right)_{n \times k} \in A^{n \times k}$ satisfying

$$
\left(a_{i, 1}, \ldots, a_{i, k}\right) \in R
$$

for all $1 \leq i \leq n$ also satisfies

$$
\left(f\left(a_{1,1}, \ldots, a_{n, 1}\right), \ldots, f\left(a_{1, k}, \ldots, a_{n, k}\right)\right) \in R .
$$

A relation $R$ on $A$ is compatible with the algebra $\mathbf{A}$ (with the same underlying set) if it is compatible with all of its basic operations.

It is obvious that the intersection of any number of compatible relations of the same arity is again a compatible relation. Therefore, for any algebra and any natural $k$ the set of $k$-ary compatible relations form a (complete) lattice. In this thesis we are concerned only with the $k=2$ case, acknowledging that higher arity compatible relations are central to the study of clones.

Definition 2.2. The elements of a binary relation $\rho$ are called edges. If $(a, b)$ is an edge, $a$ is called the source, $b$ the target of this edge. In the case $a=b$, the edge is called a loop. A $\rho$-path is a sequence of edges $c_{0}, \ldots, c_{s}$ such that for each $0 \leq i<s,\left(c_{i}, c_{i+1}\right)$ is an edge of $\rho$.

Instead of $(a, b) \in \rho$ we will often write $a \stackrel{\rho}{\rightarrow} b$. 
Definition 2.3. The set of all reflexive and compatible binary relations of an algebra $\mathbf{A}$ is denoted by $R(\mathbf{A})$. The symmetric elements of $R(\mathbf{A})$ are called tolerances, the transitive elements quasiorders, and the symmetric and transitive ones congruences, denoted by $\operatorname{Tol} \mathbf{A}$, Quo $\mathbf{A}$, and Con $\mathbf{A}$ respectively. All of these sets are closed to intersection, hence they form lattices with respect to inclusion, the lattices are denoted the same way.

For a binary relation $\rho, \operatorname{Tr} \rho$ denotes the transitive closure of $\rho$.

It is important to note that while Quo $\mathbf{A}$ is a subset of $R(\mathbf{A})$, and Con $\mathbf{A}$ is a subset of Tol $\mathbf{A}$, they are generally not sublattices, for it can happen, for example, that the smallest tolerance containing two congruences (i.e. their join in the tolerance lattice) is not a congruence. (An example can be easily constructed in an algebra with empty set of basic operations.) On the other hand, Con $\mathbf{A} \leq$ Quo $\mathbf{A}$ and Tol $\mathbf{A} \leq R(\mathbf{A})$ are always true.

Definition 2.4. For a set $A$, Pre $A$ denotes the set (and lattice) of all preorders, i.e. reflexive and transitive binary relations, while $\mathrm{Eq} A$ the set of equivalences, i.e. reflexive, transitive and symmetric binary relations.

Our use of the term "quasiorder" thus differs from the more regular usage: usually "quasiorder" and "preorder" are used interchangeably. Note that the preorder lattice is a special quasiorder lattice, and the equivalence lattice is a special congruence lattice (one can consider a set as an algebra with empty set of operations). As this work is predominantly about quasiorders and congruences, we will not specifically define the tolerance lattice and the lattice of reflexive compatible relations of algebras with an empty set of operations. (Actually, these are rather uninteresting, being lattices that are direct powers of the twoelement lattice.)

The nature of the join operation in a quasiorder lattice (which is the same as in the preorder lattice of the underlying set) is as follows:

Proposition 2.5. For $\alpha, \beta \in$ Quo A, $\alpha \vee \beta=\operatorname{Tr}(\alpha \cup \beta)$.

Again, this differs from the join in $R(\mathbf{A})$ and Tol $\mathbf{A}$. In particular, join in $R(\mathbf{A})$ and Tol $\mathbf{A}$ cannot be described without the operations of the algebra: if $\mathbf{A}$ and $\mathbf{A}^{\prime}$ are algebras with the same underlying set, $\rho$ and $\tau$ are binary relations on this set that are tolerances of both $\mathbf{A}$ and $\mathbf{A}^{\prime}$, it can happen that $\rho \vee \tau$ differs in Tol $\mathbf{A}$ and $\mathrm{Tol} \mathbf{A}^{\prime}$.

Definition 2.6. The preorders $\alpha$ and $\beta$ commute if

$$
\alpha \vee \beta=\alpha \circ \beta:=\{(a, b): \exists c: a \stackrel{\alpha}{\rightarrow} c \stackrel{\beta}{\rightarrow} b\} .
$$

This can be equivalently stated as $\alpha \circ \beta=\beta \circ \alpha$.

$R(\mathbf{A})$, Quo $\mathbf{A}$ and $\operatorname{Pre} A$ can be naturally made into involutive lattices with the involution $\delta \mapsto \delta^{-1}$, where $\delta^{-1}$ is defined by

$$
(a, b) \in \delta^{-1} \Leftrightarrow(b, a) \in \delta .
$$


While this natural involution will be used numerously, it should be noted that this thesis is only concerned for the above as lattices (this primarily matters when considering sublattices).

For any preorder $\delta$ on $A$ there correspond two equivalences: $\delta^{*}:=\delta \wedge \delta^{-1}$ and $\delta \vee \delta^{-1}$. We note that some authors use the notation $\delta^{*}$ for the latter instead of the former. There is also a poset that naturally corresponds to $\delta$ : the factor of $\delta$ by $\delta^{*}$. (This is a poset with underlying set $A / \delta^{*}$, with $(u, v) \in \delta / \delta^{*}$ iff there is $(a, b) \in \delta$ such that $a / \delta^{*}=u$ and $b / \delta^{*}=v$. While this definition can be used to factor any preorder with any equivalence, it is natural only if the equivalence is a subset of the preorder.)

\subsection{The term clone and the polynomial clone}

Definition 2.7. For natural $1 \leq i \leq n$, the $i$-th $n$-ary projection is the $n$-ary operation satisfying $e\left(x_{1}, \ldots, x_{n}\right)=x_{i}$ for all $x_{1}, \ldots, x_{n} \in A$.

We do not use any special notation for projections, for in our estimation they more often hinder than help readability.

Definition 2.8. A set $\mathcal{C}$ consisting of some operations on a set $A$ is called a clone on $A$ if it satisfies the following two properties:

- $\mathcal{C}$ contains the $i$-th $n$-ary projection on $A$ for all $1 \leq i \leq n$,

- if $f \in \mathcal{C}$ is $n$-ary, and $g_{1}, \ldots, g_{n} \in \mathcal{C}$ are all $k$-ary, then the $k$-ary operation $f\left(g_{1}, \ldots, g_{n}\right)$ is also in $\mathcal{C}$ (in other words, $\mathcal{C}$ is closed to composition).

It is obvious that the intersection of any number of clones on a given set is itself a clone. This has two immediate consequences: the first is that the clones on a set $A$ form a complete lattice (with respect to inclusion), this is denoted by Clo $A$. The second is that for any set $D$ of operations on $A$, there is a smallest clone $\mathcal{C}$ containing $D$, called the clone generated by $D$.

Definition 2.9. An operation $f$ on $A$ is idempotent if $f(a, \ldots, a)=a$ for all $a \in A$. The set of idempotent operations on $A$ is a clone, denoted by $\operatorname{Id}(A)$.

$A$ constant is a 0 -ary operation. However, an operation is constant (without article) if its value is not dependent of its input.

Definition 2.10. The clone generated by all the basic operations of an algebra $\mathbf{A}$ is called the term clone of $\mathbf{A}$, denoted by Term $\mathbf{A}$. Its elements are called terms, the set of $n$-ary terms is denoted by $\operatorname{Term}_{n} \mathbf{A}$.

The clone generated by the union of all the basic operations and the constants of an algebra $\mathbf{A}$ is called the polynomial clone of $\mathbf{A}$, denoted by Pol $\mathbf{A}$. Its elements are called polynomials, the set of $n$-ary polynomials is denoted by $\mathrm{Pol}_{n} \mathbf{A}$.

Proposition 2.11. For any $p \in \mathrm{Pol}_{k} \mathbf{A}$, there is a natural $n$, a term $t \in$ $\operatorname{Term}_{n} \mathbf{A}$, and elements $c_{k+1}, \ldots, c_{n} \in A$ such that

$$
p\left(x_{1}, \ldots, x_{k}\right)=t\left(x_{1}, \ldots, x_{k}, c_{k+1}, \ldots, c_{n}\right) .
$$


Proof. This is straightforward: the set of all $p$ satisfying the statement of the proposition obviously includes all the constants and all the terms, closed to composition, and contains only polynomials, therefore it is the set of the polynomials.

Proposition 2.12. Any relation that is compatible with an algebra is also compatible with any term of the algebra. If the relation is binary and reflexive, it is compatible with any polynomial of the algebra.

\subsection{Varieties, quasivarieties and Mal'tsev classes}

This section is based on [3] and [22].

The signature of an algebra $\mathbf{A}=\left(A,\left\{f_{i}: i \in I\right\}\right)$ is the mapping

$$
\sigma: I \mapsto \mathbf{N}_{0}, i \mapsto \operatorname{ar} f_{i}
$$

The pair $\left(i, \operatorname{ar} f_{i}\right)$ (an operation symbol with its arity) is called an abstract operation. Thus, a signature is a collection of abstract operations.

Here we need abstract clones, which are the same as regular (sometimes called operational) clones, with the exception that the notion of the $n$-ary operation is abstracted.

Definition 2.13. A set $\mathcal{C}$ consisting of some abstract operations is called an abstract clone if it satisfies the following properties:

- $\mathcal{C}$ contains an abstract operation called the $j$-th $n$-ary projection for all $1 \leq j \leq n$,

- if $f \in \mathcal{C}$ is $n$-ary, and $g_{1}, \ldots, g_{n} \in \mathcal{C}$ are all $k$-ary, then there is a $k$-ary operation $f\left(g_{1}, \ldots, g_{n}\right)$ in $\mathcal{C}$ (in other words, $\mathcal{C}$ is closed to composition),

- if $e$ is the $j$-th $n$-ary projection, and $g_{1}, \ldots, g_{n} \in \mathcal{C}$ are all $k$-ary, then $e\left(g_{1}, \ldots, g_{n}\right)=g_{j}$.

Just as for regular clones, abstract clones are closed to intersection, thus one can talk about the abstract clone generated by some abstract operations. Also, any regular clone can be considered as an abstract clone.

Definition 2.14. For each signature $\sigma$, the term clone of $\sigma$, denoted by Term $\sigma$, is the abstract clone satisfying:

- Term $\sigma$ is generated by the abstract operations $\left(i, \operatorname{ar} f_{i}\right)$ of $\sigma$,

- for any $n$-ary $f$ and any $k$-ary $g_{1}, \ldots, g_{n}$ in Term $\sigma$, either $f$ is a projection, or $f\left(g_{1}, \ldots, g_{n}\right)$ differs from each of the $g_{j}$,

- if $f$ is an $n$-ary, $f^{\prime}$ is an $n^{\prime}$-ary, $g_{1}, \ldots, g_{n}, g_{1}^{\prime}, \ldots, g_{n^{\prime}}^{\prime}$ are all $k$-ary abstract operations of Term $\sigma$, and

$$
f\left(g_{1}, \ldots, g_{n}\right)=f^{\prime}\left(g_{1}^{\prime}, \ldots, g_{n^{\prime}}^{\prime}\right),
$$

then either $f$ or $f^{\prime}$ is a projection, or $f=f^{\prime}, n=n^{\prime}$, and $g_{j}=g_{j}^{\prime}$ for all $j$. 
It is easy to see that Term $\sigma$ is well-defined. It is a free abstract clone in the following sense: if an other abstract clone $\mathcal{D}$ is generated by the same abstract operations, then the identical mapping on this common generator extends into a surjective clone homomorphism from Term $\sigma$ onto $\mathcal{D}$ (for abstract clones, a homomorphism is a mapping that maps the $i$-th $n$-ary projection for into the $i$-th $n$-ary projection for all $i$ and $n$, and preserves compositions).

If $\mathbf{A}$ is an algebra with signature $\sigma$, then the abstract operations in Term $\sigma$ naturally induce operations in Term A. Again, we will not distinguish between the operation symbol and the induced operation in notation.

Definition 2.15. Suppose $\mathbf{A}$ has signature $\sigma$, and $p, q \in$ Term $\sigma$. Then $\mathbf{A}$ is said to satisfy the identity $p \approx q$ if $p$ and $q$ induce the same operations on $\mathbf{A}$, or in other words, if $\operatorname{ar} p=\operatorname{ar} q$, and $p\left(a_{1}, \ldots, a_{\operatorname{ar} p}\right)=q\left(a_{1}, \ldots, a_{\operatorname{ar} p}\right)$ for all $a_{1}, \ldots, a_{\text {ar } p} \in A$.

The set of algebras of signature $\sigma$ is called a variety if there is a set $\Sigma$ of identities in $\sigma$ such that an algebra is in the set if and only if it satisfies all identities of $\Sigma$.

The term clone a variety $\mathcal{V}$ of signature $\sigma$, denoted by Term $\mathcal{V}$, is the factor of Term $\sigma$ by $\Sigma$, in other words, the abstract operations of Term $\mathcal{V}$ are the $\sim$-classes of Term $\sigma$, where $p \sim q$ is defined by $p \approx q \in \Sigma$, and the projections and the compositions are inherited from Term $\sigma$. The set of $k$-ary abstract operations of Term $\mathcal{V}$ is denoted by $\operatorname{Term}_{k} \mathcal{V}$.

Birkhoff's famous theorem states.

Theorem 2.16. A set of algebras of the same signature is a variety if and only if it is closed to taking subalgebras, homomorphic images, and direct products.

Definition 2.17. An algebra is finitely generated if there is a finite set of its elements so that it does not have a proper subalgebra containing all these elements.

A variety $\mathcal{V}$ is finitely generated if there is a finite algebra $\mathbf{A}$ that generates $\mathcal{V}$ (in other words, $\mathcal{V}$ is the set of algebras that satisfy any identity that $\mathbf{A}$ satisfies).

A variety is locally finite if any finitely generated algebra in it is finite.

Proposition 2.18. If a variety is finally generated, then it is locally finite.

Definition 2.19. Suppose that $p_{1}, \ldots, p_{k}, q_{1}, \ldots, q_{k}, p, q \in$ Term $\sigma$ are all $n$-ary. Then the formula

$$
\left(p_{1} \approx q_{1} \wedge \ldots \wedge p_{k} \approx q_{k}\right) \rightarrow p \approx q
$$

is called a quasi-identity. An algebra $\mathbf{A}$ of signature $\sigma$ satisfies this quasiidentity if for any $a_{1}, \ldots, a_{n} \in A$ satisfying $p_{i}\left(a_{1}, \ldots, a_{n}\right)=q_{i}\left(a_{1}, \ldots, a_{n}\right)$ for all $i, p\left(a_{1}, \ldots, a_{n}\right)=q\left(a_{1}, \ldots, a_{n}\right)$ is also satisfied.

A set of algebras is a quasivariety, if membership of it can be characterized by the satisfaction of a set of quasi-identities. 
The membership of an algebra in a variety/quasivariety depends on whether a certain fixed composition of its basic operations satisfies the needed identities. The word "fixed" is important here. For example, K-algebras (vector spaces with associative bilinear multiplication) are not Lee-algebras, because Lee-algebras satisfy the identity $a b=-b a$, while K-algebras do not (the Leebracket is written as multiplication so that it has the same signature as a $\mathrm{K}$ algebra). Nevertheless, with $a \star b:=a b-b a$, a K-algebra "becomes" a Leealgebra: any identity satisfied by Lee-algebras is also satisfied by K-algebras, one needs only to change the way of composition of the basic operations. This is the basis of the idea of Mal'tsev-classes.

Definition 2.20. Suppose that $t_{1}, \ldots, t_{n}$ are abstract operations forming the signature $\sigma, p_{1}, \ldots, p_{k}, q_{1}, \ldots, q_{k} \in \operatorname{Term} \sigma$ so that $p_{i}$ and $q_{i}$ have the same arities for each $i$. Then the strong Mal'tsev class characterized by the identities $p_{1} \approx q_{1}, \ldots, p_{k} \approx q_{k}$ is the set of algebras $\mathbf{A}$ having terms $\bar{t}_{1}, \ldots, \bar{t}_{n} \in$ Term $\mathbf{A}$ so that for all $j, \operatorname{ar} \bar{t}_{j}=\operatorname{ar} t_{j}$, and the clone homomorphism induced by $t_{j} \mapsto \bar{t}_{j}$ maps $p_{1}, \ldots, p_{k}, q_{1}, \ldots, q_{k}$ into terms $\bar{p}_{1}, \ldots, \bar{p}_{k}, \bar{q}_{1}, \ldots, \bar{q}_{k} \in$ Term A satisfying $\overline{p_{i}} \approx \overline{q_{i}}$ for all $i$.

A class of algebras $\mathfrak{K}$ is a Mal'tsev-class if there are strong Mal'tsev-classes $\mathfrak{K}_{1} \subseteq \mathfrak{K}_{2} \subseteq \ldots$ such that $\mathfrak{K}=\bigcup \mathfrak{K}_{i}$.

A variety is in a Mal'tsev-class if all of its algebras are.

For example, grupoids with commutative multiplication form a variety, while algebras having a binary commutative term form a strong Mal'tsev-class. The algebras in a Mal'tsev-class do not all have the same signature. Notice that if an algebra is in a Mal'tsev-class, then so is the variety generated by it.

The most well-known Mal'tsev-class is the original one.

Definition 2.21. A ternary operation $t$ is called a Mal'tsev-operation if it satisfies

$$
t(x, x, y) \approx t(y, x, x) \approx y \text {. }
$$

A term (polynomial) of an algebra that is a Mal'tsev-operation is called a Mal'tsev-term (Mal'tsev-polynomial).

The Mal'tsev-class associated with these identities has a very important link with congruence lattices.

Definition 2.22. An algebra is congruence permutable if any two congruences of it permute in the sense of Definition 2.6. A variety is congruence permutable if all of its algebras are.

Theorem 2.23. An algebra has a Mal'tsev-term if and only if the variety generated by it is congruence permutable.

The classical algebraic structures have a Mal'tsev-term: in a group, $x y^{-1} z$ is one, and rings, modules, fields all contain an underlying (commutative) group. Unfortunately, this excludes these structures from the scope of this thesis for the following reason: 
Proposition 2.24. If $\mathbf{A}$ has a Mal'tsev-polynomial, then $R(\mathbf{A})=\mathrm{Tol} \mathbf{A}$, and Quo $\mathbf{A}=$ Con $\mathbf{A}$.

Proof. For any $\rho \in R(\mathbf{A})$ and any $(a, b) \in \rho$,

$$
b=t(a, a, b) \stackrel{\rho}{\rightarrow} t(a, b, b)=a,
$$

as the Mal'tsev-polynomial $t$ is compatible with $\rho$ and $\rho$ is reflexive. Therefore, $\rho$ is a tolerance. The second statement is an obvious consequence of the first.

Definition 2.25. The ternary terms $t_{1}, \ldots, t_{n}$ are called Hagemann-Mitschke terms if they satisfy the following identities:

$$
\begin{aligned}
y & \approx t_{1}(x, x, y) \\
t_{i}(x, y, y) & \approx t_{i+1}(x, x, y) \text { for all } i \\
t_{n}(x, y, y) & \approx x
\end{aligned}
$$

These were introduced in [20] to characterize congruence $n+1$-permutability of a variety: the property that if $\alpha$ and $\beta$ are congruences of an algebra in the variety, then $\alpha \vee \beta=\alpha \circ \beta \circ \alpha \circ \beta \circ \ldots$, where the number of o-s on the right side is $n$. For $n=1$, this gives permutability, and the Hagemann-Mitschke-term in this case is a Mal'tsev-term.

Proposition 2.26. If $\mathbf{A}$ has Hagemann-Mitschke terms, then $\mathrm{Quo} \mathbf{A}=$ Con $\mathbf{A}$.

Proof. If $\rho \in$ Quo $\mathbf{A}$, and $(a, b) \in \rho$, then

$$
b=t_{1}(a, a, b) \stackrel{\rho}{\rightarrow} t_{1}(a, b, b)=t_{2}(a, a, b) \stackrel{\rho}{\rightarrow} \ldots \stackrel{\rho}{\rightarrow} t_{n}(a, b, b)=a .
$$

So $\rho$ is a congruence.

It is folklore that a converse of this is also true: if in a variety all quasiorders are congruences, then the variety has Hagemann-Mitscke terms.

\subsection{Semidistributivity, lower and upper bounded lattices}

Probably the most prominent lattice quasi-identities (discounting those equivalent to lattice identities, of course) are meet and join semidistributivity. The former is

$$
x \wedge y=x \wedge z \rightarrow x \wedge y=x \wedge(y \vee z),
$$

the latter is its dual. A lattice is semidistributive if it satisfies both of these quasi-identities.

The smallest-cardinality non-semidistributive lattice is $M_{3}$. The lattice $D_{1}$, and its dual, $D_{2}$, are examples for lattices only satisfying one of the semidistributive identities (the former is join, the latter is meet semidistributive). By Dedekind's condition for modularity and Birkhoff's for distributivity: 

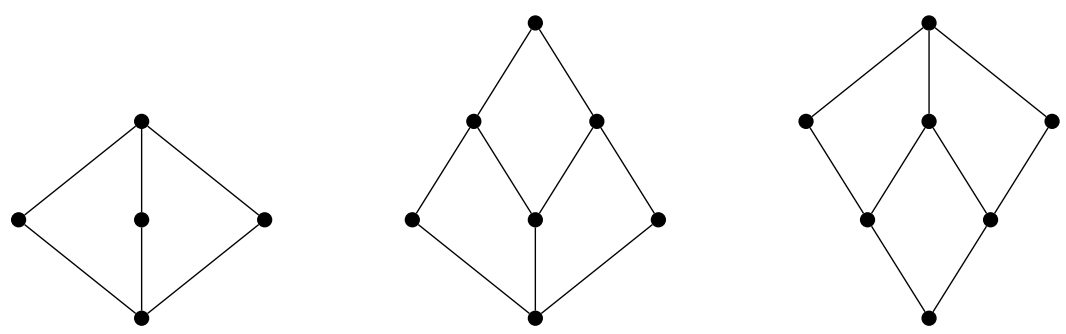

Figure 2.1: The lattices $M_{3}, D_{1}$, and $D_{2}$

Proposition 2.27. If a modular lattice is either meet or join semidistributive, then it is distributive.

Lower and upper bounded lattices were introduced by McKenzie in [25]. Day in [9] characterized them so it is decidable in polynomial time whether a finite lattice is lower bounded. Freese, Ježek, and Nation's book Free Lattices [10] contains a thorough discussion of these notions. We give here a brief summary. Note that we do not use McKenzie's original definition using free lattices.

An element $l$ of a lattice $\mathbf{L}$ is join irreducible if there are no elements $l_{1}, l_{2}<l$ such that $l_{1} \vee l_{2}=l$. It is completely join irreducible if either it is the smallest element of the lattice, or there is a largest element $l^{*}$ among all the elements of the lattice smaller then $l$. If an element is completely join irreducible, then it is also join irreducible, and for finite lattices the converse is also true. The element is join prime if for all $l_{1}, l_{2}$ satisfying $l_{1} \vee l_{2} \geq l$, either $l_{1} \geq l$ or $l_{2} \geq l$. Join primes are join irreducibles. For distributive lattices, the converse is also true.

All elements of a lattice has a $d$-rank, defined as follows. The elements with rank zero are the join primes. The elements with rank one are those elements $l$ satisfying

- $l$ is not a join prime,

- for all $a_{1}, \ldots, a_{k}$ satisfying $a_{1} \vee \cdots \vee a_{k} \geq l$ there are join prime elements $b_{1}, \ldots, b_{n}$ such that $b_{1} \vee \cdots \vee b_{n} \geq l$, and for all index $i$ there is an index $j$ with $b_{i} \leq a_{j}$.

Then recursively, the elements with rank $s$ are those elements $l$ satisfying

- $l$ does not have a rank smaller then $s$,

- for all $a_{1}, \ldots, a_{k}$ satisfying $a_{1} \vee \cdots \vee a_{k} \geq l$ there are elements $b_{1}, \ldots, b_{n}$ with rank smaller then $s$ such that $b_{1} \vee \cdots \vee b_{n} \geq l$, and for all index $i$ there is an index $j$ with $b_{i} \leq a_{j}$.

An element of a lattice that does not have a finite rank is said to have infinite rank. Denote by $d(l)$ the rank of an element $l$, by $D_{s}(\mathbf{L})$ the set of elements with rank at most $s$, and by $D(\mathbf{L})$ the set of elements of finite rank. 
Definition 2.28. A lattice is lower bounded, if it is finitely generated and satisfies $D(\mathbf{L})=L$.

The join dependency relation (also denoted by $D$ ) is a binary relation on the set of join irreducible elements of $\mathbf{L}$. It is defined by

$$
a D b \Leftrightarrow a \neq b, \exists c:(a \leq b \vee c, \forall d<b: a \not \leq d \vee c) .
$$

If $b$ is completely join irreducible, this is simplified into

$$
a D b \Leftrightarrow a \neq b, \exists c:\left(a \leq b \vee c, a \not \leq b^{*} \vee c\right) .
$$

Theorem 2.29. ([10], Theorem 2.38) If a lattice is finitely generated, then it is lower bounded if and only if the graph induced by the relation $D$ does not contain an infinite (directed) path.

Of course, for finite lattices, not containing infinite paths is the same as not containing cycles.

There is yet another characterization of lower boundedness for finite lattices.

Definition 2.30. A subset $K \subseteq L$ of the elements of the lattice $\mathbf{L}$ is called convex if for any $l_{1}, l_{2}, l_{3} \in L, l_{1}<l_{2}<l_{3}$ and $l_{1}, l_{3} \in K$ implies $l_{2} \in K$. It is called a lower pseudointerval if it is convex and has a smallest element, and an interval if it is convex and has both a smallest and a largest element.

Definition 2.31. If $\mathbf{L}$ is a lattice, and $K \subseteq L$ is convex, then the doubling of $\mathbf{L}$ by $K$, denoted by $\mathbf{L}[K]$, is the lattice with underlying set $(L \backslash K) \cup(K \times\{b, t\})$ satisfying the following conditions:

- for any $k_{1}, k_{2} \in K$,

$$
\begin{aligned}
&\left(k_{1}, b\right) \leq_{\mathbf{L}[K]}\left(k_{2}, b\right) \Leftrightarrow\left(k_{1}, b\right) \leq_{\mathbf{L}[K]}\left(k_{2}, t\right) \Leftrightarrow \\
&\left(k_{1}, t\right) \leq_{\mathbf{L}[K]}\left(k_{2}, t\right) \Leftrightarrow k_{1} \leq_{\mathbf{L}} k_{2},
\end{aligned}
$$

- for any $k_{1}, k_{2} \in K,\left(k_{1}, t\right) \not \leq\left(k_{2}, b\right)$,

- for any $k \in K$ and $l \in L \backslash K$,

$$
(k, b) \leq_{\mathbf{L}[K]} l \Leftrightarrow(k, t) \leq_{\mathbf{L}[K]} l \Leftrightarrow k \leq_{\mathbf{L}} l
$$

and

$$
(k, b) \geq_{\mathbf{L}[K]} l \Leftrightarrow(k, t) \geq_{\mathbf{L}[K]} l \Leftrightarrow k \geq_{\mathbf{L}} l,
$$

- for any $l_{1}, l_{2} \in L \backslash K, l_{1} \leq l_{2}$ is true in $\mathbf{L}[K]$ iff it is true in $\mathbf{L}$.

Theorem 2.32. ([10], Corollary 2.44) If $\mathbf{L}$ is lower bounded, and $K$ is a lower pseudointerval of it, then $\mathbf{L}[K]$ is also lower bounded. Furthermore, each finite lower bounded lattice can be obtained by the one-element lattice with a succession of doubling of lower pseudointervals. 


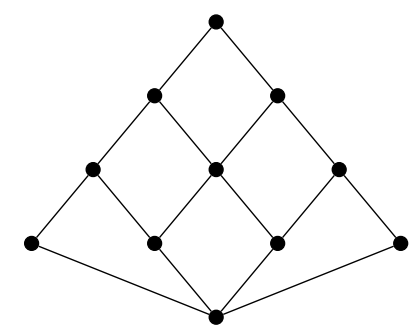

Figure 2.2: A join semidistributive, but not lower bounded lattice

A lattice is upper bounded if its dual is lower bounded, and bounded if it is both upper and lower bounded. Some authors use the term McKenzie-bounded instead, in order to avoid confusion with the other use of the term "bounded lattice", namely, a lattice with both smallest and largest element. In this thesis, a bounded lattice always means a lattice bounded in the sense of McKenzie. A lattice with both smallest (resp. largest) element will be referred to as a lattice with 0 (resp. a lattice with 1 ). Furthermore, if an element of a lattice is denoted by 0 (resp. 1), it is automatically assumed to be the smallest (resp. largest) element of that lattice. For the sake of simplicity, we denote with $0_{\mathbf{B}}$ (resp. $\left.1_{\mathbf{B}}\right)$ the equality (resp. the full) relation on the underlying set of the algebra $\mathbf{B}$ instead of $0_{\mathrm{Con} B}$ or $0_{\mathrm{Quo}} \mathrm{B}$.

Theorem 2.33. ([10], Corollary 2.17) The class of finite lower (upper) bounded lattices is closed to taking sublattices, homomorphic images, and finite direct products.

Theorem 2.34. ([10], Theorem 2.20) All lower (upper) bounded lattices are join (meet) semidistributive.

The converse is not true, as Figure 2.2 shows. 


\section{Chapter 3}

\section{Lattice representations with DCC posets}

Probably the most basic representation theorem of lattice theory is Whitman's result stating that every lattice is embeddable into the lattice of equivalences of a large enough set [31]. A complementary result is proved by Achein in [1]:

Theorem 3.1. For any lattice $\mathbf{L}$ there is a set $X$ and an injective homomorphism $\gamma: L \mapsto \operatorname{Pre} X$ so that the image of this homomorphism contains only posets. Moreover, $X$ and $\gamma$ can be chosen so that the elements of $\gamma(L)$ pairwise commute.

This embeddability will be referred to as " $\mathbf{L}$ is representable as a lattice of posets," or more shortly, "L is representable with posets". (The posets on a given set do not form a lattice. Under a lattice of posets we mean a sublattice of a preorder lattice containing only antisymmetric preorders.)

For finite lattices, the equivalance representation can be given on an underlying finite set [28]. This is not true for the poset representation. Sivak in [30] gave a characterization for lattices representable as lattices of posets on a finite set using the notion of small congruences of a lattice (a kind of congruences where every congruence class has at most two elements). In [2], the authors note that this characterization precisely describe the class of finite lower bounded lattices.

Theorem 3.2. A finite lattice is representable with posets on a finite underlying set iff it is lower bounded.

This can be seen as a generalization of a theorem of Caspard [4], namely that the lattice of permutations of a finite set is (both lower and upper) bounded. Here the lattice of permutations means the lattice induced by the following order: we fix a linear order on the underlying set, and a permutation $\pi_{1}$ will be smaller then the (distinct) permutation $\pi_{2}$ iff for every pair $i<j$ of the underlying set, $\pi_{1}(i)>\pi_{1}(j)$ implies $\pi_{2}(i)>\pi_{2}(j)$. The result about lattices of posets is a generalization of this because the lattice of permutations can be seen 
as dually isomorphic to a lattice of posets: for every permutation $\pi$, take the poset $\leq_{\pi}$ defined by $i \leq_{\pi} j$ iff either $i=j$, or $i<j$ and $\pi(i)<\pi(j)$. We leave it to the reader to check that $\pi \mapsto \leq_{\pi}$ indeed defines an injective dual lattice homomorphism.

Semenova in [29] proves something more general (see Proposition 1.6. and Theorem 4.6.): any finite lattice embeddable into the suborder lattice of a poset containing no infinite chain must be lower bounded, and all lower bounded lattices are embeddable into such a lattice. Being embeddable into a suborder lattice of a poset containing no infinite chain means (at least for lattices with a largest element) of being representable with posets satisfying both the ascending and descending chain conditions. (A poset satisfies DCC and ACC if and only if it has no infinite chain.)

This chapter is concerned by the following question: what if only the DCC is required of the posets? As it is a weaker condition, all lower bounded lattices are representable in such a way, but even among finite lattices, there are representable ones that are not lower bounded.

We give an algorithmic characterization of representable finite lattices, and a general necessary condition. The latter is a kind of weaker version of lower boundedness: instead of D-cycles, it forbids cycles of D-cycles. The proofs can be adapted for Semenova's aforementioned results.

\subsection{Representation of finite lattices}

The problem of whether a finite lattice is representable with DCC posets (i.e. is isomorphic to a lattice of DCC-posets) is decidable, as the following theorem shows.

Definition 3.3. For a finite lattice $\mathbf{L}, \mathcal{C}_{\mathbf{L}}$ denotes the set of nontrivial join covers of join irreducibles, i.e. the set

$$
\begin{aligned}
& \left\{\left(l, l_{1}, \ldots, l_{k}\right) \in J(L)^{k+1}: l \leq l_{1} \vee l_{2} \vee \cdots \vee l_{k},\right. \\
& \left.l \not \leq\left(l_{1}\right)^{*} \vee l_{2} \vee \cdots \vee l_{k}, l \not \leq l_{1} \vee\left(l_{2}\right)^{*} \vee \cdots \vee l_{k}, \ldots, l \not \leq l_{1} \vee l_{2} \vee \cdots \vee\left(l_{k}\right)^{*}\right\}
\end{aligned}
$$

Theorem 3.4. A finite lattice $\mathbf{L}$ is representable with DCC posets if and only if there is a mapping $s: \mathcal{C}_{\mathbf{L}} \mapsto L$ satisfying the following:

- for any $\left(l, l_{1}, \ldots, l_{k}\right) \in \mathcal{C}_{\mathbf{L}}, s\left(l, l_{1}, \ldots, l_{k}\right) \in\left\{l_{1}, \ldots, l_{k}\right\}$,

- $s$ is symmetrical in all but the first variable, i.e. for any permutation $\pi \in S_{k}$,

$$
s\left(l, l_{1}, \ldots, l_{k}\right)=s\left(l, l_{\pi(1)}, \ldots, l_{\pi(k)}\right),
$$

- for the binary relations

$$
T_{\mathbf{L}}:=\left\{\left(l, l_{i}\right):\left(l, l_{1}, \ldots, l_{k}\right) \in \mathcal{C}_{\mathbf{L}}, s\left(l, l_{1}, \ldots, l_{k}\right) \neq l_{i}\right\}
$$


and

$U_{\mathbf{L}}:=\operatorname{Tr}\left(\{(l, l): l \in L\} \cup\left\{\left(l, l_{i}\right):\left(l, l_{1}, \ldots, l_{k}\right) \in \mathcal{C}_{\mathbf{L}}, s\left(l, l_{1}, \ldots, l_{k}\right)=l_{i}\right\}\right)$,

the relation $U_{\mathbf{L}} \circ T_{\mathbf{L}}$ does not contain a circle.

Proof. Firstly, suppose that there is such a mapping $s$.

We will give an algorithm that will yield a set $X$, partial orders $\gamma_{l} \subseteq X^{2}$ for all $l \in L$ such that the mapping $l \mapsto \gamma_{l}$ is a lattice homomorphism from $\mathbf{L}$ into Pre $X$, and all $\gamma_{l}$ satisfy DCC.

Begin with a set $X^{(0)}=\left\{x_{l, 1}: l \in L\right\} \cup\left\{x_{l, 2}: l \in L\right\}$, and for any $l \in J(L)$ define $\gamma_{l}^{(0)}$ as a binary relation on $X^{(0)}$ whose only edge is $\left(x_{l, 1}, x_{l, 2}\right)$.

Now we recursively define for all natural $j$ and $l \in J(L)$ the sets $X^{(j)}$ and binary relations $\gamma_{l}^{(j)}$ on $X^{(j)}$. Firstly, $X^{(j)}$ will be a set containing $X^{(j-1)}$, and $\gamma_{l}^{(j)}$ a relation containing $\gamma_{l}^{(j-1)}$. Secondly, for any $\left(l, l_{1}, \ldots, l_{k}\right) \in \mathcal{C}_{\mathbf{L}}$ and any $\left(y_{1}, y_{2}\right) \in \gamma_{l}^{(j-1)} \backslash\left(\gamma_{l_{1}}^{(j-1)} \vee \cdots \vee \gamma_{l_{k}}^{(j-1)}\right)$, add the elements $x_{y_{1}, y_{2}, l, l_{1}, \ldots, l_{k}, 1}, \ldots$, $x_{y_{1}, y_{2}, l, l_{1}, \ldots, l_{k}, k-1}$ to $X^{(j)}$. Use the notion $x_{y_{1}, y_{2}, l, l_{1}, \ldots, l_{k}, 0}=y_{1}, x_{y_{1}, y_{2}, l, l_{1}, \ldots, l_{k}, k}=$ $y_{2}$. For $0 \leq r<k$, add the edge $\left(x_{y_{1}, y_{2}, l, l_{1}, \ldots, l_{k}, r}, x_{y_{1}, y_{2}, l, l_{1}, \ldots, l_{k}, r+1}\right)$ into one of the $\gamma_{l_{i}}^{(j)}$ in a way that exactly one edge goes into each of the $\gamma_{l_{i}}^{(j)}$, and the last edge $\left(\left(x_{y_{1}, y_{2}, l, l_{1}, \ldots, l_{k}, k-1}, y_{2}\right)\right)$ goes into $\gamma_{s\left(l, l_{1}, \ldots, l_{k}\right)}^{(j)}$.

Finally, set $X=\bigcup X^{(j)}$ and for any $l \in L$ set

$$
\gamma_{l}=\operatorname{Tr}\left(\{(x, x): x \in X\} \cup \bigcup\left\{\gamma_{l^{\prime}}^{(j)}: j \in \mathbf{N}, l^{\prime} \in J(L), l^{\prime} \leq l\right\}\right) .
$$

It is easy to see that all $\gamma_{l}$ are partial orders on $X$, and that $\gamma_{l_{1}}<\gamma_{l_{2}}$ iff $l_{1}<l_{2}$. (The inequality is strict because there is an $l^{\prime} \in J(L)$ such that $l^{\prime} \leq l_{2}$ and $l^{\prime} \not \leq l_{1}$, and then $\left(x_{l^{\prime}, 1}, x_{l^{\prime}, 2}\right)$ is in $\gamma_{l_{2}} \backslash \gamma_{l_{1}}$.)

We will prove that $l \mapsto \gamma_{l}$ preserves meets and joins. For the "join" part, as $l \mapsto \gamma_{l}$ is order-preserving, it is enough to show that $\gamma_{l_{1} \vee l_{2}} \leq \gamma_{l_{1}} \vee \gamma_{l_{2}}$ and to do that, it is enough to show that for all $l^{\prime} \in J(L)$ with $l^{\prime} \leq l_{1} \vee l_{2}$, and all $j$, $\gamma_{l^{\prime}}^{(j)} \subseteq \gamma_{l_{1}} \vee \gamma_{l_{2}}$.

$\mathbf{L}$ is a finite lattice, so all its elements is a join of join irreducible elements: $l_{1}=p_{1} \vee \cdots \vee p_{m}$, and $l_{2}=q_{1} \vee \cdots \vee q_{n}$. Now as $l^{\prime} \leq l_{1} \vee l_{2}=p_{1} \vee \ldots p_{m} \vee q_{1} \vee$ $\cdots \vee q_{n}$

$$
\gamma_{l^{\prime}}^{(j)} \subseteq \operatorname{Tr}\left(\gamma_{p_{1}}^{(j+1)} \cup \cdots \cup \gamma_{p_{m}}^{(j+1)} \cup \gamma_{q_{1}}^{(j+1)} \cup \cdots \cup \gamma_{q_{n}}^{(j+1)}\right) \subseteq \gamma_{l_{1}} \vee \gamma_{l_{2}}
$$

For the "meet" part, take a (non-loop) edge $\left(x_{1}, x_{2}\right) \in \gamma_{l_{1}} \wedge \gamma_{l_{2}}$. If both $x_{1}$ and $x_{2}$ are in $X^{(0)}$, there is an $l^{\prime} \in J(L)$ such that $\left(x_{1}, x_{2}\right)=\left(x_{l^{\prime}, 1}, x_{l^{\prime}, 2}\right)$. Therefore, $l^{\prime} \leq l_{1}$ and $l^{\prime} \leq l_{2}$, so $l^{\prime} \leq l_{1} \wedge l_{2}$, and $\left(x_{1}, x_{2}\right) \in \gamma_{l_{1} \wedge l_{2}}$.

Otherwise, it can be assumed that both $x_{1}$ and $x_{2}$ are in $X^{(j)}$, but $x_{1}$ is not in $X^{(j-1)}$, so $x_{1}=x_{y_{1}, y_{2}, l^{\prime}, l_{1}^{\prime}, \ldots, l_{k}^{\prime}, r}$, where $y_{1}, y_{2} \in X^{(j-1)}, l^{\prime} \leq l_{1}^{\prime} \vee \cdots \vee l_{k}^{\prime}$, and $\left(y_{1}, y_{2}\right) \in \gamma_{l^{\prime}}^{(j-1)} \backslash\left(\gamma_{l_{1}^{\prime}}^{(j-1)} \vee \cdots \vee \gamma_{l_{k}^{\prime}}^{(j-1)}\right)$. Suppose that $x_{2}=x_{y_{1}, y_{2}, l^{\prime}, l_{1}^{\prime}, \ldots, l_{k}^{\prime}, r^{\prime}}$ for some $r<r^{\prime}$, in which case $\left(x_{1}, x_{2}\right) \in \gamma_{l_{1}} \wedge \gamma_{l_{2}}$ is only possible if

$$
\begin{array}{r}
\left(x_{y_{1}, y_{2}, l^{\prime}, l_{1}^{\prime}, \ldots, l_{k}^{\prime}, r}, x_{y_{1}, y_{2}, l^{\prime}, l_{1}^{\prime}, \ldots, l_{k}^{\prime}, r+1}\right), \ldots,\left(x_{y_{1}, y_{2}, l^{\prime}, l_{1}^{\prime}, \ldots, l_{k}^{\prime}, r^{\prime}-1}, x_{y_{1}, y_{2}, l^{\prime}, l_{1}^{\prime}, \ldots, l_{k}^{\prime}, r^{\prime}}\right) \\
\in \gamma_{l_{1}} \wedge \gamma_{l_{2}},
\end{array}
$$


because any $\gamma_{l}$ on $X^{(j)}$ coincides with the reflexive and transitive closure of $\gamma_{l}^{(j)}$. Hence, $l_{1}, l_{2} \leq l_{r}^{\prime}, \ldots, l_{r^{\prime}-1}^{\prime}$, and thus $l_{1} \wedge l_{2} \leq l_{r}^{\prime}, \ldots, l_{r^{\prime}-1}^{\prime}$, and $\left(x_{1}, x_{2}\right) \in \gamma_{l_{1} \wedge l_{2}}$.

If $x_{2}$ is not of the form $x_{y_{1}, y_{2}, l^{\prime}, l_{1}^{\prime}, \ldots, l_{k}^{\prime}, r^{\prime}}$, then (again because $\gamma_{l}$ on $X^{(j)}$ coincides with the reflexive and transitive closure of $\left.\gamma_{l}^{(j)}\right)\left(x_{1}, x_{2}\right) \in \gamma_{l_{1}} \wedge \gamma_{l_{2}}$ is only possible if $\left(x_{1}, y_{2}\right)$ and $\left(y_{2}, x_{2}\right)$ are in both in $\gamma_{l_{1}} \wedge \gamma_{l_{2}}$. As $y_{2}=x_{y_{1}, y_{2}, l^{\prime}, l_{1}^{\prime}, \ldots, l_{k}^{\prime}, k}$, by the previous argument, $\left(x_{1}, y_{2}\right) \in \gamma_{l_{1} \wedge l_{2}}$.

We still need that $\left(y_{2}, x_{2}\right) \in \gamma_{l_{1} \wedge l_{2}}$. If $x_{2}$ is in $X^{(j-1)}$, then we are done by induction. Otherwise, by repeating to $\left(y_{2}, x_{2}\right)$ what we did to $\left(x_{1}, x_{2}\right)$, we will get an $y_{1}^{\prime} \in X^{(j-1)}$ such that it is enough to prove $\left(y_{2}, y_{1}^{\prime}\right) \in \gamma_{l_{1} \wedge l_{2}}$ to prove $\left(y_{2}, x_{2}\right) \in \gamma_{l_{1} \wedge l_{2}}$. That, again, can be done by induction.

The only thing left to prove is that $\gamma_{1}$ satisfies DCC ( 1 denoting the largest element of $\mathbf{L}$ ).

For any $l \in L$, the difficulty of $l$ will denote the length of the longest $U_{\mathbf{L}} \circ T_{\mathbf{L}^{-}}$ path starting from $l$. This is finite because $\mathbf{L}$ is a finite lattice, and $U_{\mathbf{L}} \circ T_{\mathbf{L}}$ contains no circle.

An edge of the type $\left(x_{l, 1}, x_{l, 2}\right)$ will be called an original edge, an edge of the type

$$
\left(x_{y_{1}, y_{2}, l, l_{1}, \ldots, l_{k}, r}, x_{y_{1}, y_{2}, l, l_{1}, \ldots, l_{k}, r+1}\right)
$$

with $r<k-1$ a lower part, an edge $\left(x_{y_{1}, y_{2}, l, l_{1}, \ldots, l_{k}, k-1}, y_{2}\right)$ an upper part of the edge $\left(y_{1}, y_{2}\right)$. An edge is a core edge if it either original, or a lower or upper part of a (core) edge. Thus, all $\gamma_{l}$ is the reflexive and transitive closure of the set of the core $\gamma_{l}$ edges. An edge is an upper edge if it of the form $\left(x_{y_{1}, y_{2}, l, l_{1}, \ldots, l_{k}, h}, y_{2}\right)$ for some $0<h<k$.

Suppose $\left(a_{i}\right)_{i \in \mathbf{N}}$ is an infinite (strictly) decreasing sequence in $\gamma_{1}$. It can be assumed that all the $\left(a_{i+1}, a_{i}\right)$ are core edges (otherwise, it is the concatenation of core edges, which can be split to core edges). Now after any upper core edge $\left(x_{y_{1}, y_{2}, l, l_{1}, \ldots, l_{k}, k-1}, y_{2}\right)$ in the sequence, the following edge is either an other upper core one, or it is the lower core edge

$$
\left(x_{y_{1}, y_{2}, l, l_{1}, \ldots, l_{k}, k-2}, x_{y_{1}, y_{2}, l, l_{1}, \ldots, l_{k}, k-1}\right),
$$

after which the only possible lower core one is

$$
\left(x_{y_{1}, y_{2}, l, l_{1}, \ldots, l_{k}, k-3}, x_{y_{1}, y_{2}, l, l_{1}, \ldots, l_{k}, k-2}\right),
$$

and so on. It can be assumed that there is an upper core edge before reaching $\left(y_{1}, x_{y_{1}, y_{2}, l, l_{1}, \ldots, l_{k}, 1}\right)$, because otherwise one can exchange all these edges to their concatenation $\left(y_{1}, y_{2}\right)$, which is still a core edge. Thus, by concatenating any upper part edge with all the lower part ones following it before the next upper part, one gets a sequence of $\gamma_{1}$-edges $\left(b_{i+1}, b_{i}\right)_{i \in \mathbf{N}}$ such that all of those edges are upper edges (though not necessarily cores).

The following claim presents an immediate contradiction to the existance of such $\left(b_{i}\right)_{i \in \mathbf{N}}$.

Claim 1. For any $b=x_{y_{1}, y_{2}, l, l_{1}, \ldots, l_{k}, r}$ and any path of upper part edges ending in $b$, this path has length not greater than the difficulty of the $l_{j}$ satisfying $\left(x_{y_{1}, y_{2}, l, l_{1}, \ldots, l_{k}, r-1}, b\right) \in \gamma_{l_{j}}$. 
Let $d=x_{y_{1}, y_{2}, l, l_{1}, \ldots, l_{k}, r-1}$.

The claim is proved by induction on the difficulty of $l_{j}$. Suppose first that it is 0 . This means that there is no $T_{\mathbf{L}}$-edge with source $l_{j}$, consequently, $l_{j}$ is a join prime, and there is no element of the form $x_{d, b, h, h_{1}, \ldots, h_{t}, q}$, and no upper edge ending in $b$.

Now suppose that the difficulty of $l_{j}$ is positive. Let $(c, b)$ be an upper

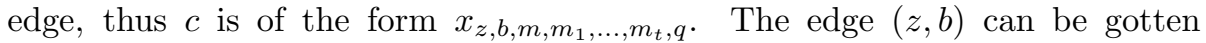
by repeatedly taking upper parts of the edge $\left(y_{1}, b\right)$, therefore $\left(l_{j}, m\right) \in U_{\mathbf{L}}$. Suppose that $\left(x_{z, b, m, m_{1}, \ldots, m_{t}, q-1}, c\right)$, which is a lower part of the edge $(z, b)$, is in $\gamma_{m_{j^{\prime}}}$, then $\left(m, m_{j^{\prime}}\right) \in T_{\mathbf{L}}$. Thus $\left(l_{j}, m_{j^{\prime}}\right) \in U_{\mathbf{L}} \circ T_{\mathbf{L}}$, so the difficulty of $m_{j^{\prime}}$ is smaller than the difficulty of $l_{j}$. By the inductive hypothesis, any path of upper part core edges ending in $c$ has length smaller than the difficulty of $l_{j}$. The claim is proved.

For the converse direction, assume that $l \mapsto \gamma_{l}$ is a lattice embedding from L into Pre $X$. For any edge $\left(z_{1}, z_{2}\right) \in \gamma_{1}$ there is a smallest $k \in L$ such that $\left(z_{1}, z_{2}\right) \in \gamma_{k}$, the weight of this edge.

An edge $(c, d)$ is called contained in the edge $(a, b)$ if both $(a, c)$ and $(d, b)$ are in $\gamma_{1}$, but $(c, d) \neq(a, b)$. It is properly contained if moreover $d<b$.

Let $\left(l, l_{1}, \ldots, l_{k}\right) \in \mathcal{C}_{\mathbf{L}}$, and take an edge $\left(a_{1}, a_{2}\right) \in \gamma_{l} \backslash \gamma_{l^{*}}$, this edge has weight $l$. There are elements $a_{1}=b_{0}, b_{1}, \ldots, b_{r}=a_{2}$ in $X$ such that for all $0 \leq i<r,\left(b_{i}, b_{i+1}\right) \in \gamma_{l_{1}} \cup \cdots \cup \gamma_{l_{k}}$. Among these edges there must be at least one with weight $l_{j}$ for all $1 \leq j \leq l$, otherwise

$$
\left(a_{1}, a_{2}\right) \in \gamma_{l} \cap\left(\gamma_{l_{1}} \vee \cdots \vee\left(\gamma_{l_{j}}\right)^{*} \vee \cdots \vee \gamma_{l_{k}}\right) \subseteq \gamma_{l^{*}} .
$$

So each edge with weight $l$ contains at least one edge of weight $l_{1}$, at least one of weight $l_{2}$, e.c., and it contains these edges properly with at most one exception.

Now define the mapping $s$ on $\left(l, l_{1}, \ldots, l_{k}\right)$ so that if there are infinitely many edges with weight $l$, but only finitely many contains an edge of weight $l_{j}$ properly, then $s\left(l, l_{1}, \ldots, l_{k}\right)=l_{j}$. If there is no such $j$, then set $s\left(l, l_{1}, \ldots, l_{k}\right)=l_{1}$.

If $\left(l, l^{\prime}\right) \in U_{\mathbf{L}} \circ T_{\mathbf{L}}$, then all edges of weight $l$ must contain an edge of weight $l^{\prime}$. Suppose there is a circle of $U_{\mathbf{L}} \circ T_{\mathbf{L}}$ containing the edge $\left(l, l^{\prime}\right)$. There is an $m \in L$ so that $(l, m) \in U_{\mathbf{L}}$ and $\left(m, l^{\prime}\right) \in T_{\mathbf{L}}$. All edges of weight $l$ must contain an edge of weight $l$. Starting from an edge $\left(f_{1}, e_{1}\right)$ of weight $l$ one can get the edges $\left(f_{2}, e_{2}\right),\left(f_{3}, e_{3}\right)$ e.c., each contained in the previous, and each having weight $l$. Furthermore, for each $j>0$ either $\left(f_{j}, e_{j}\right)$ can be chosen so that $e_{j} \neq e_{j-1}$, or it can only be chosen so that there is an edge of weight $m$ containing $\left(f_{j}, e_{j}\right)$ and contained in $\left(f_{j-1}, e_{j-1}\right)$ that does not properly contain any edge of weight $l^{\prime}$. There are only finitely many such edges of weight $m$. Therefore, the $\left(f_{j}, e_{j}\right)$ edges can be chosen so that the sequence $\left(e_{j}\right)_{j \in \mathbf{N}}$ contains an infinite strictly decreasing subsequence in $\gamma_{1}$, a contradiction.

Theorem 3.4 gives an algorithm deciding whether $\mathbf{L}$ is representable with DCC-posets. The algorithm is in $\mathcal{E} \mathcal{X} \mathcal{P} \mathcal{T} \mathcal{I M E}$.

Problem 3.5. Are there real numbers $k$ and $\alpha$ such that for all finite lattice $\mathbf{L},\left|\mathcal{C}_{\mathbf{L}}\right|<k|L|^{\alpha}$ ? 
If the answer to this is "yes", then the algorithm is actually in $\mathcal{N} \mathcal{P}$.

Conjecture 3.6. Deciding whether a finite lattice is representable with DCCposets is an $\mathcal{N} \mathcal{P}$-hard problem.

For comparison, deciding whether a finite lattice is lower bounded is in $\mathcal{P}$. (By Theorem 3.2, these are the lattices representable with posets on a finite set. We note that an adaptation of the proof of Theorem 3.4 can be used to prove that statement. One needs to use the D-rank characterization of lower boundedness.)

\subsection{Representation of arbitrary lattices}

For a lattice $\mathbf{L}$, denote with $\mathcal{C} \mathcal{Y}_{\mathbf{L}}$ the set of D-cycles of $\mathbf{L}$ consisting of completely join irreducible elements. Introduce a binary relation on $\mathcal{C} \mathcal{Y}_{\mathbf{L}}$ :

$$
\begin{aligned}
E_{\mathbf{L}}:=\left\{\left(\left(\beta_{1}, \ldots, \beta_{l}\right),\left(\alpha_{1}, \ldots, \alpha_{k}\right)\right):\right. & \\
\exists i: \exists j: \alpha_{j+1} \leq \beta_{i} \vee \alpha_{j}, \alpha_{j+1} \not \leq \beta_{i} \vee \alpha_{j}^{*}, \alpha_{j+1} & \left.\leq \beta_{i}^{*} \vee \alpha_{j}\right\},
\end{aligned}
$$

with the index $j$ meant as modulo $k$ and the index $i$ as modulo $l$.

The following is a necessary condition for a lattice to be representable with DCC posets.

Theorem 3.7. If $\mathbf{L}$ is representable with DCC-posets, then $E_{\mathbf{L}}$ does not contain a cycle.

Proof. Suppose the contrary, that

$$
\underline{\alpha}^{(1)} E_{\mathbf{L}} \underline{\alpha}^{(2)} E_{\mathbf{L}} \ldots E_{\mathbf{L}} \underline{\alpha}^{(t)} E_{\mathbf{L}} \underline{\alpha}^{(1)},
$$

with $\underline{\alpha}^{(i)}=\left(\alpha_{1}^{(i)}, \ldots, \alpha_{k_{i}}^{(i)}\right)$ for all $1 \leq i \leq t$. For all $1 \leq i \leq t$ and $1 \leq j \leq k_{i}$, there is a $\gamma_{j}^{(i)}$ that $\alpha_{j}^{(i)} \leq \gamma_{j}^{(i)} \vee \alpha_{j+1}^{(i)}$ and $\alpha_{j}^{(i)} \not \leq \gamma_{j}^{(i)} \vee\left(\alpha_{j+1}^{(i)}\right)^{*}$, with the index $j$ taken modulo $k_{i}$. By the definition of $E_{\mathbf{L}}$, it may be assumed that for all $i$ there is an index $m_{i}$ such that $\gamma_{m_{i}}^{(i)}=\alpha_{1}^{(i+1)}$ for all $1 \leq i \leq t$ (taken modulo $t$ ), and furthermore, $\alpha_{m_{i}}^{(i)} \not \leq\left(\alpha_{1}^{(i+1)}\right)^{*} \vee \alpha_{m_{i}+1}^{(i)}$ is satisfied. Set $\mu$ as the join of all the $\alpha_{j}^{(i)}$.

Let the underlying set of the posets be $X$. Start with the inequality

$$
\begin{gathered}
\alpha_{m_{1}}^{(1)} \leq \gamma_{m_{1}}^{(1)} \vee\left(\alpha_{m_{1}+1}^{(1)} \wedge\left(\gamma_{m_{1}+1}^{(1)} \vee\left(\alpha_{m_{1}+2}^{(1)} \wedge \cdots \vee\left(\alpha_{m_{1}-1}^{(1)} \wedge\left(\gamma_{m_{1}-1}^{(1)} \vee \alpha_{m_{1}}^{(1)}\right)\right)\right)\right)=\right. \\
\alpha_{1}^{(2)} \vee\left(\alpha_{m_{1}+1}^{(1)} \wedge\left(\gamma_{m_{1}+1}^{(1)} \vee\left(\alpha_{m_{1}+2}^{(1)} \wedge \cdots \vee\left(\alpha_{m_{1}-1}^{(1)} \wedge\left(\gamma_{m_{1}-1}^{(1)} \vee \alpha_{m_{1}}^{(1)}\right)\right)\right)\right)=\right. \\
\left(\alpha_{1}^{(2)} \wedge\left(\gamma_{1}^{(2)} \vee\left(\alpha_{2}^{(2)} \wedge \cdots \vee\left(\alpha_{m_{2}-1}^{(2)} \wedge\left(\gamma_{m_{2}-1}^{(2)} \vee \alpha_{m_{2}}^{(2)}\right)\right)\right)\right)\right) \vee \\
\left(\alpha_{m_{1}+1}^{(1)} \wedge\left(\gamma_{m_{1}+1}^{(1)} \vee\left(\alpha_{m_{1}+2}^{(1)} \wedge \cdots \vee\left(\alpha_{m_{1}-1}^{(1)} \wedge\left(\gamma_{m_{1}-1}^{(1)} \vee \alpha_{m_{1}}^{(1)}\right)\right)\right)\right) .\right.
\end{gathered}
$$

We will call a sequence of $L$-elements $a=c_{0}, \ldots, c_{r}=b$ a realization of the $\alpha_{m_{1}}^{(1)}$ edge $(a, b)$ if the following are satisfied: 
- For all $1 \leq s \leq r,\left(c_{s}, c_{s+1}\right)$ is an edge of either $\alpha_{m_{1}}^{(1)}$, or $\alpha_{m_{2}}^{(2)}$, or $\gamma_{j}^{(2)}$ for an $1 \leq j<m_{2}$, or $\gamma_{j}^{(1)}$ for an $1 \leq j<k_{1}, j \neq m_{1}$.

- There are indexes $0=r_{0}<r_{1}<\cdots<r_{h}=r$ such that

- For any even $0 \leq h^{\prime}<h,\left(c_{r_{h^{\prime}}}, c_{r_{h^{\prime}+1}}\right) \in \alpha_{1}^{(2)}$, and for any odd $0 \leq h^{\prime}<h,\left(c_{r_{h^{\prime}}}, c_{r_{h^{\prime}+1}}\right) \in \alpha_{m_{1}+1}^{(1)}$,

- For even $h^{\prime}$, if $r_{h^{\prime}} \leq s_{1}<s_{2} \leq r_{h^{\prime}+1}$ and $i_{0}$ are such that the set

$$
\left\{s:\left(\left(c_{s}, c_{s+1}\right) \in \alpha_{m_{2}}^{(2)} \vee \exists i: i_{0} \leq i<m_{2},\left(c_{s}, c_{s+1}\right) \in \gamma_{i}^{(2)}\right)\right\}
$$

contains all $s^{\prime}$ satisfying $s_{1} \leq s^{\prime}<s_{2}$ but does not contain $s_{1}-1$ and $s_{2}$, then $\left(c_{s_{1}}, c_{s_{2}}\right) \in \alpha_{i_{0}}^{(2)}$,

- For odd $h^{\prime}$, if $r_{h^{\prime}} \leq s_{1}<s_{2} \leq r_{h^{\prime}+1}$ and $i_{0}$ are such that the set

$$
\begin{aligned}
&\left\{s:\left(\left(c_{s}, c_{s+1}\right)\right.\right. \in \alpha_{m_{1}}^{(1)} \vee \\
&\left.\left.\exists i: i \in\left\{i_{0}, i_{0}+1, \ldots, m_{1}-1\right\},\left(c_{s}, c_{s+1}\right) \in \gamma_{i}^{(2)}\right)\right\}
\end{aligned}
$$

contains all $s^{\prime}$ satisfying $s_{1} \leq j^{\prime}<s_{2}$ but does not contain $s_{1}-1$ and $s_{2}$, then $\left(c_{s_{1}}, c_{s_{2}}\right) \in \alpha_{i_{0}}$.

Note the connection between the definition of a realization and the inequality preceding it. For each $\alpha_{m_{1}}^{(1)}$ edge we choose a single realization to get a canonical realization. We likewise obtain canonical realizations for $\alpha_{m_{i}}^{(i)}$ edges for all $1 \leq$ $i \leq t$ (just switch the lower indexes everywhere in the definition cyclically by $i-1)$. If an edge is at the same time an $\alpha_{m_{i_{1}}}^{\left(i_{1}\right)}$ and an $\alpha_{m_{i_{2}}}^{\left(i_{2}\right)}$ edge, it will get a canonical realization both as an $\alpha_{m_{i_{1}}}^{\left(i_{1}\right)}$ and as an $\alpha_{m_{i_{2}}}^{\left(i_{2}\right)}$ edge.

For an edge $\left(x_{1}, x_{2}\right) \in \alpha_{m_{i}}^{(i)}$, denote with $\mathcal{R}_{\left(x_{1}, x_{2}\right)}^{(i)}$ the set of the edges of the canonical realization of $\left(x_{1}, x_{2}\right)$ as an $\alpha_{m_{i}}^{(i)}$ edge, and set

$$
\mathcal{K}_{\left(x_{1}, x_{2}\right)}^{(i)}=\left\{\left(y_{1}, y_{2}\right) \in \mathcal{R}_{\left(x_{1}, x_{2}\right)}^{(i)}: y_{2} \neq x_{2},\left(y_{1}, y_{2}\right) \in \alpha_{m_{i}}^{(i)} \cup \alpha_{m_{i+1}}^{(i+1)}\right\} .
$$

Define a rank of the edge $\left(x_{1}, x_{2}\right)$ : it will be zero if $\mathcal{K}_{\left(x_{1}, x_{2}\right)}^{(i)}=\emptyset$, otherwise, the rank is recursively defined as the maximal rank of the elements of $\mathcal{K}_{\left(x_{1}, x_{2}\right)}^{(i)}$ plus one. As $\mu$ satisfies DCC, all $\alpha_{m_{i}}^{(i)}$ edge has a (finite) rank.

Take an $\alpha_{m_{1}}^{(1)}$ edge $\left(x_{1}, x_{2}\right)$ of rank 0 . In its canonical realization there is at most one edge that is also an $\alpha_{m_{1}}^{(1)}$ or an $\alpha_{m_{2}}^{(2)}$ edge (the last edge of the 
realization). This means that

$$
\begin{aligned}
&\left(x_{1}, x_{2}\right) \in \eta_{0}^{(1)}:= \\
&\left(\alpha_{m_{1}}^{(1)} \wedge\right.\left(\left(\alpha_{1}^{(2)} \wedge\left(\gamma_{1}^{(2)} \vee\left(\alpha_{2}^{(2)} \wedge \cdots \vee\left(\alpha_{m_{2}-1}^{(2)} \wedge \gamma_{m_{2}-1}^{(2)}\right)\right)\right)\right) \vee\right. \\
&\left.\left.\left(\alpha_{m_{1}+1}^{(1)} \wedge\left(\gamma_{m_{1}+1}^{(1)} \vee\left(\alpha_{m_{1}+2}^{(1)} \wedge \cdots \vee\left(\alpha_{m_{1}-1}^{(1)} \wedge\left(\gamma_{m_{1}-1}^{(1)} \vee \alpha_{m_{1}}^{(1)}\right)\right)\right)\right)\right)\right)\right) \vee \\
&\left(\alpha _ { m _ { 1 } } ^ { ( 1 ) } \wedge \left(\left(\alpha_{1}^{(2)} \wedge\left(\gamma_{1}^{(2)} \vee\left(\alpha_{2}^{(2)} \wedge \cdots \vee\left(\alpha_{m_{2}-1}^{(2)} \wedge\left(\gamma_{m_{2}-1}^{(2)} \vee \alpha_{m_{2}}^{(2)}\right)\right)\right)\right) \vee\right.\right.\right. \\
&\left.\left.\left(\alpha_{m_{1}+1}^{(1)} \wedge\left(\gamma_{m_{1}+1}^{(1)} \vee\left(\alpha_{m_{1}+2}^{(1)} \wedge \cdots \vee\left(\alpha_{m_{1}-1}^{(1)} \wedge \gamma_{m_{1}-1}^{(1)}\right)\right)\right)\right)\right)\right) .
\end{aligned}
$$

We likewise define the $L$-elements $\eta_{0}^{(i)}$ for all $1 \leq i \leq t$, with each containing all the $\alpha_{m_{i}}^{(i)}$ edges of rank 0 . Note that the long expression above is not to be read as one would initially think: it is not a join of four subexpression, but two, and each of those two is a meet of $\alpha_{m_{1}}^{(1)}$ and an other (lengthy) expression.

We recursively define $L$-elements $\eta_{n}^{(i)} \leq \alpha_{m_{i}}^{(i)}$ for all $1 \leq i \leq t$ and all nonnegative integer $n$ : for $n=0$ they are already defined, and for $n>0$ we set

$$
\begin{aligned}
& \eta_{n}^{(i)}:= \\
&\left(\alpha _ { m _ { i } } ^ { ( i ) } \wedge \left(\left(\alpha_{1}^{(i+1)} \wedge\left(\gamma_{1}^{(i+1)} \vee\left(\alpha_{2}^{(i+1)} \wedge \cdots \vee\left(\alpha_{m_{i+1}-1}^{(i+1)} \wedge\left(\gamma_{m_{i+1}-1}^{(i+1)} \vee \eta_{n-1}^{(i+1)}\right)\right)\right)\right)\right) \vee\right.\right.\left.\left(\alpha_{m_{i}+1}^{(i)} \wedge\left(\gamma_{m_{i}+1}^{(i)} \vee\left(\alpha_{m_{i}+2}^{(i)} \wedge \cdots \vee\left(\alpha_{m_{i}-1}^{(i)} \wedge\left(\gamma_{m_{i}-1}^{(i)} \vee \alpha_{m_{i}}^{(i)}\right)\right)\right)\right)\right)\right) \vee \\
&\left(\alpha _ { m _ { i } } ^ { ( i ) } \wedge \left(\left(\alpha_{1}^{(i+1)} \wedge\left(\gamma_{1}^{(i+1)} \vee\left(\alpha_{2}^{(i+1)} \wedge \cdots \vee\left(\alpha_{m_{i+1}-1}^{(i+1)} \wedge\left(\gamma_{m_{i+1}-1}^{(i+1)} \vee \alpha_{m_{i+1}}^{(i+1)}\right)\right)\right)\right)\right) \vee\right.\right. \\
&\left.\quad\left(\alpha_{m_{i}+1}^{(i)} \wedge\left(\gamma_{m_{i}+1}^{(i)} \vee\left(\alpha_{m_{i}+2}^{(i)} \wedge \cdots \vee\left(\alpha_{m_{i}-1}^{(i)} \wedge\left(\gamma_{m_{i}-1}^{(i)} \vee \eta_{n-1}^{(i)}\right)\right)\right)\right)\right)\right)
\end{aligned}
$$

If $\left(x_{1}, x_{2}\right)$ is an $\alpha_{m_{i}}^{(i)}$ edge $\left(x_{1}, x_{2}\right)$ of $\operatorname{rank} n$, then in its canonical realization all the $\alpha_{m_{i}}^{(i)}$ and $\alpha_{m_{i+1}}^{(i+1)}$ edges except perhaps one have rank at most $n-1$. From this fact it is easily provable by induction that $\left(x_{1}, x_{2}\right) \in \eta_{n}^{(i)}$.

As $\alpha_{m_{i}}^{(i)}$ is a completely join irreducible element of $\mathbf{L}$, and it is the union of all $\eta_{n}^{(i)}$ (as they form an increasing chain, and all $\alpha_{m_{i}}^{(i)}$ edge has finite rank), there is an $n_{i}$ so that $\alpha_{m_{i}}^{(i)}=\eta_{n_{i}}^{(i)}$. It can be supposed that among all the $n_{i}, n_{1}$ is (one of) the smallest.

As $\eta_{n_{1}}^{(1)}$ is defined as the join of two elements of $L$ that are smaller or equal than $\alpha_{m_{1}}^{(1)}$, and $\alpha_{m_{1}}^{(1)}$ is join irreducible in $\mathbf{L}, \alpha_{m_{1}}^{(1)}$ is equal to either

$$
\begin{aligned}
& \alpha_{m_{1}}^{(1)} \wedge\left(\left(\alpha_{1}^{(2)} \wedge\left(\gamma_{1}^{(2)} \vee\left(\alpha_{2}^{(2)} \wedge \cdots \vee\left(\alpha_{m_{2}-1}^{(2)} \wedge\left(\gamma_{m_{2}-1}^{(2)} \vee \eta_{n_{1}-1}^{(2)}\right)\right)\right)\right) \vee\right.\right. \\
&\left.\left(\alpha_{m_{1}+1}^{(1)} \wedge\left(\gamma_{m_{1}+1}^{(1)} \vee\left(\alpha_{m_{1}+2}^{(1)} \wedge \cdots \vee\left(\alpha_{m_{1}-1}^{(1)} \wedge\left(\gamma_{m_{1}-1}^{(1)} \vee \alpha_{m_{1}}^{(1)}\right)\right)\right)\right)\right)\right)
\end{aligned}
$$

or

$$
\begin{aligned}
& \alpha_{m_{1}}^{(1)} \wedge((\left.\alpha_{1}^{(2)} \wedge\left(\gamma_{1}^{(2)} \vee\left(\alpha_{2}^{(2)} \wedge \cdots \vee\left(\alpha_{m_{2}-1}^{(2)} \wedge\left(\gamma_{m_{2}-1}^{(2)} \vee \alpha_{m_{2}}^{(2)}\right)\right)\right)\right)\right) \vee \\
&\left.\left(\alpha_{m_{1}+1}^{(1)} \wedge\left(\gamma_{m_{1}+1}^{(1)} \vee\left(\alpha_{m_{1}+2}^{(1)} \wedge \cdots \vee\left(\alpha_{m_{1}-1}^{(1)} \wedge\left(\gamma_{m_{1}-1}^{(1)} \vee \eta_{n_{1}-1}^{(1)}\right)\right)\right)\right)\right)\right) .
\end{aligned}
$$


From both equalities follows an inequality of the type

$$
\alpha_{m_{1}}^{(1)} \leq\left(\alpha_{1}^{(2)} \wedge \delta_{1}\right) \vee\left(\alpha_{m_{1}+1}^{(1)} \wedge \delta_{2}\right)
$$

Recall the definition of the $\alpha_{j}^{(i)}$ to see that a consequence of this is that $\alpha_{1}^{(2)} \leq \delta_{1}$ and $\alpha_{m_{1}+1}^{(1)} \leq \delta_{2}$.

It follows from the $\alpha_{1}^{(2)} \leq \delta_{1}$ type inequality in the first case that

$$
\alpha_{m_{1}-1}^{(1)} \leq \gamma_{m_{1}-1}^{(1)} \vee \eta_{n_{1}-1}^{(1)}
$$

and in the second that

$$
\alpha_{m_{1}-1}^{(1)} \leq \gamma_{m_{1}-1}^{(1)} \vee \eta_{n_{1}-1}^{(1)} .
$$

Both are impossible: by the choice of $n_{1}, \eta_{n_{1}-1}^{(2)} \leq\left(\alpha_{m_{2}}^{(2)}\right)^{*}$ and $\eta_{n_{1}-1}^{(1)}<\left(\alpha_{m_{1}}^{(1)}\right)^{*}$, so either of these inequalities contradicts the fact that $\alpha_{j}^{(i)} \not \leq \gamma_{j}^{(i)} \vee\left(\alpha_{j+1}^{(i)}\right)^{*}$ is satisfied by all possible $i$ and $j$.

Problem 3.8. Is it true that $\mathbf{L}$ is representable if $E_{\mathbf{K}}$ contains no cycle for any $\mathbf{K} \leq \mathbf{L}$ ? Is it true if, moreover, $\mathbf{L}$ is finite?

Here is an overview of the known properties of the class of representable lattices.

Theorem 3.9. For the class $\mathcal{R}$ of lattices representable with DCC posets:

- $\mathcal{R}$ is closed to taking sublattices and products,

- $\mathcal{R}$ does not contain the lattice $M_{3}$,

- $\mathcal{R}$ contains all lower bounded lattices,

- $\mathcal{R}$ is not contained in $S D_{\vee}$,

- it is algorithmically decidable if a finite lattice $\mathbf{L}$ is in $\mathcal{R}$,

- if a lattice $\mathbf{L}$ is in $\mathcal{R}$, then $E_{\mathbf{L}}$ contains no circle.

Proof. The last two statements have been proved. $M_{3}$ fails the condition of the last statement, because the three middle elements form a D-cycle, and there is an E-loop on that cycle. As it was mentioned in the introduction, all lower bounded lattices are representable with posets satisfying both DCC and ACC by Theorem 4.6. of [29].

Take the following three posets on the set $\mathbb{N} \cup\{\infty\}$ :

- $\alpha:=\{(a, \infty): a$ is even. $\} \cup\{(c, c): c \in \mathbb{N} \cup\{\infty\}\}$

- $\beta:=\{(b, \infty): a$ is odd. $\} \cup\{(c, c): c \in \mathbb{N} \cup\{\infty\}\}$

- $\gamma:=\left\{(x, y) \in \mathbb{N}^{2}: x \leq y\right\} \cup\{(\infty, \infty)\}$ 
These posets generate a (six-element) lattice of DCC-posets (their join is the natural linear order on $\mathbb{N} \cup\{\infty\})$. That lattice is not join semidistributive as $\alpha \vee \gamma=\beta \vee \gamma>\gamma=(\alpha \wedge \beta) \vee \gamma$.

The only item left is that $\mathcal{R}$ is closed to direct products. If $\mathbf{L}_{i} \in \mathcal{R}$ for all $i \in I$ such that $\mathbf{L}_{i}$ is represented by DCC-posets on $X_{i}$ (which can be assumed to be disjoint), $\prod_{i \in I} \mathbf{L}_{i}$ can be represented on $\bigcup_{i \in I} X_{i}$ : the element $\left(l_{i}\right)_{i \in I}$ will be represented by the (disjoint) union of the posets representing the individual $l_{i}$.

Problem 3.10. Is it true that $\mathcal{R}$ contains $S D_{\vee}$ ? Is there a nontrivial lattice quasi-identity satisfied by all members of $\mathcal{R}$ ? 


\section{Chapter 4}

\section{Overview of tame congruence theory}

In this chapter, we go over those elements of tame congruence theory we will generalize for quasiorders, as well as those that will be otherwise needed later. We base everything here on [21], even when not noted.

\subsection{Minimal algebras and tame quotients}

Definition 4.1. A pair of elements $\left(l_{1}, l_{2}\right)$ of a lattice is called a quotient of that lattice if $l_{1}<l_{2}$, and a prime quotient if $l_{1} \prec l_{2}$. If $l_{1} \leq l_{3}<l_{4} \leq l_{2}$, then $\left(l_{3}, l_{4}\right)$ is a subquotient of $\left(l_{1}, l_{2}\right)$.

The quotients $\left(l_{1}, l_{2}\right)$ and $\left(l_{3}, l_{4}\right)$ are perspective if either $l_{2} \wedge l_{3}=l_{1}$ and $l_{2} \vee l_{3}=l_{4}$ or $l_{1} \wedge l_{4}=l_{3}$ and $l_{1} \vee l_{4}=l_{2}$ (equivalently, $l_{1}, l_{2}, l_{3}, l_{4}$ form a sublattice isomorphic to the direct square of the two-element chain). The two quotients are projective if there is a sequence of quotients beginning with the first and ending with the second such that each successive pair is perspective.

Two quotients are prime perspective if both are prime quotients and they are perspective. They are prime projective if there is a sequence of prime quotients beginning with the first and ending with the second such that each successive pair is perspective. (Note that prime projectivity is not projectivity restricted to prime quotients.)

In this chapter, $\mathbf{A}$ is always a finite algebra, and $\alpha<\beta$ are quasiorders of it.

Definition 4.2. For any set $U \subseteq A$, the algebra $\left.\mathbf{A}\right|_{U}$ is an algebra with underlying set $U$, whose set of basic operations is the set of all polynomials of $\mathbf{A}$ to which $U$ is closed.

For a binary relation $\delta$ on $A,\left.\delta\right|_{U}$ denotes the binary relation $\delta \cap U^{2}$ on $U$. Sometimes, if it does not cause confusion, we write $\delta$ instead of $\left.\delta\right|_{U}$. 
Proposition 4.3. For any $U \subseteq A,\left.\mathbf{A}\right|_{U}$ is an algebra in which any term and any polynomial is a basic operation.

If $\delta$ is compatible and reflexive on $\mathbf{A}$, then $\left.\delta\right|_{U}$ is compatible on $\left.\mathbf{A}\right|_{U}$. Thus $\left.\delta \mapsto \delta\right|_{U}$ induces a mapping from Quo $\mathbf{A}$ to Quo $\left.\mathbf{A}\right|_{U}$ (and from Con $\mathbf{A}$ to $\left.\operatorname{Con} \mathbf{A}\right|_{U}$ ), these mappings are meet homomorphisms.

If there is an idempotent unary polynomial e such that $e(A)=U$, then $\left.\delta \mapsto \delta\right|_{U}$ is a surjective lattice homomorphism from Quo $\mathbf{A}$ to Quo $\left.\mathbf{A}\right|_{U}$ (and thus also from $\operatorname{Con} \mathbf{A}$ to $\left.\left.\operatorname{Con} \mathbf{A}\right|_{U}\right)$.

Definition 4.4. A set $U \subseteq A$ is $(\alpha, \beta)$-minimal if there is a unary polynomial $p$ such that $p(A)=U$ and $p(\beta) \nsubseteq \alpha$ (that is, there exists $(x, y) \in \beta$ with $(p(x), p(y)) \notin \alpha)$, but there is no $q \in \operatorname{Pol}_{1} \mathbf{A}$ such that $q(A) \subsetneq U$ and $q(\beta) \nsubseteq \alpha$.

The set of all $(\alpha, \beta)$-minimal sets of $\mathbf{A}$ is denoted by $M(\alpha, \beta)$.

$\mathbf{A}$ is an $(\alpha, \beta)$-minimal algebra if $A$ is an $(\alpha, \beta)$-minimal set.

Finally, A is considered $(\gamma, \gamma)$-minimal for all $\gamma \in$ Quo A.

The last part of the definition was only mentioned because technically, $(\gamma, \gamma)$ is not a quotient of Quo A. It is completely in line with the rest of the definition otherwise.

Proposition 4.5. If $U$ is an $(\alpha, \beta)$-minimal set, then $\left.\mathbf{A}\right|_{U}$ is an $(\alpha, \beta)$-minimal algebra.

The next lemma is immediate from the definition of minimality.

Lemma 4.6. Suppose $\mathbf{A}$ is finite, $\alpha, \beta \in$ Quo $\mathbf{A}$ such that $\alpha<\beta$ and $\mathbf{A}$ is $(\alpha, \beta)$-minimal. Then $\mathbf{A}$ is also

- $\left(\alpha^{-1}, \beta^{-1}\right)$-minimal.

- $(\alpha \wedge \gamma, \beta \wedge \delta)$-minimal and $(\alpha \vee \gamma, \beta \vee \delta)$-minimal for any quasiorder quotient $(\gamma, \delta)$ such that $\mathbf{A}$ is also $(\gamma, \delta)$-minimal.

- $(\gamma, \delta)$-minimal whenever $(\gamma, \delta)$ is a subquotient of $(\alpha, \beta)$.

Corollary 4.7. Suppose that $(\alpha, \beta)$ and $(\gamma, \delta)$ are projective quotients of Quo $\mathbf{A}$. Then the $(\alpha, \beta)$-minimal and the $(\gamma, \delta)$-minimal sets of $\mathbf{A}$ coincide.

Lemma 4.8. Suppose that an algebra $\mathbf{A}$ is minimal with respect to one of its quasiorder quotients. Then $\mathbf{A}$ is also minimal with respect to either a congruence quotient or a quotient whose quasiorders have coinciding congruence parts.

Proof. Choose $\beta \in$ Quo $\mathbf{A}$ so that there is a quasiorder $\alpha$ such that $\mathbf{A}$ is $(\alpha, \beta)$ minimal, and $\beta$ is minimal among such quasiorders. According to Lemma 4.6, for any $\gamma \in$ Quo A either $\gamma \geq \beta$ or $\alpha \wedge \gamma=\beta \wedge \gamma$.

If $\beta$ is a congruence, take $\gamma=\alpha^{-1}<\beta$ to deduce $\alpha \wedge \alpha^{-1}=\beta \wedge \alpha^{-1}=$ $(\beta \wedge \alpha)^{-1}=\alpha^{-1}$, whence $\alpha$ is a congruence. If $\beta$ is not a congruence, choosing $\gamma=\beta^{-1}$ yields that the congruence part of $\beta$ is in $\alpha$, therefore $\alpha^{*}=\beta^{*}$. 
Definition 4.9. The pair $(\alpha, \beta)$ is called a quasiorder tame quotient (congruence tame quotient) if there is an $(\alpha, \beta)$-minimal set $U$ and an idempotent unary polynomial $e$ such that $e(A)=U$, and $\left.\alpha\right|_{U}<\left.\delta\right|_{U}<\left.\beta\right|_{U}$ for all $\alpha<\delta<\beta$ in Quo A (in Con A).

We will use only "tame quotient" henceforth because of the following fact.

Proposition 4.10. If $\alpha$ and $\beta$ are congruences, then $(\alpha, \beta)$ is a quasiorder tame quotient iff it is a congruence tame quotient.

Proof. Suppose that $(\alpha, \beta)$ is congruence tame, with $U$ and $e$ as in Definition 4.9. If there is a quasiorder $\delta$ such that $\alpha<\delta<\beta$ and $\left.\delta\right|_{U}=\left.\alpha\right|_{U}$ (resp. $\left.\left.\delta\right|_{U}=\left.\beta\right|_{U}\right)$, then $\nu:=\delta \vee \delta^{-1}$ (resp. $\left.\nu:=\delta^{*}\right)$ is a congruence with $\alpha<\nu \leq \beta$ (resp. $\alpha \leq \nu<\beta$ ) and $\left.\nu\right|_{U}=\left.\alpha\right|_{U}$ (resp. $\left.\nu\right|_{U}=\left.\beta\right|_{U}$, because of Proposition 4.3). By the $(\alpha, \beta)$-minimality of $U, \nu$ cannot equal $\beta$ (resp. cannot equal $\alpha$ ). This contradicts the assumption for $U$. Therefore $(\alpha, \beta)$ is also quasiorder tame. The other direction is obvious.

The following is parts of Theorems 2.8. and 2.11. of [21] stated to quasiorders. The proofs there can be applied word-for-word, as they do not use symmetry.

Theorem 4.11. If $\alpha \prec \beta$, then $(\alpha, \beta)$ is tame.

If $(\alpha, \beta)$ is tame, and $U$ and $V$ are $(\alpha, \beta)$-minimal sets, then

- there is an idempotent unary polynomial e such that $e(A)=U$ and $e(\beta) \nsubseteq$ $\alpha$,

- there are unary polynomials $p, q \in \operatorname{Pol}_{1} \mathbf{A}$ such that $p(U)=V, q(V)=U$, $q \circ p$ is identical on $U$, and $p \circ q$ is identical on $V$,

- the algebras $\left.\mathbf{A}\right|_{U}$ and $\left.\mathbf{A}\right|_{V}$ are isomorphic by $p$ (with the assumption that their signatures are fixed so that the basic operation $s\left(x_{1}, \ldots, x_{k}\right)$ of $\left.\mathbf{A}\right|_{U}$ corresponds to the basic operation $p\left(s\left(q\left(x_{1}\right), \ldots, q\left(x_{k}\right)\right)\right)$ of $\left.\left.\mathbf{A}\right|_{V}\right)$.

This theorem enables one to choose a single $(\alpha, \beta)$-minimal set without losing generality in most cases.

\subsection{Types in the congruence lattice}

In this section, $\alpha$ and $\beta$ are congruences of the finite algebra $\mathbf{A}$, and $\alpha \prec \beta$ in the congruence lattice (and so $(\alpha, \beta)$ is a tame quotient by Theorem 4.11).

Definition 4.12. A final algebra $\mathbf{B}$ is minimal, if it is $\left(0_{\mathbf{B}}, 1_{\mathbf{B}}\right)$-minimal.

Theorem 4.13. ([27]) The polynomial clone of a minimal algebra coincides with one of the following: 
1. a clone generated by unary operations,

2. the polynomial clone of a vector space (with the same underlying set),

3. the set of all operations on it underlying set,

4. the polynomial clone of a two-element lattice (with the same underlying set),

5. the polynomial clone of a two-element semilattice (with the same underlying set).

Definition 4.14. A minimal algebra is of type 1 (unary type), type 2 (affine type), type 3 (Boolean type), type 4 (lattice type), or type 5 (semilattice type), depending on which of the above five categories its polynomial clone falls into.

Definition 4.15. A $\beta$-class that is not an $\alpha$-class is called an $(\alpha, \beta)$-trace of $\mathbf{A}$. The union of the $(\alpha, \beta)$-traces are called the $(\alpha, \beta)$-body, the complement of the $(\alpha, \beta)$-body the $(\alpha, \beta)$-tail of $\mathbf{A}$.

A has no tail if for every $\mu \prec \nu$ in Con $\mathbf{A}$ the $(\mu, \nu)$-tail of every $(\mu, \nu)$ minimal set $U$ is empty. A variety has no tail if every finite algebra in it has no tail.

Theorem 4.16. ([21], Lemmas 4.13. and 4.20.) For any $\alpha, \beta$-minimal set $U$ of $\mathbf{A}$ and $\left(\left.\alpha\right|_{U},\left.\beta\right|_{U}\right)$-trace $N$ of $\left.\mathbf{A}\right|_{U}$, the algebra $\left.\mathbf{A}\right|_{N} /\left.\alpha\right|_{N}$ is a minimal algebra, and its type does not depend on the choice of $U$ and $N$.

With this theorem, a prime quotient in the congruence lattice is given a type:

Definition 4.17. The type of $(\alpha, \beta)$, denoted by typ $(\alpha, \beta)$, is the type of $\left.\mathbf{A}\right|_{N} /$ $\left.\alpha\right|_{N}$ for any $\left(\left.\alpha\right|_{U},\left.\beta\right|_{U}\right)$-trace $N$ of $\left.\mathbf{A}\right|_{U}$ for any $\alpha, \beta$-minimal set $U$ of $\mathbf{A}$.

For any $i \in\{1,2,3,4,5\}$, an algebra omits type $i$ if none of its prime congruence quotients has type $i$. A variety omits type $i$ if all of its finite members do.

This so-called "labeling" of the congruence lattice has the following properties:

Theorem 4.18. - if $\theta \in \operatorname{Con} \mathbf{A}$, then the types of covering pairs in $\operatorname{Con}(\mathbf{A} /$ $\theta$ ) are the same as the types of the corresponding pairs in Con $\mathbf{A}$ (pairs over the congruence $\theta$ ),

- any type depends only on the polynomial clone of the algebra, and not on the principal operations or the terms,

- prime projective pairs have the same type,

- the solvability relation on $\operatorname{Con} \mathbf{A}$ ( $\mu$ and $\nu$ are in the same class if the interval $[\mu \wedge \nu, \mu \vee \nu]$ omits types 3, 4, and 5) is a lattice congruence, and factoring out with it gives an upper bounded lattice. 
The following definition is, for technical reasons, more liberal than the corresponding Definition 4.16 of [21]. Also, this is only part in this section that is about quasiorders rather than congruences.

Definition 4.19. A pseudo-meet operation for an element $a$ of an algebra is any binary polynomial $p$ which satisfies the equations $p(a, x)=p(x, a)=p(x, x)=x$.

For quasiorders $\gamma<\delta$, a pseudo-meet operation for the quotient $(\gamma, \delta)$ is a pseudo-meet operation for a source or a target of a $\delta \backslash \gamma$-edge. A pseudo-meetpseudo-join pair for this quotient is a collection of two pseudo-meet operations, one for the source, one for the target of a $\delta \backslash \gamma$-edge.

Proposition 4.20. ([21], Lemmas 4.15. and 4.17.) If $(\gamma, \delta)$ is a congruence prime quotient of type 3,4 or 5 , and $\mathbf{A}$ is $(\gamma, \delta)$-minimal, then there is a pseudomeet operation for this quotient. If $(\gamma, \delta)$ is of type 3 or 4 , then there is a pseudo-meet-pseudo-join pair for it. Furthermore, in this latter case there is a unique $(\gamma, \delta)$-trace of $\mathbf{A}$, which has exactly two-elements.

The remaining part of this section reviews the facts concerning the case when an algebra is minimal to a non-prime quotient. This is equivalent to it being minimal to all of the prime subquotients of this quotient.

Proposition 4.21. ([21], Theorem 4.23.) Suppose $\mathbf{A}$ is $(\mu, \nu)$-minimal, and $\mu \nprec \nu$ in $\mathbf{C o n} \mathbf{A}$. Then the interval $[\mu, \nu]$ omits types 3, 4, and 5.

Proposition 4.22. ([21], Lemma 4.20.) If $(\mu, \nu)$ is a congruence prime quotient of type 2, then there is an idempotent ternary polynomial $m$ such that for any $x$ that is in the $(\mu, \nu)$-body of $\mathbf{A}$, and any $y \in A, m(x, x, y)=m(y, x, x)=y$. (Consequently, $m$ is a Mal'tsev-operation on the $(\mu, \nu)$-body of the $\mathbf{A}$.)

Definition 4.23. A ternary term $m$ satisfying the conditions of Proposition 4.22 is called a pseudo-Mal'tsev term for $(\mu, \nu)$.

Lemma 4.24. Suppose that $\mu_{0} \prec \mu_{1} \prec \cdots \prec \mu_{n}$ in $\operatorname{Con} \mathbf{A}$, A is $\left(\mu_{0}, \mu_{n}\right)$ minimal, and $\operatorname{typ}\left(\mu_{i}, \mu_{i+1}\right)=2$ for all $i$. Then for each pair $(a, b) \in \mu_{n}$, there is a bijective unary polynomial of $\mathbf{A}$ mapping $a / \mu_{0}$ to $b / \mu_{0}$.

Proof. Suppose first that $n=1$. If $(a, b) \in \mu_{0}$, then the statement is obvious. Otherwise, $a$ and $b$ are in the $\left(\mu_{0}, \mu_{1}\right)$-body of the algebra, on which the pseudoMal'tsev operation $m$ acts as a Mal'tsev operation. Thus, the unary polynomial $m(a, x, b)$ maps $a$ into $b$ and $b$ into $a$. This polynomial is bijective, because it maps a $\mu_{1}$-edge $((a, b))$ into a $\mu_{1} \backslash \mu_{0}$-edge $((b, a))$, and $\mathbf{A}$ is $\left(\mu_{0}, \mu_{1}\right)$-minimal.

The statement for greater $n$ is a consequence of the $n=1$ case: there is a bijective polynomial that maps $a$ into an $a_{n-1}$ which is in the $\mu_{n-1}$-class of $b$, an other which maps $a_{n-1}$ into an $a_{n-2}$ which is in the $\mu_{n-2}$-class of $b$, and so on. The succession of all these polynomials maps $a$ into $a_{0}$, which is in the $\mu_{0}$-class of $b$. 


\subsection{Description of some Mal'tsev classes}

If $\mathbf{A}$ is not minimal to a congruence quotient $(\mu, \nu)$, then it is a frequently used tactic to take a $(\mu, \nu)$-minimal set $U$, and work with $\left.\mathbf{A}\right|_{U}$ instead of $\mathbf{A}$. This is informally called "descending to a $(\mu, \nu)$-minimal algebra". This change alters the variety generated by the algebra (it even alters the signature of the algebra). However, for certain Mal'tsev classes, if $\mathbf{A}$ is in this Mal'tsev class (or, equivalently, A has terms satisfying the identities of the Mal'tsev class), then $\left.\mathbf{A}\right|_{U}$ is also in such a Mal'tsev class (equivalently, $\left.\mathbf{A}\right|_{U}$ also has terms satisfying those identities).

Definition 4.25. A Mal'tsev class is characterized by linear identities if there are identities $p_{1} \approx q_{1}, \ldots, p_{k} \approx q_{k}$ characterizing it in the sense of Definition 2.20 such that each $p_{i}$ and $q_{1}$ are of the form $t_{j}\left(e_{1}, \ldots, e_{\text {ar } t_{j}}\right)$, where $e_{1}, \ldots, e_{\text {ar } t_{j}}$ are all (not necessarily different) projections. (The index $j$ can be different for different elements of $\left\{p_{1}, \ldots, p_{k}, q_{1}, \ldots, q_{k}\right\}$.)

For example, the identity expressing the commutativity of a binary operation $(p(x, y) \approx p(y, x))$ is linear, but the one expressing its associativity $((p(x, p(y, z)) \approx p(p(x, y), z))$ is not. This definition also considers the identity expressing the idempotency of an operation $(p(x, \ldots, x) \approx x)$ as linear. Note that this is not always the case when encountering the term "linear identity" in the literature. (And as an aside, sometimes "linear identity" can mean something altogether different.)

Proposition 4.26. If $\mathfrak{K}$ is a Mal'tsev-class characterized by linear identities, $\mathbf{A}$ is a finite algebra that generates a variety in $\mathfrak{K}$, and $(\alpha, \beta)$ is a tame quotient of Quo $\mathbf{A}$, then for any $(\alpha, \beta)$-minimal set $U$ the algebra $\left.\mathbf{A}\right|_{U}$ generates a variety in $\mathfrak{K}$.

Proof. By Theorem 4.11, there is an idempotent unary polynomial $e$ such that $e(A)=U$. For any $t \in \operatorname{Term}_{k} \mathbf{A}$ there is a term $\left.t^{\prime} \in \operatorname{Term}_{k} \mathbf{A}\right|_{U}$ defined by $t^{\prime}\left(x_{1}, \ldots, x_{k}\right)=e\left(t\left(x_{1}, \ldots, x_{k}\right)\right)$. It is straightforward to check that if the terms $p_{1}, \ldots, p_{k} \in$ Term $\mathbf{A}$ satisfy some linear identities, then the terms $p_{1}^{\prime}, \ldots, p_{n}^{\prime} \in$ $\left.\operatorname{Term} \mathbf{A}\right|_{U}$ also satisfy these identities.

There are connections between the congruence lattices of a variety, the congruence types appearing in the variety, and certain Mal'tsev classes characterized by linear identities the variety may fall into. The following theorems show some of the most important of these.

Theorem 4.27. For a locally finite variety $\mathcal{V}$, the following are equivalent:

1. the algebras of $\mathcal{V}$ have modular congruence lattices,

2. no algebra of $\mathcal{V}$ has a congruence lattice having a sublattice isomorphic to $\mathbf{N}_{5}$,

3. ([21], Theorem 8.5) $\mathcal{V}$ omits 1 and 5, and has no tail, 
4. $[8] \mathcal{V}$ admits Day-terms, that is, there are $d_{0}, \ldots, d_{n} \in \operatorname{Term}_{4} \mathcal{V}$ satisfying

$$
\begin{aligned}
x & \approx p_{0}(x, y, z, u) \\
d_{i}(x, y, y, x) & \approx x \text { for all } i \\
d_{i}(x, y, y, z) & \approx d_{i+1}(x, y, y, z) \text { for even } i \\
d_{i}(x, x, y, y) & \approx d_{i+1}(x, x, y, y) \text { for odd } i \\
d_{n}(x, y, z, u) & \approx u
\end{aligned}
$$

5. [14] $\mathcal{V}$ admits Gumm-terms, that is, there are $p_{0}, \ldots, p_{n}, q \in \operatorname{Term}_{3} \mathcal{V}$ satisfying

$$
\begin{aligned}
x & \approx p_{0}(x, y, z) \\
p_{i}(x, y, x) & \approx x \text { for all } i \\
p_{i}(x, y, y) & \approx p_{i+1}(x, y, y) \text { for even } i \\
p_{i}(x, x, y) & \approx p_{i+1}(x, x, y) \text { for odd } i \\
p_{n}(x, y, y) & \approx q(x, y, y) \\
q(x, x, y) & \approx y
\end{aligned}
$$

Theorem 4.28. For a locally finite variety $\mathcal{V}$, the following are equivalent:

1. $\mathcal{V}$ omits 1 and 2 ,

2. ([21], Theorem 9.10) the algebras of $\mathcal{V}$ have meet semidistributive congruence lattices,

3. ([21], Theorem 9.10) no algebra of $\mathcal{V}$ has a congruence lattice having a sublattice isomorphic to $\mathbf{M}_{3}$,

4. ([24], Theorem 2.8) $\mathcal{V}$ admits terms $p \in \operatorname{Term}_{3} \mathcal{V}$ and $q \in \operatorname{Term}_{4} \mathcal{V}$ satisfying

$$
\begin{aligned}
x & \approx p(x, x, x) \\
x & \approx q(x, x, x, x) \\
p(x, x, y) & \approx p(x, y, x) \approx p(y, x, x) \\
q(x, x, x, y) \approx q(x, x, y, x) & \approx q(x, y, x, x) \approx q(y, x, x, x) \\
p(x, x, y) & \approx q(x, x, x, y)
\end{aligned}
$$

Theorem 4.29. ([21], Theorem 9.11) For a locally finite variety $\mathcal{V}$, the following are equivalent:

1. $\mathcal{V}$ omits 1, 2, and 5,

2. the finite algebras of $\mathcal{V}$ have join semidistributive congruence lattices, 
3. $\mathcal{V}$ admits ternary terms $d_{0}, \ldots, d_{n}$ satisfying the following identities:

$$
\begin{aligned}
x & \approx d_{0}(x, y, z) \\
d_{i}(x, y, y) & \approx d_{i+1}(x, y, y) \text { for even } i \\
d_{i}(x, y, x) & \approx d_{i+1}(x, y, x) \text { for even } i \\
d_{i}(x, x, y) & \approx d_{i+1}(x, x, y) \text { for odd } i \\
d_{n}(x, y, z) & \approx z
\end{aligned}
$$




\section{Chapter 5}

\section{Quasiorder types in finite algebras}

In this chapter, we adapt the types given to congruence prime quotients for the quasiorder prime quotients. A more compact form of the definition can be seen in the Summary.

\subsection{Definition of types for quasiorder quotients}

Suppose that $\mathbf{A}$ is a finite algebra, $\alpha \prec \beta$ in Quo A. All the induced $(\alpha, \beta)$ minimal algebras of $\mathbf{A}$ are isomorphic, so it is sufficient to define the type of $(\alpha, \beta)$ in the case where $\mathbf{A}$ is $(\alpha, \beta)$-minimal (otherwise, the algebra will inherit the type of the algebra induced by a minimal set). We will differentiate between two cases.

Definition 5.1. Suppose $\mathbf{A}$ is $(\alpha, \beta)$-minimal, and $\alpha^{*} \neq \beta^{*}$. If $\alpha^{*} \prec \beta^{*}$ in $\operatorname{Con} \mathbf{A}$, then set $\operatorname{typ}(\alpha, \beta)=\operatorname{typ}\left(\alpha^{*}, \beta^{*}\right)$. Otherwise, set $\operatorname{typ}(\alpha, \beta)=1$.

This may seem a little heavy-handed, as there are algebras minimal to one of their non-prime quotients omitting 1 (the most basic example is a multidimensional vector space), but as the next proposition shows, these non-prime quotients cannot be the respective congruence parts of a quasiorder prime quotient.

Proposition 5.2. If $\alpha \prec \beta$ in Quo $\mathbf{A}$, $\mathbf{A}$ is $(\alpha, \beta)$-minimal, and there is a congruence $\gamma$ such that $\alpha^{*}<\gamma<\beta^{*}$, then the interval $\left[\alpha^{*}, \beta^{*}\right]$ does not omit type 1 , more precisely, $\operatorname{typ}\left(\alpha^{*}, \mu\right)=1$ for any congruence $\mu$ such that $\alpha^{*} \prec \mu<\beta^{*}$ holds in $\mathbf{C o n} \mathbf{A}$.

Proof. It follows from $\mu<\beta$ and $\mu \not \leq \alpha$ that $\alpha \vee \mu=\beta$. This means that there exists an $\alpha$-crossedge, i.e. an $\alpha$-edge that is between different $\mu$-classes, but lies in a single $\beta^{*}$-class. $\mathbf{A}$ is of course also $\left(\alpha^{*}, \beta^{*}\right)$-minimal, thus by Proposition 
4.21, the congruence interval $\left[\alpha^{*}, \beta^{*}\right]$ omits 3,4 , and 5 . Now assume that $\operatorname{typ}\left(\alpha^{*}, \mu\right)=2$. By Lemma 4.24, the source of the $\alpha$-crossedge can be mapped by a bijective unary polynomial into any $\alpha^{*}$-class of its $\beta^{*}$-class. Hence, there is an $\alpha$-crossedge from every $\alpha^{*}$-class in that $\beta^{*}$-class, and so there is an $\alpha$ crossedge from every element of that $\beta^{*}$-class. But that means that there is a circle of $\alpha$-crossedges, therefore, there is an $\alpha^{*}$-class intersecting $\mu$-classes, a contradiction.

Corollary 5.3. If a congruence quotient is a prime quotient in the quasiorder lattice, then its quasiorder and congruence types coincide.

Now assume $\alpha^{*}=\beta^{*}$. First it needs to be understood what $(\alpha, \beta)$-minimality means in this case.

Proposition 5.4. If $\alpha^{*}=\beta^{*}$ for the prime quasiorder quotient $(\alpha, \beta)$, and $(a, b)$ is a $\beta \backslash \alpha$-edge, then $a / \beta^{*} \prec \beta / \beta^{*} b / \beta^{*}$. Furthermore, if $(c, d)$ is an other $\beta \backslash \alpha$-edge, then $\left(a / \beta^{*}, b / \beta^{*}\right)$ and $\left(c / \beta^{*}, d / \beta^{*}\right)$ can be mapped into each other by an automorphism of the poset $\beta / \beta^{*}$.

Proof. Obviously, $a / \beta^{*} \neq b / \beta^{*}$. There are elements $u^{\prime}$ and $v^{\prime}$ in $A / \beta^{*}$ such that $a / \beta^{*} \leq u^{\prime} \prec v^{\prime} \leq b / \beta^{*}$ in $\beta / \beta^{*}$, and $\left(u^{\prime}, v^{\prime}\right) \notin \alpha / \beta^{*}$. Take any representants $u$ and $v$ of $u^{\prime}$ and $v^{\prime}$ respectively. As $\beta$ is the quasiorder generated by $\alpha \cup\{(a, b)\}$, there are elements $u=c_{0}, \ldots, c_{l}=v$ in $A$ such that each $\left(c_{i}, c_{i+1}\right)$ is either in $\alpha$, or is a polynomial image of the edge $(a, b)$. These edges cannot all be in $\alpha$, and by $(\alpha, \beta)$-minimality the one that is not in $\alpha$ is the image of $(a, b)$ by a bijective polynomial. The inverse of this polynomial maps $\left(u^{\prime}, v^{\prime}\right)$ into $(a /$ $\beta^{*}, b / \beta^{*}$ ), which finishes the proof (bijective polynomials are automorphisms of compatible relations).

The second statement is a result of the same argument: simply consider that $\beta$ is also generated by $\alpha \cup\{(c, d)\}$.

Definition 5.5. The enlargement of $\mathbf{A}$ by the quasiorder $\beta$ (denoted by $\mathbf{A}_{+}$) is the subalgebra of $\mathbf{A}^{3}$ consisting of triples $(a, b, c)$ satisfying $(a, b),(b, c) \in \beta$. For an arbitrary $\delta \in$ Quo $\mathbf{A}$, the enlargement of $\delta$ is a congruence of $\mathbf{A}_{+}$defined by

$$
\delta_{+}=\operatorname{Tr}\left(\left\{\left((a, b, c),\left(a, b^{\prime}, c\right)\right) \in \mathbf{A}_{+}^{2}:\left(b, b^{\prime}\right) \in \delta \cup \delta^{-1}\right\}\right) .
$$

Informally, two triples are in $\delta_{+}$if their first and last components coincide, and there is a $\delta \cup \delta^{-1}$-path in $A$ between their middle components that lies entirely between the shared first and last component in $\beta$. It is easy to see that $\delta_{+}$is indeed a congruence of $\mathbf{A}_{+}$.

Proposition 5.6. The mapping $\delta \mapsto \delta_{+}$

- is a $\vee$-homomorphism from Quo $\mathbf{A}$ into Con $\mathbf{A}_{+}$,

- maps a quasiorder $\delta \geq \beta$ into the product congruence $0_{\mathbf{A}} \times 1_{\mathbf{A}} \times 0_{\mathbf{A}}$ (so $\left(a_{1}, a_{2}, a_{3}\right) \stackrel{\delta_{+}}{\longleftrightarrow}\left(b_{1}, b_{2}, b_{3}\right)$ iff $a_{1}=b_{1}$ and $\left.a_{3}=b_{3}\right)$, 
- maps $\alpha$ and $\beta$ into different congruences, moreover,

$$
\begin{aligned}
\beta_{+} \backslash \alpha_{+}=\left\{((c, a, d),(c, b, d)): c \stackrel{\beta^{*}}{\longrightarrow} a \stackrel{\beta \backslash \alpha}{\longrightarrow} b \stackrel{\beta^{*}}{\longleftrightarrow} d\right\} \cup \\
\left\{((c, a, d),(c, b, d)): c \stackrel{\beta^{*}}{\longrightarrow} b \stackrel{\beta \backslash \alpha}{\longrightarrow} a \stackrel{\beta^{*}}{\longrightarrow} d\right\}
\end{aligned}
$$

Proof. The first two points are easy deductions from the definition. We will only prove the formula of the third point.

Any element of the right hand side is immediately in $\beta_{+}$. That they are not in $\alpha_{+}$follows from the fact that (by Proposition 5.4) $c / \beta^{*} \prec_{\beta / \beta^{*}} d / \beta^{*}$, so the elements of $A$ between $c$ and $d$ in $\beta$ are the elements of the $\beta^{*}$-blocks of $c$ and $d$. Among these elements, there cannot be an $\alpha \cup \alpha^{-1}$-path between $a$ and $b$, because that would mean that $(a, b) \in \alpha$.

Conversely, take an element $((c, a, d),(c, b, d))$ of $\beta_{+} \backslash \alpha_{+}$. Note that both $((c, a, d),(c, d, d))$ and $((c, d, d),(c, b, d))$ are in $\beta_{+}$. One of two must not be in $\alpha_{+}$. If it is the first, then $(a, d) \in \beta \backslash \alpha$, if the second, then $(b, d) \in \beta \backslash \alpha$. Similarly, either $(c, a)$ or $(c, b)$ is in $\beta \backslash \alpha$.

By the second statement of Proposition 5.4, it is impossible that both $(c, a)$ and $(a, d)$ are in $\beta \backslash \alpha$, as $\left(c / \beta^{*}, a / \beta^{*}\right)$ obviously cannot be mapped into $(a /$ $\left.\beta^{*}, d / \beta^{*}\right)$ by an automorphism of $A / \beta^{*}$. Likewise, $(c, b),(b, d) \in \beta \backslash \alpha$ is also impossible.

Assume $(a, d),(c, b) \in \beta \backslash \alpha$. By Proposition 5.4, $c / \beta^{*}$ can be mapped into $a / \beta^{*}$ by a $\beta / \beta^{*}$-automorphism. But because $(c, a)$ is in $\beta, c$ and $a$ are in the same $\beta^{*}$-block. Consequently, $(a, b) \in \beta \backslash \alpha$. By the same argument, $d$ and $b$ are in the same $\beta^{*}$-block. A similar argument shows that if $(b, d),(c, a) \in \beta \backslash \alpha$, then $c \stackrel{\beta^{*}}{\longrightarrow} b \stackrel{\beta \backslash \alpha}{\longrightarrow} a \stackrel{\beta^{*}}{\longrightarrow} d$.

In the case $\beta=1_{\mathbf{A}}, \mathbf{A}_{+}=\mathbf{A}^{3}$, and for all $\delta \in$ Quo $\mathbf{A}, \delta_{+}=0_{\mathbf{A}} \times(\delta \vee$ $\left.\delta^{-1}\right) \times 0_{\mathbf{A}}$. This gives an easy example showing that $\delta \mapsto \delta_{+}$is not generally a $\wedge$-homomorphism: take $\delta$ as any non-symmetric quasiorder, $\gamma=\delta^{-1}$, then

$$
\delta_{+} \wedge \gamma_{+}=0_{\mathbf{A}} \times\left(\delta \vee \delta^{-1}\right) \times 0_{\mathbf{A}}>0_{\mathbf{A}} \times\left(\delta \wedge \delta^{-1}\right) \times 0_{\mathbf{A}}=(\delta \wedge \gamma)_{+} .
$$

Definition 5.7. Suppose $\mathbf{A}$ is $(\alpha, \beta)$-minimal, and $\alpha^{*}=\beta^{*}$. If the interval $\left[\alpha_{+}, \beta_{+}\right]$in Con $\mathbf{A}_{+}$does not omit 4 , then set $\operatorname{typ}(\alpha, \beta)=4$, otherwise, $\operatorname{typ}(\alpha, \beta)=5$ if the interval does not omit 5 , and $\operatorname{typ}(\alpha, \beta)=1$ if it does.

Admittedly, this definition is premature: we will soon prove that the interval $\left[\alpha_{+}, \beta_{+}\right]$necessarily omits 2 and 3 , and cannot contain both types 4 and 5 . It is convenient, though, to be able to speak of quasiorder types even before this is proved. In particular, we want to highlight the following consequence of the definition:

Proposition 5.8. If a prime quasiorder quotient is of type 2 or 3 , then it is a *-quotient. 


\subsection{Properties of different types}

By the definition of the previous section, the type of a prime quasiorder quotient is inherited from the type of its congruence part, or the congruence types between the enlargements of the quasiorders. We will refer to a prime quasiorder quotient as *-quotient or +-quotient depending on whether the former or the latter is the case.

First we prove that a prime quotient being a + -quotient is equivalent to the congruence parts of the two quasiorders coinciding (this is true by definition only if the algebra is minimal to this quotient).

Proposition 5.9. A prime quotient $(\alpha, \beta)$ is a +-quotient iff $\alpha^{*}=\beta^{*}$.

Proof. We have to show that if the congruence parts of $\alpha$ and $\beta$ differ on $\mathbf{A}$, then they also differ on an $(\alpha, \beta)$-minimal set $M$ of $\mathbf{A}$. This follows from the fact (Proposition 4.3) that the restriction to $M$ is a lattice homomorphism from Quo A to Quo A $\left.\right|_{M}$, thus $\left.\left.\alpha\right|_{M} \vee \beta^{*}\right|_{M}=\left.\left(\alpha \vee \beta^{*}\right)\right|_{M}=\left.\beta\right|_{M}$, while $\left.\left.\alpha\right|_{M} \vee \alpha^{*}\right|_{M}=$ $\left.\alpha\right|_{M}$, so $\left.\alpha^{*}\right|_{M}$ and $\left.\beta^{*}\right|_{M}$ must differ.

The following lemma informally states that when "descending" to a minimal set of an enlargement, not too much "information" is lost, as the minimal set is at least as large as the original (minimal) algebra.

Lemma 5.10. If $\mathbf{A}$ is ( $\alpha, \beta)$-minimal for the +-quotient $(\alpha, \beta)$, then for any $\left(\alpha_{+}, \beta_{+}\right)$-minimal set $M$ of $\mathbf{A}_{+}$and any $c \in A, c$ is a middle component of one of the elements of $M$.

Proof. Take any polynomial $p \in \mathrm{Pol}_{1} \mathbf{A}_{+}$that does not map $\beta_{+}$into $\alpha_{+}$. It is easy to see that it acts componentwise, that is, there are $p_{1}, p_{2}, p_{3} \in \mathrm{Pol}_{1} \mathbf{A}$ such that $p(a, b, c)=\left(p_{1}(a), p_{2}(b), p_{3}(c)\right)$ for any $(a, b, c) \in A_{+}$. By the $(\alpha, \beta)$ minimality of $\mathbf{A}$, it is enough to show that $p_{2}$ does not map $\beta$ into $\alpha$. This is a consequence of Proposition 5.6: the middle components of the $\beta_{+} \backslash \alpha_{+}$-edges are in $(\beta \backslash \alpha) \cup(\beta \backslash \alpha)^{-1}$, thus if $p_{2}$ maps $\beta$ into $\alpha$, then $p$ will map these edges into $\alpha_{+}$-edges.

Now we can state the following crucial property of +-quotients.

Lemma 5.11. Suppose $\mathbf{A}$ is $(\alpha, \beta)$-minimal, where $(\alpha, \beta)$ is a +-quotient. Then for any $\alpha_{+} \leq \mu \prec \nu \leq \beta_{+}$(in Con $\mathbf{A}_{+}$), the type of the congruence quotient $(\mu, \nu)$ cannot be 2 or 3 . If the type is 5 , there is a pseudo-meet operation, if 4 , a pseudo-meet-pseudo-join pair for the quasiorder quotient $(\alpha, \beta)$.

Proof. If the type was 2 or 3 , there would be a polynomial of $\mathbf{A}_{+}$mapping a $\beta_{+} \backslash \alpha_{+}$edge $(x, y)$ into a $\beta_{+} \backslash \alpha_{+}$edge $\left(y^{\prime}, x^{\prime}\right)$ so that $x \stackrel{\alpha_{+}}{\longleftrightarrow} x^{\prime}$ and $y \stackrel{\alpha_{+}}{\longleftrightarrow} y^{\prime}$ (take any $\nu \backslash \mu$-edge of a $(\mu, \nu)$-minimal set). By Proposition 5.6, the middle component of this polynomial would map a $\beta \backslash \alpha$ edge into a $(\beta \backslash \alpha)^{-1}$ edge (in the case of type 2 , note that a $\nu$-block cannot contain more than two $\mu$-blocks). This contradicts $\beta \cap(\beta \backslash \alpha)^{-1}=\emptyset$, which is a consequence of the fact that $(\alpha, \beta)$ is a +-quotient. 
If the type of $(\mu, \nu)$ is 4 or 5 , take a $(\mu, \nu)$-minimal set $M$. There is a pseudo-meet operation (a pseudo-meet-pseudo-join pair in the case of type 4) for $(\mu, \nu)$ in $\mathbf{M}$ by Proposition 4.20. By Lemma 5.10, its middle component is a pseudo-meet operation (a pseudo-meet-pseudo-join pair) for $(\alpha, \beta)$, because it follows from Proposition 5.6 that the middle component of a $\nu \backslash \mu$ edge is either a $\beta \backslash \alpha$ or a $(\beta \backslash \alpha)^{-1}$ edge.

As a pseudo-meet operation for $\left(\gamma^{*}, \delta^{*}\right)$ is also one for $(\gamma, \delta)$ :

Corollary 5.12. If an algebra is minimal with respect to a prime quasiorder quotient of type 3,4 (of type 5), then there is a pseudo-meet-pseudo-join pair (a pseudo-meet operation) for it.

Lemma 5.13. If $\mathbf{A}$ is minimal to a (not necessarily prime) quasiorder quotient $(\alpha, \beta)$, and there is a pseudo-meet operation $p$ for $a \in A$, then all $\beta \backslash \alpha$-edges have a as either source or target.

Proof. For any $x_{0} \in A \backslash\{a\}$, the unary polynomials $p\left(x, x_{0}\right)$ and $p\left(x_{0}, x\right)$ are not bijective (mapping both $x_{0}$ and $a$ into $x_{0}$ ), therefore they map $\beta$ into $\alpha$. This means that for any $\left(d_{1}, d_{2}\right) \in \beta \backslash \alpha$, either $d_{1}=a$ or $d_{2}=a$, because otherwise, $d_{1}=p\left(d_{1}, d_{1}\right) \stackrel{\alpha}{\rightarrow} p\left(d_{1}, d_{2}\right) \stackrel{\alpha}{\rightarrow} p\left(d_{2}, d_{2}\right)=d_{2}$.

Corollary 5.14. If $\mathbf{A}$ is minimal to the type 4 (type 5) +-quotient $(\alpha, \beta)$, then $\beta \backslash \alpha$ contains only a single edge (contains edges with either a common source or a common target).

Proof. Suppose that $\left(a_{0}, b_{0}\right) \in \beta \backslash \alpha$ such that there is a pseudo-meet operation $p$ for $a_{0}$. Take an arbitrary $(a, b) \in \beta \backslash \alpha$, by Lemma 5.13 either $a=a_{0}$ or $b=a_{0}$. The second case is impossible by Proposition 5.4, because no automorphism of $\beta / \beta^{*}$ can map $a$ into $b$, as $(a, b)$ is a $\beta \backslash \beta^{*}$ edge. So all the $\beta \backslash \alpha$-edges have a shared source. If there is a pseudo-meet operation for $b_{0}$ instead, then all the $\beta \backslash \alpha$-edges will have a shared target. Finally, if there is a pseudo-meet-pseudojoin pair, then the $\beta \backslash \alpha$-edges must coincide. 5.

So, as was promised, the interval $\left[\alpha_{+}, \beta_{+}\right]$cannot contain both types 4 and

Corollary 5.15. If $\mathbf{A}$ is minimal to the type $4_{+}+$-quotient $(\alpha, \beta)$, then $\alpha_{+} \prec \beta_{+}$ in Con $\mathbf{A}_{+}$.

Proof. By Corollary 5.14, there is only one $\beta \backslash \alpha$-edge, obviously, both its source and target must be a singleton $\beta^{*}$-class. By Proposition 5.6, $\beta_{+} \backslash \alpha_{+}$is a single double edge.

Lemma 5.11 has a converse.

Lemma 5.16. If $\mathbf{A}$ is minimal to the +-quotient $(\alpha, \beta)$, and there is a pseudomeet operation for this quotient, then $\operatorname{typ}(\alpha, \beta) \in\{4,5\}$. If there is a pseudomeet-pseudo-join pair for this quotient, then $\operatorname{typ}(\alpha, \beta)=4$. 
Proof. We only prove the first statement of the lemma, as the second is much the same.

Take a pair of congruences $\alpha_{+} \leq \mu \prec \nu \leq \beta_{+}$of $\mathbf{A}_{+}$, and let $M$ be a $(\mu, \nu)$ minimal set. By Proposition 5.6, there is an element of $\beta_{+} \backslash \alpha_{+} \cap M^{2}$ in the form $((c, a, d),(c, b, d))$, where $(a, b) \in \beta \backslash \alpha$, and $(a, c),(b, d) \in \alpha^{*}$. By Lemma 5.13 , the pseudo-meet operation $p$ is for either $a$ or $b$. It may be assumed that $p(a, x)=p(x, a)=p(x, x)=x$ for all $x$.

There is an idempotent unary polynomial $e \in \mathrm{Pol}_{1} \mathbf{A}_{+}$such that $e\left(A_{+}\right)=M$ (Theorem 4.11). Define the binary polynomial $p^{\prime}$ on $\left.\mathbf{A}_{+}\right|_{M}$ with

$$
p^{\prime}\left(\left(x_{1}, x_{2}, x_{3}\right),\left(y_{1}, y_{2}, y_{3}\right)\right):=e\left(\left(p\left(x_{1}, y_{1}\right), p\left(x_{2}, y_{2}\right), p\left(x_{3}, y_{3}\right)\right)\right) .
$$

As $p$ is idempotent (as an operation) and $e$ is idempotent (not as an operation, but as a transformation: $\left.e^{2}=e\right), p^{\prime}$ is also an idempotent operation. Furthermore, $p^{\prime}((c, a, d),(c, b, d))=p^{\prime}((c, b, d),(c, a, d))=(c, b, d)$, so $p^{\prime}$ is a proper binary polynomial on an $\left(\alpha_{+}, \beta_{+}\right)$-trace of $M$. This means that typ $(\alpha, \beta) \neq 1$. As the type of a +-quotient cannot be 2 or 3 (Lemma 5.11), the proof is done.

We summarize this section in the following theorem:

Theorem 5.17. If $\mathbf{A}$ is minimal to the prime quotient $(\alpha, \beta)$, then the type of $(\alpha, \beta)$ is

- 3, iff $\beta \backslash \alpha$ is a single double edge, and there is a pseudo-meet-pseudo-join pair for it (this case is only possible if $\alpha^{*} \neq \beta^{*}$ ),

- 4 , iff $\beta \backslash \alpha$ is a single (directed) edge, and there is a pseudo-meet-pseudojoin pair for it,

- 5, iff there is a pseudo-meet operation for it, but not a pseudo-meetpseudo-join pair (and in this case, the pseudo-meet operation is for either the shared target or the shared source of all the $\beta \backslash \alpha$-edges),

- 2, iff $\left(\alpha^{*}, \beta^{*}\right)$ is a prime congruence quotient of type 2,

- 1 in any other case.

Proof. The +-quotient case is covered by Lemma 5.11, Lemma 5.16, and Proposition 5.8. In the ${ }^{*}$-case, the statement for type 2 is the first statement of Proposition 5.2. Finally, the statements for types 3,4 , and 5 for ${ }^{*}$-quotients can be easily deduced by Lemma 5.13 .

\subsection{Types in a quasiorder lattice}

There are two basic conditions about the labeling of congruence lattices: the first is that prime perspective quotients must have the same type, the second that the solvability and strong solvability relations must be congruences. The first is also true for the labelings of quasiorder lattices. 


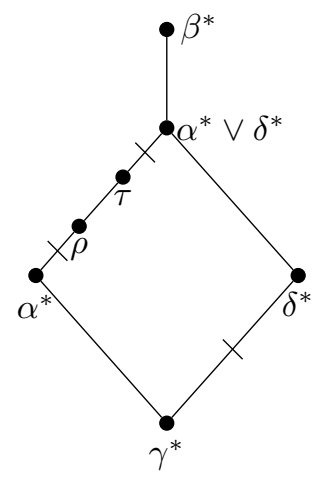

Figure 5.1: The subcase of Case 2 when $\operatorname{typ}(\alpha, \beta)=1$

Theorem 5.18. Suppose that $(\alpha, \beta)$ and $(\gamma, \delta)$ are prime projective quotients of Quo A. Then $\operatorname{typ}(\alpha, \beta)=\operatorname{typ}(\gamma, \delta)$.

Proof. Throughout the proof, we will use Theorem 5.17 without explicit references.

It can be assumed that the two quotients are prime perspective with $\beta<\delta$, this means that $\alpha \wedge \delta=\gamma$ and $\alpha \vee \delta=\beta$. As the minimal $(\alpha, \beta)$ and $(\gamma, \delta)$ sets of the algebra coincide (Corollary 4.7), it can also be assumed that $\mathbf{A}$ is both $(\alpha, \beta)$ - and $(\gamma, \delta)$-minimal. Note that if $(\alpha, \beta)$ is a + -quotient, then so is $(\gamma, \delta)$.

CASE 1: Both are +-quotients

In this case, by Proposition 5.8, both types are among 1, 4, and 5 .

If $\operatorname{typ}(\alpha, \beta)=4$, then $|\beta \backslash \alpha|=1$. As $\emptyset \neq \delta \backslash \gamma \subseteq \beta \backslash \alpha$, this means that $\delta \backslash \gamma=$ $\beta \backslash \alpha$, hence there is a pseudo-meet-pseudo-join pair for $(\gamma, \delta)$, so $\operatorname{typ}(\gamma, \delta)=4$.

If $\operatorname{typ}(\alpha, \beta)=5$, then, as there is a pseudo-meet operation for any $\beta \backslash \alpha$-edge, there is also one for $(\gamma, \delta) \operatorname{typ}(\gamma, \delta)=4$ is not possible, as it would mean that there is a pseudo-meet-pseudo-join pair for $(\gamma, \delta)$, and it would also be one to $(\alpha, \beta)$. Therefore $\operatorname{typ}(\gamma, \delta)=5$.

Finally, if $\operatorname{typ}(\alpha, \beta)=1$, there must not exist a pseudo-meet operation for $(\gamma, \delta)$, because it would also be one for $(\alpha, \beta)$, so $\operatorname{typ}(\gamma, \delta)=1$.

CASE 2: Both are ${ }^{*}$-quotients

First, assume that neither of the two types is 1 . Then $\alpha^{*} \prec \beta^{*}$, and $\gamma^{*} \prec \delta^{*}$ in the congruence lattice because of Proposition 5.2. Notice that $\alpha^{*} \wedge \delta^{*}=\gamma^{*}$ (as $\xi \mapsto \xi^{*}$ is a $\wedge$-homomorphism) and $\alpha^{*} \vee \delta^{*}=\beta^{*}$ (if $\alpha^{*} \vee \delta^{*}$ was $\alpha^{*}$, then $\alpha^{*} \wedge \delta^{*}$ would be $\left.\delta^{*}\right)$. Therefore, $\left(\alpha^{*}, \beta^{*}\right)$ and $\left(\gamma^{*}, \delta^{*}\right)$ are prime perspective congruence quotients, and by Theorem $4.18, \operatorname{typ}(\alpha, \beta)=\operatorname{typ}\left(\alpha^{*}, \beta^{*}\right)=\operatorname{typ}\left(\gamma^{*}, \delta^{*}\right)=$ $\operatorname{typ}(\gamma, \delta)$.

Now assume that $1=\operatorname{typ}(\gamma, \delta) \neq \operatorname{typ}(\alpha, \beta)$. Again, $\alpha^{*} \wedge \delta^{*}=\gamma^{*}$, and $\alpha^{*} \vee \delta^{*}=\beta^{*}$, because $\left(\alpha^{*}, \beta^{*}\right)$ is a prime congruence quotient. Choose a congruence $\eta$ such that $\gamma^{*} \prec \eta \leq \delta^{*}$. By Proposition 5.2, $\operatorname{typ}\left(\gamma^{*}, \eta\right)=1$. But by the congruence prime perspectivity of $\left(\alpha^{*}, \beta^{*}\right)$ and $\left(\gamma^{*}, \eta\right)$ and Theorem 4.18, $\operatorname{typ}(\alpha, \beta)=\operatorname{typ}\left(\alpha^{*}, \beta^{*}\right)=\operatorname{typ}\left(\gamma^{*}, \eta\right)$, a contradiction. 
Finally, assume that $\operatorname{typ}(\gamma, \delta) \neq \operatorname{typ}(\alpha, \beta)=1$. Choose a congruence $\tau$ such that $\alpha^{*} \leq \tau \prec \alpha^{*} \vee \delta^{*}$. The type of $\left(\tau, \alpha^{*} \vee \delta^{*}\right)$ cannot be 3 , 4, or 5 , because that (along with the $\left(\alpha^{*}, \beta^{*}\right)$-minimality of the algebra) would mean by Proposition 4.21 that $\tau=\alpha^{*}, \alpha^{*} \vee \gamma^{*}=\beta^{*}$, and $\operatorname{sotyp}\left(\alpha^{*}, \beta^{*}\right) \in\{3,4,5\}$. Hence, $\operatorname{typ}(\gamma, \delta)=\operatorname{typ}\left(\gamma^{*}, \delta^{*}\right)=\operatorname{typ}\left(\tau, \alpha^{*} \vee \delta^{*}\right)=2$, because $\left(\gamma^{*}, \delta^{*}\right)$ and $\left(\tau, \alpha^{*} \vee \delta^{*}\right)$ are prime perspective (It follows from Proposition 5.2 that $\left(\gamma^{*}, \delta^{*}\right)$ is a prime congruence quotient.)

Now choose a congruence $\rho$ such that $\alpha^{*} \prec \rho \leq \alpha^{*} \vee \delta^{*}$. (See Figure 5.1.) By Proposition 5.2, $\operatorname{typ}\left(\alpha^{*}, \rho\right)=1$. Consider an $\left(\alpha^{*}, \rho\right)$-trace $D$. Denote with $C$ the $\alpha^{*} \vee \delta^{*}$-class that contains $D$.

Notice that any $\alpha^{*}$-class of $C$ must contain an element of the $\left(\gamma^{*}, \delta^{*}\right)$-body of the algebra (otherwise, $C$ would be an $\alpha^{*}$-class, and that is not compatible with the fact that $C$ contains an $\left(\alpha^{*}, \rho\right)$-trace.). Consider the pseudo-Mal'tsev operation $d$ for the congruence quotient $\left(\gamma^{*}, \delta^{*}\right)$ that exists by Proposition 4.22. As any $\alpha^{*}$-class of $D$ contains an element that is in the $\left(\gamma^{*}, \delta^{*}\right)$-body, any $\alpha^{*}$ class of $D$, as well as $D$ itself, is a subalgebra with respect to the polynomial $d$ (because $d$ is idempotent on the $\left(\gamma^{*}, \delta^{*}\right)$-body). Also, $d$ acts as a Mal'tsevoperation on the $\alpha^{*}$-classes of $D$ : if $A_{1}$ and $A_{2}$ are two such classes, then there are $a_{1} \in A_{1}$ and $a_{2} \in A_{2}$ such that both $a_{1}$ and $a_{2}$ is in the $\left(\gamma^{*}, \delta^{*}\right)$-body, thus $d\left(a_{1}, a_{1}, a_{2}\right)=d\left(a_{2}, a_{1}, a_{1}\right)=a_{2}$, and any element of $d\left(A_{1}, A_{1}, A_{2}\right)$ or $d\left(A_{2}, A_{1}, A_{1}\right)$ must be in the same $\alpha^{*}$-class as $a_{2}$. This contradicts typ $\left(\alpha^{*}, \rho\right)=$ 1.

CASE 3: $(\alpha, \beta)$ is a *-quotient, while $(\gamma, \delta)$ is a + -quotient

By Proposition 5.8 , the type of $(\gamma, \delta)$ is 1,4 , or 5 .

If $\operatorname{typ}(\gamma, \delta)=4, \delta \backslash \gamma$ is a single edge $(a, b)$. As $\alpha^{*} \neq \beta^{*}$, there is an edge $(c, d) \in \beta^{*} \backslash \alpha$. There is an $\alpha-\delta$ path from $c$ to $d$, which is possible in only one fashion: if

$$
c \stackrel{\alpha}{\rightarrow} a \stackrel{\delta \backslash \gamma}{\longrightarrow} b \stackrel{\alpha}{\rightarrow} d .
$$

Consider the pseudo-meet operation $p$ for $a$ (so $p(a, x)=p(x, a)=p(x, x)=$ $x$ for all $x$ ), and the path

$$
c=p(c, a) \stackrel{\delta}{\rightarrow} p(c, b) \stackrel{\alpha}{\rightarrow} p(a, d)=d,
$$

to deduce that $c=a$. Likewise, deduce $d=b$ using the pseudo-meet operation for $b$. So the pseudo-meet-pseudo-join pair for $(\gamma, \delta)$ acts on the unique $\left(\alpha^{*}, \beta^{*}\right)$ trace as lattice operations, thus $\operatorname{typ}(\alpha, \beta)=4$ (the type is not 3 , because that would mean that $(d, c)$ is also in $\beta^{*} \backslash \alpha$, which would yield that $(d, c)$ is also coinciding with $(a, b))$.

If $\operatorname{typ}(\gamma, \delta)=5$, it can be assumed that the edges in $\delta \backslash \gamma$ share a common source $a$, and there is a pseudo-meet operation $p$ for it. As in the previous case, it can be deduced that for an arbitrary $(c, d) \in \beta^{*} \backslash \alpha, c$ is coinciding with $a$, furthermore, there is a $b \in A$ such that

$$
a \stackrel{\delta \backslash \gamma}{\longrightarrow} b \stackrel{\alpha}{\rightarrow} d .
$$

This means that there is a pseudo-meet operation for $(c, d)$, we need only to prove that it has no pseudo-join pair. 
Assume contrariwise that $q$ is a bijective polynomial such that $q(d, x)=$ $q(x, d)=q(x, x)=x$, hence $\operatorname{typ}(\alpha, \beta) \in\{3,4\}$, and thus $\left(\alpha^{*}, \beta^{*}\right)$ is a prime quotient of type 3 or 4 . Now consider

$$
a=q(a, a) \stackrel{\delta}{\rightarrow} q(a, b) \stackrel{\alpha}{\rightarrow} q(a, d)=a,
$$

to see that $q(a, b)$ is in the $\beta^{*}$-class of $a$, but that class only contains $a$ and $d$ by Proposition 4.20. If $q(a, b)=d$, then $(a, d) \in \delta \backslash \gamma$, which is a contradiction, as there is a pseudo-meet-pseudo-join pair for $(a, d)$. So $q(a, b)=a$, and likewise, $q(b, a)=a$, thus there is a pseudo-meet-pseudo-join pair for $(a, b)$, again a contradiction.

Finally, set $\operatorname{typ}(\gamma, \delta)=1$. If $\operatorname{typ}(\alpha, \beta) \in\{3,4,5\}$, then it can be assumed there is an edge $(a, b) \in \beta^{*} \backslash \alpha$ and a pseudo-meet operation $p$ for $a$. By Lemma 5.13 , taking an arbitrary $\delta \backslash \gamma$-edge, $a$ is either a source or a target of it, so there is a pseudo-meet operation for $(\gamma, \delta)$, which contradicts $\operatorname{typ}(\gamma, \delta)=1$.

If $\operatorname{typ}(\alpha, \beta)=2$, there is a pseudo-Mal'tsev operation $m$ for $\left(\alpha^{*}, \beta^{*}\right)$ by Proposition 4.22. Take an arbitrary edge $(a, b) \in \beta^{*} \backslash \alpha^{*}$, there is an $\alpha-\delta \backslash \gamma$ path from $a$ to $b$ :

$$
a \stackrel{\alpha}{\rightarrow} c_{1} \stackrel{\delta \backslash \gamma}{\longrightarrow} \ldots \stackrel{\alpha}{\rightarrow} c_{k} \stackrel{\delta \backslash \gamma}{\longrightarrow} b
$$

This path is entirely in $\beta$, and as it is between elements of the same $\beta^{*}$-class, it must lie entirely in that $\beta^{*}$-class. Therefore, $c_{1}$ and $c_{2}$ are in the $\left(\alpha^{*}, \beta^{*}\right)$ body, so $\left(c_{2}, c_{1}\right)=\left(m\left(c_{1}, c_{1}, c_{2}\right), m\left(c_{1}, c_{2}, c_{2}\right)\right) \in \delta$, and $\left(c_{1}, c_{2}\right) \in \delta^{*} \backslash \gamma^{*}$, which contradicts the fact that $(\gamma, \delta)$ is a + -quotient.

On the other hand, the concept of solvability does not extend to quasiorder lattices-at least, not in a natural way. Consider the semigroup $\mathbf{S}$ with the following multiplication table:

\begin{tabular}{l|llll} 
& 0 & 1 & 2 & 3 \\
\hline 0 & 0 & 0 & 0 & 0 \\
1 & 1 & 1 & 1 & 1 \\
2 & 0 & 1 & 2 & 2 \\
3 & 0 & 1 & 2 & 3
\end{tabular}

The usual definition of solvability ( $\alpha$ and $\beta$ being in the same block iff $\operatorname{typ}[\alpha \wedge \beta, \alpha \vee \beta] \subseteq\{1,2\})$ does not yield a congruence in this case. Neither does the definition of strong solvability ( $\alpha$ and $\beta$ being in the same block iff $\operatorname{typ}[\alpha \wedge \beta, \alpha \vee \beta] \subseteq\{1\})$.

Proposition 5.19. There are $\alpha, \beta, \gamma, \delta \in$ Quo S such that $\alpha \prec \beta, \gamma \prec \delta<\beta \vee \gamma$, $\operatorname{typ}(\alpha, \beta)=1$, and $\operatorname{typ}(\gamma, \delta)=5$.

Proof. Put

$$
\begin{aligned}
\alpha=0_{\mathbf{S}}, \bar{\beta}=\{(1,0)\}, \bar{\gamma}=\{(0,1),(0,2),(0,3),(2,1)\} & \\
\bar{\delta} & =\{(0,1),(0,2),(0,3),(2,1),(2,3)\},
\end{aligned}
$$




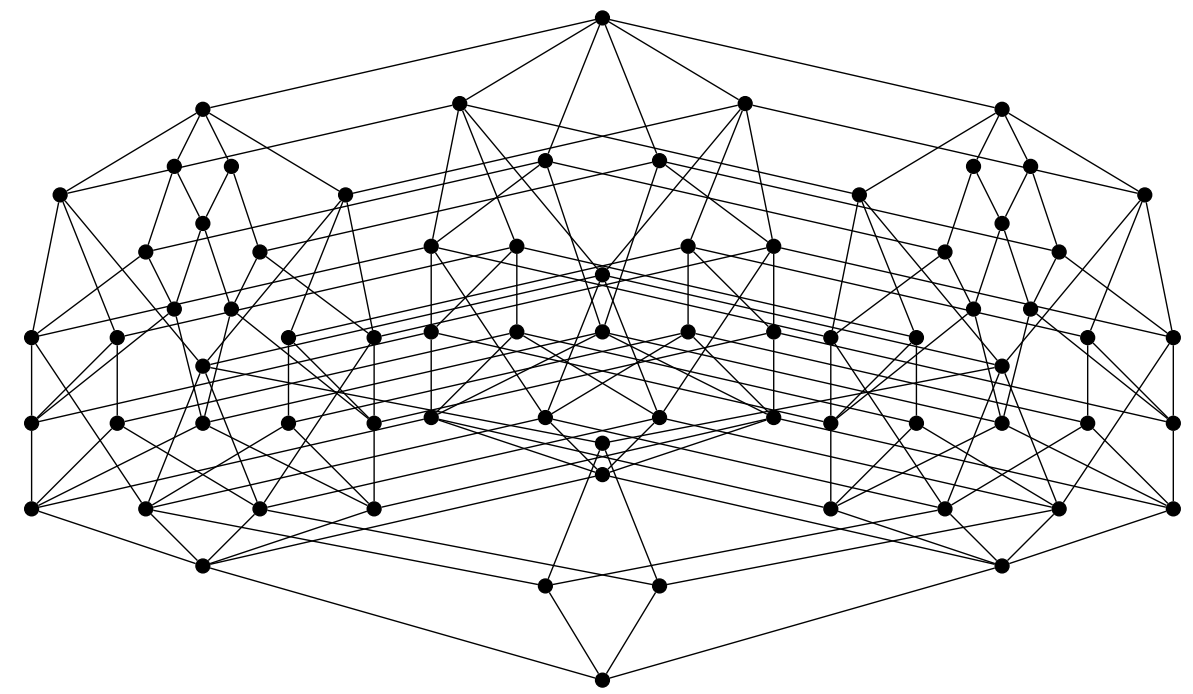

Figure 5.2: Quo $\mathbf{S}$

with $\bar{\omega}$ denoting the set of non-loop edges of a reflexive binary relation $\omega$.

As the set $\{0,1\}$ is $(\alpha, \beta)$-minimal, and the algebra induced on it is trivial, $\operatorname{typ}(\alpha, \beta)=1$. $\mathbf{S}$ is itself a $(\gamma, \delta)$-minimal algebra, $(2,3) \in \delta \backslash \gamma$, and the multiplication is a pseudo-meet operation for 3 (with there being no pseudo-meet operation for 2$)$, so $\operatorname{typ}(\gamma, \delta)=5$ by Theorem 5.17 .

One may try to alter the definition of the solvability relation for quasiorders (or even the definition of types for quasiorders) to circumvent this problem. The next proposition shows a limit to this approach.

Proposition 5.20. There is no congruence $\rho$ of Quo $\mathbf{S}$ such that $\left.\rho\right|_{\text {Con } \mathbf{S}}=\sim_{s}$, more precisely, the congruence of Quo $\mathbf{S}$ generated by the congruence solvability relation is $1_{\mathbf{S}}$.

Proof. Let $\sim$ be the congruence of Quo $\mathbf{S}$ generated by the congruence solvability relation. We will need the following elements of Quo $\mathbf{S}$ :

$$
\begin{aligned}
\bar{\tau} & =\{(0,1),(1,0)\} \\
\overline{\eta_{1}} & =\{(0,1),(0,2),(2,1),(3,1)\} \\
\overline{\eta_{2}} & =\{(0,1),(0,2),(2,1),(3,1),(3,2)\} \\
\overline{\eta_{3}} & =\{(0,1),(0,2),(0,3),(2,1)\} \\
\overline{\eta_{4}} & =\{(0,1),(0,2),(0,3),(2,1),(2,3)\} \\
\overline{\eta_{5}} & =\{(0,1),(3,2)\} \\
\overline{\eta_{6}} & =\{(0,1),(2,3)\} \\
\overline{\eta_{7}} & =\{(0,1),(0,2),(2,1)\}
\end{aligned}
$$


Of course, $\tau$ is a congruence such that $\left(0_{\mathbf{S}}, \tau\right)$ is a congruence prime quotient of type 1. Therefore, $0_{\mathbf{S}} \sim \tau$, so as $\eta_{1} \vee \tau=\eta_{2} \vee \tau$ and $\eta_{3} \vee \tau=\eta_{4} \vee \tau, \eta_{1} \sim \eta_{2}$ and $\eta_{3} \sim \eta_{4}$. Note that $\eta_{5}<\eta_{2}, \eta_{6}<\eta_{4}$, and $\eta_{1} \wedge \eta_{5}=\eta_{3} \wedge \eta_{6}=\eta_{5} \wedge \eta_{6}$. From these follows that $\eta_{5} \sim \eta_{5} \wedge \eta_{6} \sim \eta_{6}$.

By $\eta_{7}>\eta_{5} \wedge \eta_{6}$

$$
\eta_{7}=\eta_{7} \vee\left(\eta_{5} \wedge \eta_{6}\right) \sim \eta_{7} \vee \eta_{5} \vee \eta_{6} \sim \eta_{7} \vee \eta_{5} \vee \eta_{6} \vee \tau=1_{\mathbf{S}}
$$

But as $\sim_{s}$ is closed to inversion (meaning that $\delta_{1} \sim_{s} \delta_{2}$ implies $\delta_{1}^{-1} \sim_{s} \delta_{2}^{-1}$ ), $\sim$ must also be closed to inversion. Thus, $\eta_{7}^{-1} \sim 1_{\mathbf{S}}$, and $0_{\mathbf{S}}=\eta_{7} \wedge \eta_{7}^{-1} \sim 1_{\mathbf{S}}$, so $\sim$ is the full relation on Quo $\mathbf{S}$.

We note that the above proof did not use the symmetry of $\sim$, so the quasiorder of Quo $\mathbf{S}$ generated by the congruence solvability relation is also the full relation.

Problem 5.21. Is the solvability relation on the quasiorder lattices of algebras that omit 1 for quasiorders/for congruences a lattice congruence? What about varieties omitting 1 ?

An other difference for quasiorders is that Proposition 4.21 does not generalize for them: a counterexample is the two-element lattice, which is obviously a minimal algebra, yet its quasiorder lattice is the direct square of the twoelement lattice. A more elaborate counterexample: consider the semilattice $(\{0,1,2,3\}, \min )$. If $\bar{\alpha}=\{(0,1),(0,2),(1,2)\}$, and $\beta$ is the full order $<$, then the semilattice is $(\alpha, \beta)$-minimal, but the reader can easily check that the interval $[\alpha, \beta]$ is the four-element chain. However, there are no other kinds of counterexamples.

Theorem 5.22. Suppose $\mathbf{A}$ is $(\alpha, \beta)$-minimal, and $\alpha \nprec \beta$ in Quo A. Then the interval $[\alpha, \beta]$ either:

- contains only types 4 , and is isomorphic to the direct square of the twoelement lattice, or

- contains only types 5, and is a distributive lattice, or

- contains only types 1 and 2 .

Proof. First suppose that $\left(\gamma_{1}, \gamma_{2}\right)$ is a type 3 or a type 4 quasiorder prime quotient in the interval, and take an edge $(a, b) \in \gamma_{2} \backslash \gamma_{1}$. By Theorem 5.17, there are pseudo-meet operations for both $a$ and $b$, so by Lemma 5.13, all $\beta \backslash \alpha$ edges have $a$ and $b$ as either source ot target. Therefore, $\beta \backslash \alpha \subseteq\{(a, b),(b, a)\}$, and by $\alpha \nprec \beta$ there must be equality. Furthermore, either $\alpha \cup\{(a, b)\}$ or $\alpha \cup\{(b, a)\}$ must be a quasiorder.

We need yet to show that if either is a quasiorder, then both are. So suppose that $\delta_{1}:=\alpha \cup\{(a, b)\}$ is a quasiorder, and $\delta_{2}:=\alpha \cup\{(b, a)\}$ is not. Notice that $\delta_{2}$ is a preorder, as it is in the quasiorder $\beta$, and its transitive closure does not contain $(a, b)$, because there is no path from $a$ to $b$ containing only $\alpha$-edges 
and the edge $(b, a)$. This means that there is a unary polynomial $p$ such that $p\left(\delta_{2}\right) \notin \delta_{2}$. Any unary polynomial maps all $\alpha$-edges into $\delta_{1}$-edges, and $(b, a)$ into a $\beta$ edge, which means that $p$ maps the edge $(b, a)$ into $(a, b)$. But then it also maps $(a, b)$ into $(b, a)$, a contradiction.

So in this case, the interval $[\alpha, \beta]$ is isomorphic to the lattice $\mathbf{2}^{2}$. By Theorem 5.17 it only contains types 4 , because the quasiorders of any prime quotient in it differ only in a single edge $((a, b)$ or $(b, a))$, and there is a pseudo-meet-pseudojoin pair for that edge.

Now suppose that $\left(\gamma_{1}, \gamma_{2}\right)$ is a type 5 quasiorder prime quotient in the interval $[\alpha, \beta]$, and $(a, b) \in \gamma_{2} \backslash \gamma_{1}$. It can be assumed by Theorem 5.17 that there is a pseudo-meet operation for $a$, in which case by Lemma 5.13, all $\beta \backslash \alpha$ edges have $a$ as either source or target. So there are elements $x_{1}, \ldots, x_{k}, y_{1}, \ldots, y_{l} \in A$ such that

$$
\beta \backslash \alpha=\left\{\left(x_{1}, a\right), \ldots,\left(x_{k}, a\right),\left(a, y_{1}\right), \ldots,\left(a, y_{l}\right)\right\},
$$

with $k$ or $l$ possibly being zero.

Suppose there is an $i$ so that there is a pseudo-meet operation for $x_{i}$ (the case when there is one for $y_{i}$ is similar). This means that, by Lemma 5.13, all $\beta \backslash \alpha$ edges have $x_{i}$ as either source or target, so $k=1$, and either $l=0$ or $l=1$ and $y_{1}=x_{1}$. The first case is impossible by $\alpha \nprec \beta$, in the second, the quasiorders in any prime quotient in the interval $[\alpha, \beta]$ differ only in one edge, and there is a pseudo-meet-pseudo-join pair for it, which by Theorem 5.17 contradicts the assumption that this interval contains a type 5 .

Therefore, there is no pseudo-meet operation for any of the $x_{i}$ or the $y_{i}$. By Theorem 5.17, this means that the interval $[\alpha, \beta]$ contains only types 5 . To prove that this interval is a distributive lattice, it is enough to note that for quasiorders $\alpha \leq \delta_{1}, \delta_{2} \leq \beta, \delta_{1} \vee \delta_{2}=\delta_{1} \cup \delta_{2}$, because for any $1 \leq i \leq k$ and $1 \leq j \leq l$, the edge $\left(x_{i}, y_{j}\right)$ is in $\alpha$ (as it is in $\beta$ and $a$ is neither its target or source).

Problem 5.23. Is it true that for any finite distributive lattice $\mathbf{L}$, there is a finite algebra $\mathbf{A}$, and quasiorders $\alpha, \beta \in$ Quo $\mathbf{A}$ such that $\mathbf{A}$ is $(\alpha, \beta)$-minimal and the interval $[\alpha, \beta]$ of Quo $\mathbf{A}$ contains only types 5 and is isomorphic to $\mathbf{L}$ ?

About the third case, we can say a little more, if the interval omits type 1 , namely, that in that case the interval is a modular lattice. This is true for congruences without assuming minimality:

Theorem 5.24. ([21], Corollary 6.8) Suppose that $\alpha<\beta$ in Con A, and all the congruence types in the interval $N:=[\alpha, \beta]$ are 2. Then $N$ is a modular lattice.

Theorem 5.25. Suppose that $\alpha<\beta$ in Quo A, $\mathbf{A}$ is $(\alpha, \beta)$-minimal, and all the quasiorder types in the interval $N:=[\alpha, \beta]$ are 2. Then $N$ is a modular lattice.

Proof. By Proposition 5.8, $N$ contains only *-quotients. Consider the mapping $\delta \mapsto \delta^{*}$ from $N$ into Con $\mathbf{A}$. This is a $\wedge$-homomorphism, and preserves the $\prec$ relation by Proposition 5.2. This means that it is also injective. 


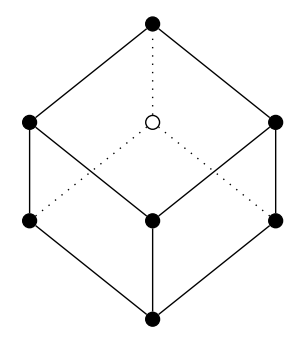

Figure 5.3: The inclusion mapping from $\mathbf{D}_{1}$ to $\mathbf{2}^{3}$ is an order-preserving $\wedge$ homomorphism, but not a $\vee$-homomorphism

Claim 1. For any $\delta_{1}, \delta_{2} \in N$,

$$
\delta_{1}^{*} \vee \delta_{2}^{*} \vee\left(\delta_{1} \wedge \delta_{2}\right)=\delta_{1} \vee \delta_{2}
$$

To see this, notice that the left side is in $N$, and

$$
\left(\delta_{1}^{*} \vee \delta_{2}^{*} \vee\left(\delta_{1} \wedge \delta_{2}\right)\right)^{*} \geq \delta_{1}^{*}
$$

thus, $\delta \mapsto \delta^{*}$ being order-preserving,

$$
\delta_{1}^{*} \vee \delta_{2}^{*} \vee\left(\delta_{1} \wedge \delta_{2}\right) \geq \delta_{1}
$$

The same is true for $\delta_{2}$ instead of $\delta_{1}$, which concludes the non-trivial direction of the equality. The claim is proved.

Claim 2. $\delta \mapsto \delta^{*}$ is also a $\vee$-homomorphism.

Suppose contrariwise that $\delta_{1}^{*} \vee \delta_{2}^{*}<\left(\delta_{1} \vee \delta_{2}\right)^{*}$ in Con A. Since $\delta \mapsto \delta^{*}$ preserves $\prec$, there is a chain $\alpha^{*}=\gamma_{0} \prec \gamma_{1} \prec \cdots \prec \gamma_{2}=\beta^{*}$ in Con A such that $\operatorname{typ}\left(\gamma_{i}, \gamma_{i+1}\right)=2$ for all $i$. By Lemma 4.24, any $\alpha^{*}$-class can be mapped to any other $\alpha^{*}$-class in the same $\beta^{*}$-class by a bijective unary polynomial. Therefore, any $\delta_{1}^{*} \vee \delta_{2}^{*}$-class can be mapped to any other $\delta_{1}^{*} \vee \delta_{2}^{*}$-class in the same $\left(\delta_{1} \vee \delta_{2}\right)^{*}$-class by a bijective unary polynomial.

We call a $\delta_{1} \wedge \delta_{2}$ edge crossedge if it is between different $\delta_{1}^{*} \vee \delta_{2}^{*}$-classes of the same $\left(\delta_{1} \vee \delta_{2}\right)^{*}$-class. Take a pair $(a, b) \in\left(\delta_{1} \vee \delta_{2}\right)^{*} \backslash\left(\delta_{1}^{*} \vee \delta_{2}^{*}\right)$. By Claim 1 , there is a $\left(\delta_{1}^{*} \vee \delta_{2}^{*}\right)-\left(\delta_{1} \wedge \delta_{2}\right)$ path from $a$ to $b$. This path must contain at least one crossedge (all edges in this path are in $\delta_{1} \vee \delta_{2}$, and $(a, b)$ is in $\left(\delta_{1} \vee \delta_{2}\right)^{*}$, so all edges in this path are in $\left.\left(\delta_{1} \vee \delta_{2}\right)^{*}\right)$.

Hence, there exists a crossedge. Its source can be mapped by a bijective polynomial into any other $\delta_{1}^{*} \vee \delta_{2}^{*}$-class of its $\left(\delta_{1} \vee \delta_{2}\right)^{*}$-class, so from any $\delta_{1}^{*} \vee \delta_{2}^{*}$ class of that $\left(\delta_{1} \vee \delta_{2}\right)^{*}$-class there is a crossedge. Therefore, from any element of that $\left(\delta_{1} \vee \delta_{2}\right)^{*}$-class there is a crossedge, consequently, there is a circle of crossedges. This contradicts $\left(\delta_{1} \wedge \delta_{2}\right)^{*} \leq \delta_{1}^{*} \vee \delta_{2}^{*}$. The claim is proved.

Thus $\delta \mapsto \delta^{*}$ must map any sublattice of $N$ isomorphic to $\mathbf{N}_{5}$ to a sublattice of the interval $\left[\alpha^{*}, \beta^{*}\right]$ of Con $\mathbf{A}$ isomorphic to $\mathbf{N}_{5}$, and for any two comparable elements of this sublattice there must be a chain of type 2 quotients (in Con $\mathbf{A}$ ). 
By Lemma 6.5 of [21], there is no such sublattice of Con $\mathbf{A}$ (the condition there holds because the sublattice would have to lie in a solvability block-see Theorem 4.18).

Note: It is not generally true for (finite) lattices that a $\prec$-preserving $\wedge$ homomorphism is also a lattice homomorphism. As seen on Figure 5.3, omitting the element $(1,0,1)$ from the distributive lattice $\mathbf{2}^{3}$ yields a lattice isomorphic to the lattice $\mathbf{D}_{1}$. The inclusion map from this latter lattice to $\mathbf{2}^{3}$ is a $\wedge$ homomorphism, preserves $\prec$, but it is not a $\vee$-homomorphism, as $(1,0,0) \vee$ $(0,0,1)$ is $(1,0,1)$ in the distributive lattice, and $(1,1,1)$ in the other.

Problem 5.26. Is Theorem 5.24 true for quasiorders? If not, is it true if the algebra generates a variety omitting 1 ? 


\section{Chapter 6}

\section{Varieties omitting certain types}

When discussing varieties omitting certain types, there is no distinction between congruence and quasiorder types:

Theorem 6.1. For any $i \in\{1,2,3,4,5\}$ and any variety $\mathcal{V}, \mathcal{V}$ omits $i$ for congruences iff it omits $i$ for quasiorders.

Proof. Suppose that $\mathbf{A}$ is a finite algebra in $\mathcal{V},(\alpha, \beta)$ is a prime quasiorder quotient of $\mathbf{A}$ with type $i$. If $(\alpha, \beta)$ is a ${ }^{*}$-quotient, then $\operatorname{typ}\left(\alpha^{*}, \beta^{*}\right)=i$, so $\mathcal{V}$ does not omit $i$ for congruences. i Now assume that $(\alpha, \beta)$ is a +-quotient. By definition, its type is determined by types of congruence quotients in the enlargement $\mathbf{M}_{+}$of an $(\alpha, \beta)$-minimal set $M$ (for the sake of simplicity, we use the notation $\left.\mathbf{M}=\left.\mathbf{A}\right|_{M}\right)$.

By Theorem 4.11, there is an idempotent unary polynomial $e$ mapping $A$ onto $M$ such that $e(\beta) \nsubseteq \nsubseteq \alpha$. If $e(x)=t\left(x, a_{1}, \ldots, a_{k}\right)$, where $t$ is a term of $\mathbf{A}$ and $a_{1}, \ldots, a_{k} \in A$, we define $e^{\prime}(x):=t\left(x,\left(a_{1}, a_{1}, a_{1}\right), \ldots,\left(a_{k}, a_{k}, a_{k}\right)\right)$, which is an idempotent unary polynomial mapping $\mathbf{A}_{+}$onto $\mathbf{M}_{+}$. (Both $\mathbf{A}_{+}$and $\mathbf{M}_{+}$ are meant as enlargements by $\beta$.)

Consider the restriction mapping from Con $\mathbf{A}_{+}$to $\operatorname{Con} \mathbf{M}_{+}$. As $M_{+}=$ $e^{\prime}\left(A_{+}\right)$, this mapping is onto by Proposition 4.3, more precisely, for any congruence $\mu \in$ Con $\mathbf{M}_{+}$, the restriction of the congruence $\mu_{0} \in$ Con $\mathbf{A}_{+}$generated by $\mu$ to $M_{+}$is $\mu$. Therefore, for any prime quotient $(\mu, \nu)$ of Con $\mathbf{M}_{+}$, there are congruences $\mu_{0} \leq \mu_{1} \prec \nu_{1} \leq \nu_{0}$ of Con $\mathbf{A}_{+}$such that the restriction of $\mu_{1}$ and $\nu_{1}$ to $M_{+}$is $\mu$ and $\nu$, respectively. But then, a minimal $\left(\mu_{1}, \nu_{1}\right)$-set of $\mathbf{A}_{+}$ contained in $M_{+}$(there is such a minimal set, for $M_{+}$is a polynomial image of $\left.A_{+}\right)$is also a $(\mu, \nu)$-minimal set of $\mathbf{M}_{+}$. (Note that all polynomials of $\mathbf{M}_{+}$can be obtained as restrictions of polynomials of $\mathbf{A}_{+}$.) Thus $\operatorname{typ}\left(\mu_{1}, \nu_{1}\right)=\operatorname{typ}(\mu, \nu)$, so each type in the congruence lattice of $\mathbf{M}_{+}$(including $\operatorname{typ}(\alpha, \beta)$ ) is present at the congruence lattice of $\mathbf{A}_{+}$. As $\mathbf{A}_{+} \in \mathcal{V}, i$ is not omitted by $\mathcal{V}$ for congruences in this case, either. 
In contrast to the preceding theorem, it is not true that any algebra omits the same congruence and quasiorder types. For example, take the algebra $\mathbf{B}=$ $(\{0,1,2\},\{p, q\})$, where $p$ and $q$ are binary operations with multiplication tables given below:

\begin{tabular}{l|lll}
$p$ & 0 & 1 & 2 \\
\hline 0 & 0 & 0 & 0 \\
1 & 0 & 1 & 1 \\
2 & 0 & 1 & 2
\end{tabular}

\begin{tabular}{c|ccc}
$q$ & 0 & 1 & 2 \\
\hline 0 & 0 & 0 & 0 \\
1 & 0 & 1 & 2 \\
2 & 2 & 2 & 2
\end{tabular}

This is a simple algebra, $\left(0_{\mathbf{B}}, 1_{\mathbf{B}}\right)$ has (congruence) type 5 , and with $\delta=$ $\{(0,0),(0,1),(0,2),(1,1),(2,2)\}$ and $\leq=\{(0,0),(0,1),(0,2),(1,1),(1,2),(2,2)\}$, the quasiorder type of $\left(0_{\mathrm{B}}, \delta\right)$ is 5 and the type of $(\delta, \leq)$ is 4 . (It is easy to check that $\delta$ and $\leq$ are indeed quasiorders.)

Problem 6.2. Which combinations of congruence and quasiorder omitting types are possible in a finite algebra?

\subsection{Congruence modular varieties}

Lemma 6.3. Suppose that $\mathbf{A}$ is a finite algebra in a congruence modular variety that is $(\alpha, \beta)$-minimal for quasiorders $\alpha<\beta$, where $\alpha^{*}=\beta^{*}$. Then $\beta^{*}$ has exactly two blocks.

Proof. As $\beta$ is not a congruence, there are elements $a, b \in A$ such that $a /$ $\beta^{*} \prec_{\beta / \beta^{*}} b / \beta^{*}$ and $(a, b) \notin \alpha$. As $\mathbf{A}$ is in a congruence modular variety, it admits Gumm terms $p_{1}, \ldots, p_{n}, q$ (see Theorem 4.27).

For each $1 \leq i \leq n$,

$$
a=p_{i}(a, a, a) \stackrel{\beta}{\rightarrow} p_{i}(a, a, b) \stackrel{\beta}{\rightarrow} p_{i}(a, b, b) \stackrel{\beta}{\rightarrow} p_{i}(b, b, b)=b,
$$

so both $p_{i}(a, a, b)$ and $p_{i}(a, b, b)$ is in the $\beta^{*}$-block of either $a$ or $b$. Notice that there must be a $j$ so that $p_{j}(a, a, b)$ and $p_{j}(a, b, b)$ are in different $\beta^{*}$-blocks, otherwise by the Gumm identities $p_{n}(a, b, b)$ would be in the $\beta^{*}$-block of $a$, which contradicts $b=q(a, a, b) \stackrel{\beta}{\rightarrow} q(a, b, b)=p_{n}(a, b, b)$.

The unary polynomial $p_{j}(a, x, b)$ thus maps $(a, b)$ to a $\beta \backslash \alpha$-edge (as $\alpha^{*}=\beta^{*}$, any edge with source in the $\beta^{*}$-block of $a$ and target in the $\beta^{*}$-block of $b$ must not be in $\alpha)$. By the $(\alpha, \beta)$-minimality of $\mathbf{A}, p_{j}(a, x, b)$ must be a bijective polynomial, but as

$$
a=p_{j}(a, x, a) \stackrel{\beta}{\rightarrow} p_{j}(a, x, b) \stackrel{\beta}{\rightarrow} p_{j}(b, x, b)=b,
$$

this polynomial maps $A$ to the union of the $\beta^{*}$-blocks of $a$ and $b$. Thus $\beta^{*}$ only has two blocks.

Lemma 6.4. Let $\mathbf{A}$ be a finite algebra in a congruence modular variety. If $\mathbf{A}$ is minimal to a quasiorder quotient, then it either is a two-element algebra, or has a Mal'tsev polynomial. 
Proof. By Lemma 4.8, A is minimal to either a congruence quotient or a quasiorder +-quotient $(\alpha, \beta)$. In the second case, by Lemma $6.3, \beta^{*}$ has two blocks. This is only possible if $\alpha=\beta^{*}$, but then by Lemma $4.6, \mathbf{A}$ is also $\left(\alpha, 1_{\mathbf{A}}\right)$ minimal.

Hence, $\mathbf{A}$ is necessarily minimal to a congruence quotient, and obviously, it must then be minimal to a congruence prime quotient. By Theorem 4.27, that quotient has either type 2,3 , or 4 , and has no tail. In the type 2 case, the pseudoMal'tsev term for this quotient (see Proposition 4.22) must be a Mal'tsev-term, in the type 3 or 4 case the body of $\mathbf{A}$ (with respect to this congruence quotient) equals $A$, and must be two-element by Proposition 4.20 .

As a use of this lemma, we will generalize (only for locally finite varieties) Theorem 2 of [5], which states that in a congruence modular variety, the transitive closure of the intersection of two tolerances coincides with the intersection of the transitive closures.

Theorem 6.5. Let $\mathbf{A}$ an algebra in a locally finite congruence modular variety, and denote with $\bar{\delta}$ the transitive closure of a compatible reflexive relation $\delta$ on $A$. The equality $\bar{\rho} \cap \bar{\sigma}=\bar{\rho} \cap \bar{\sigma}$ is satisfied for arbitrary reflexive compatible relations $\rho, \sigma$ of $\mathbf{A}$. Thus taking transitive closures induces a homomorphism from the lattice of compatible reflexive relations of $\mathbf{A}$ to Quo $\mathbf{A}$.

Proof. Suppose $\overline{\rho \cap \sigma}<\bar{\rho} \cap \bar{\sigma}$. It can be assumed that $\mathbf{A}$ is finite, as if $(a, b)$ is an element of the right side and not of the left, there are elements of $A$ $c_{1}, \ldots, c_{k}, d_{1}, \ldots, d_{l}$ such that $a \stackrel{\rho}{\rightarrow} c_{1} \stackrel{\rho}{\rightarrow} \ldots \stackrel{\rho}{\rightarrow} c_{k} \stackrel{\rho}{\rightarrow} b$ and $a \stackrel{\sigma}{\rightarrow} d_{1} \stackrel{\sigma}{\rightarrow} \ldots \stackrel{\sigma}{\rightarrow}$ $d_{l} \stackrel{\sigma}{\rightarrow} b$, and the elements $a, b, c_{1}, \ldots, c_{k}, d_{1}, \ldots, d_{l}$ generate a finite counterexample.

Take a $\nu \in$ Quo $\mathbf{A}$ so that $\overline{\rho \cap \sigma} \prec \nu \leq \bar{\rho} \cap \bar{\sigma}$. It can be assumed that $\mathbf{A}$ is a $(\overline{\rho \cap \sigma}, \nu)$-minimal algebra, because otherwise, its restriction to a minimal set will yield a counterexample of smaller cardinality.

By Lemma 6.4, $\mathbf{A}$ is either a two-element algebra, or has a Mal'tsev polynomial. The first case is impossible: it is very easy to see that this theorem does not have a two-element counterexample. In the second case, all the reflexive compatible relations of $\mathbf{A}$ are tolerances: By Theorem 2 of [5] (what this theorem generalizes), this is a contradiction.

In [18], the author and his advisor proved the following theorem:

Theorem 6.6. If a locally finite variety is congruence distributive, then it is also quasiorder distributive. If it is congruence modular, then it is also quasiorder modular.

We used directed Jónsson and directed Gumm terms (see [23]) to prove this theorem. These satisfy equations that are a modifications of the equations satisfied by the usual Jónsson and Gumm terms, and define equivalent Mal'tsev classes (so, for example the existence of Day terms and directed Day terms in an algebra are equivalent). With the use of Lemma 6.4, this theorem can be generalized: it is true not only for distributivity and modularity, but any identity between them. 
Theorem 6.7. Suppose that $\mathbf{A}$ is a finite algebra in a congruence modular variety. Then $\mathbf{C o n} \mathbf{A}$ and $\mathbf{Q u o} \mathbf{A}$ satisfy the same lattice identities.

Proof. Obviously, any identity satisfied by Quo $\mathbf{A}$ is also satisfied by Con $\mathbf{A}$. Suppose that the converse is not true, that there is a lattice identity $p \approx q$ that holds in Con A, and does not hold in Quo A. We will assume two things. Firstly, that $p \leq q$ is an identity that holds in all lattices (and so $p \approx q$ is equivalent to $p \nless q$ ). Secondly, that $\mathbf{A}$ is a minimal counterexample, in the sense that for every $\mathbf{B}$ with smaller cardinality, if $\mathbf{B}$ lies in a congruence modular variety, and Con $\mathbf{B}$ satisfies $p \approx q$, then Quo $\mathbf{B}$ also satisfies $p \approx q$.

The fact that $p \approx q$ is not satisfied by Quo $\mathbf{A}$ means that there are quasiorders $\alpha_{1}, \ldots, \alpha_{n}, \mu, \nu$ of $\mathbf{A}$ such that

$$
p\left(\alpha_{1}, \ldots, \alpha_{n}\right) \leq \mu \prec \nu \leq q\left(\alpha_{1}, \ldots, \alpha_{n}\right)
$$

holds in Quo A ( $p$ and $q$ are assumed to be $n$-ary). For a $(\mu, \nu)$-minimal set $U$, the algebra $\left.\mathbf{A}\right|_{U}$ is in a congruence modular variety (Proposition 4.26 and Theorem 4.27), Quo $\left.\mathbf{A}\right|_{U}$ does not satisfy $p \approx q$, but Con $\left.\mathbf{A}\right|_{U}$ does (because it is a homomorphic image of Con $\mathbf{A}$ by Proposition 4.3 and Theorem 4.11).

Therefore, by the minimality assumption, $\mathbf{A}$ must be $(\mu, \nu)$-minimal. By Lemma 6.4, it is either a two-element algebra or has a Mal'tsev polynomial. Both are impossible. In the first case the congruence lattice of the algebra is isomorphic to the two-element lattice, and the quasiorder lattice is isomorphic either to the same, or to its direct square, so they satisfy the same identities. In the second case, Quo $\mathbf{A}=$ Con $\mathbf{A}$ by Proposition 2.24.

Corollary 6.8. Suppose that $\mathcal{P}$ is a lattice identity so that each variety whose congruence lattices satisfy $\mathcal{P}$ is congruence modular. Then if all congruence lattices of a locally finite variety satisfy $\mathcal{P}$, then so do all the quasiorder lattices of the variety.

We note that the condition here for $\mathcal{P}$ is weaker then the condition that it should be a stronger lattice identity than modularity. For example, the so-called Arguesian identity is a weaker lattice identity than modularity, but a variety is congruence Arguesian precisely if it is congruence modular (see [22]).

Problem 6.9. For which lattice identities is it true that if the congruence lattices of a locally finite variety satisfy it, then so do the quasiorder lattices of the variety? Does the answer change without assuming locally finiteness? In particular, is it true that for any lattice identity stronger than modularity, if the congruence lattices of the variety satisfy it then so do the quasiorder lattices?

Problem 6.10. Is Corollary 6.8 true for quasi-identities?

Problem 6.11. Is there a general way of obtaining Quo $\mathbf{A}$ from Con $\mathbf{A}$ for a finite $\mathbf{A}$ in a congruence modular variety using the H, S, P operators? (According to 6.7 , they are in the same lattice variety. The answer to the this problem is given in [7] for lattices: the quasiorder lattice of a lattice is isomorphic the direct square of the congreuence lattice.) 
In Chapter 5 we posed the question whether the solvability relation is a congruence on the quasiorder lattice for varieties omitting type 1 . While we think that the slightly more likely answer to this is "no", we will prove that for (finite) algebras in congruence modular varieties, it is indeed a congruence. This is a purely lattice-theoretic consequence of Theorem 5.18.

We cannot use the same notation for quasiorder and congruence solvability, because we do not know whether the former is an extension of the latter.

Definition 6.12. For quasiorders $\alpha, \beta$ of the finite algebra $\mathbf{A}$, the pair $(\alpha, \beta)$ is in the solvability relation of Quo $\mathbf{A}$ (denoted by $\alpha \sim_{q s} \beta$ ) if the interval $[\alpha \wedge \beta, \alpha \vee \beta]$ of Quo A omits types 3, 4 and 5. (As Proposition 5.20 showed, $\sim_{q s}$ is generally not a lattice congruence.)

The following proposition is evident from Dedekind's modularity condition.

Proposition 6.13. If $a \prec b$ in a modular lattice $\mathbf{L}$, then $a \wedge c \preceq b \wedge c$ and $a \vee c \preceq b \vee c$ hold for every $c \in L$, and there cannot be equality at both.

Theorem 6.14. Suppose that $\mathbf{A}$ is a finite algebra and Quo $\mathbf{A}$ is a modular lattice. Then the solvability relation of Quo $\mathbf{A}$ is a lattice congruence.

Proof. We will prove the compatibility of $\sim_{q s}$ first. Suppose that $\alpha \sim_{q s} \beta$, and take any quasiorder $\delta$, we will show that $\alpha \wedge \delta \sim_{q s} \beta \wedge \delta$. It can be assumed that $\alpha<\beta$, because $(\alpha \wedge \beta) \wedge \delta \sim_{q s}(\alpha \vee \beta) \wedge \delta$ is a stronger statement than $\alpha \wedge \delta \sim_{q s} \beta \wedge \delta$

Suppose that $\alpha \wedge \delta \leq \mu \prec \nu \leq \beta \wedge \delta$ with $\operatorname{typ}(\mu, \nu) \notin\{1,2\}$. As $\mu \wedge \alpha=$ $\nu \wedge \alpha=\alpha \wedge \delta$, it follows from Proposition 6.13 that $\mu \vee \alpha \prec \nu \vee \alpha$. As $\mu \vee \alpha$ and $\nu \vee \alpha$ are in the interval $[\alpha, \beta], \operatorname{typ}(\mu \vee \alpha, \nu \vee \alpha) \in\{1,2\}$. This is a contradiction: $(\mu, \nu)$ and $(\mu \vee \alpha, \nu \vee \alpha)$ are prime perspective quotients, so by Theorem 5.18, they have the same type.

A dual reasoning shows that the join with any given quasiorder also preserves $\sim_{q s}$, and as $\sim_{q s}$ is reflexive, it is compatible.

We still need to prove that $\sim_{q s}$ is transitive: take quasiorders such that $\alpha \sim_{q s} \beta \sim_{q s} \gamma$, and suppose that there is a prime quotient $(\mu, \nu)$ in the interval $[\alpha \wedge \gamma, \alpha \vee \gamma]$ that has type other than 1 and 2. By Proposition 6.13, either $\mu \wedge \beta \prec \nu \wedge \beta$ or $\mu \vee \beta \prec \nu \vee \beta$, we will assume that the first holds of the two (the other case is similar).

Now, again by Proposition 6.13, either $(\mu \wedge \beta) \vee \alpha \prec(\nu \wedge \beta) \vee \alpha$, or $\mu \wedge \beta \wedge \alpha \prec$ $\nu \wedge \beta \wedge \alpha$. In the second case, as $\mu \wedge \beta \wedge \alpha \wedge \gamma=\nu \wedge \beta \wedge \alpha \wedge \gamma$, another application of Proposition 6.13 shows that $(\mu \wedge \beta \wedge \alpha) \vee \gamma \prec(\nu \wedge \beta \wedge \alpha) \vee \gamma$. In either case, there is a quotient that is prime projective to $(\mu, \nu)$ in one of the intervals $[\alpha \wedge \beta, \alpha \vee \beta]$ or $[\beta \wedge \gamma, \beta \vee \gamma]$, which contradicts $\alpha \sim_{q s} \beta \sim_{q s} \gamma$ by Theorem 5.18 .

Quasiorder solvability for algebras in congruence modular varieties has additional properties.

Theorem 6.15. If $\mathbf{A}$ is a finite algebra in a congruence modular variety, then Quo $\mathbf{A} / \sim_{q s}$ is a distributive lattice. 
Proof. We need to show that $(\alpha \wedge \gamma) \vee(\beta \wedge \gamma) \sim_{q s}(\alpha \vee \beta) \wedge \gamma$ for any $\alpha, \beta, \gamma \in$ Quo A. Assume that this is not true, with $\mathbf{A}$ being a counterexample of minimal cardinality. There are quasiorders $\mu$ and $\nu$ with

$$
(\alpha \wedge \gamma) \vee(\beta \wedge \gamma) \leq \mu \prec \nu \leq(\alpha \vee \beta) \wedge \gamma
$$

and $\operatorname{typ}(\mu, \nu) \neq 2$. Take a $(\mu, \nu)$-minimal set $U$, the algebra $\left.\mathbf{A}\right|_{U}$ is in a congruence modular variety by Proposition 4.26 and Theorem 4.27. As by Propositions 4.3 and 4.11 ,

$$
\left(\left.\left.\alpha\right|_{U} \wedge \gamma\right|_{U}\right) \vee\left(\left.\left.\beta\right|_{U} \wedge \gamma\right|_{U}\right) \leq\left.\left.\mu\right|_{U} \prec \nu\right|_{U} \leq\left.\left(\left.\left.\alpha\right|_{U} \vee \beta\right|_{U}\right) \wedge \gamma\right|_{U}
$$

and $\operatorname{typ}\left(\left.\mu\right|_{U},\left.\nu\right|_{U}\right)=\operatorname{typ}(\mu, \nu)$ by the definitions of types, $\left.\mathbf{A}\right|_{U}$ is a counterexample. Hence by the assumption of minimal cardinality, $\mathbf{A}$ must be $(\mu, \nu)$-minimal.

According to Lemma 6.4, A has either only two elements, or has a Mal'tsev polynomial. The first case is impossible because a two-element algebra has a quasiorder lattice that is isomorphic to either $\mathbf{2}$ or $\mathbf{2}^{2}$, and both are distributive. In the second case, Quo $\mathbf{A}=$ Con $\mathbf{A}$, and clearly $\sim_{q s}=\sim_{s}$, so Con $\mathbf{A} / \sim_{s}$ should be a nondistributive lattice. But that cannot be, because it is an upper bounded lattice by Theorem 4.18, and obviously it is also modular.

Lemma 6.16. Suppose that $\mathbf{A}$ is a finite algebra in a congruence modular variety. If the interval $[\mu, \nu]$ of Con $\mathbf{A}$ contains only type 2 prime quotients, then the same interval in Quo A also only contains type 2 prime quotients.

Proof. Suppose that the triple $(\mathbf{A}, \mu, \nu)$ is a counterexample such that the $|A|$ is minimal, and the interval $[\mu, \nu]$ is also minimal (with respect to inclusion). There is a quasiorder prime quotient $(\alpha, \beta)$ in this interval such that $\operatorname{typ}(\alpha, \beta) \neq 2$. The assumption for $[\mu, \nu]$ means that $\mu=\alpha^{*}$ and $\nu=\beta \vee \beta^{-1}$.

$\mathbf{A}$ is $(\alpha, \beta)$-minimal, otherwise restricting to an $(\alpha, \beta)$-minimal set would yield a smaller counterexample (see a more detailed explanation of this idea in the proof of Theorem 6.15, for example).

It follows from Proposition 6.13 that either $\left(\alpha \wedge \alpha^{-1}, \beta \wedge \alpha^{-1}\right)$ or $\left(\alpha \vee \alpha^{-1}, \beta \vee\right.$ $\left.\alpha^{-1}\right)$ is a prime quotient. Whichever is prime quotient, it is prime perspective to $(\alpha, \beta)$, therefore it has type other than 2 by Theorem 5.18 , and is in the interval $[\mu, \nu]$. In the second case, this means by the minimality assumption for $[\mu, \nu]$ that $\alpha \vee \alpha^{-1}=\mu$, consequently, $\alpha=\mu$. We can also assume $\alpha=\mu$ in the first case, because then the quotient $(\alpha, \beta)$ can be exchanged to $\left(\mu, \beta \wedge \alpha^{-1}\right)$.

So from now on $\alpha=\mu$, which means by Lemma 4.6 that $\mathbf{A}$ is $(\mu, \nu)$-minimal. Obviously, $\mu \nprec \nu$ in Quo A, so Theorem 5.22 can be used. As A is in a congruence modular variety, which omits types 1 and 5 by Theorem 4.27, and $[\mu, \nu]$ contains a quasiorder type that is not 2 , the only possibility is that this interval (of Quo A) is isomorphic to $\mathbf{2}^{2}$, and contains only types 4. By Theorem 5.17, $\beta \backslash \alpha$ is a single edge, and there is a pseudo-meet-pseudo-join pair for it. That edge is also a $\nu \backslash \mu$ edge. But as the congruence type of $(\mu, \nu)$ is 2 , and $\mathbf{A}$ is $(\mu, \nu)$-minimal, there cannot be a pseudo-meet operation for a $\nu \backslash \mu$ edge. 
Theorem 6.17. Suppose that $\mathbf{A}$ is a finite algebra in a congruence modular variety. Then the solvability relation of $\mathbf{Q u o} \mathbf{A}$ is the lattice congruence generated by the congruence solvability relation.

Proof. Lemma 6.16 shows that $\sim_{s}$ is contained in the restriction of $\sim_{q s}$ to Con $\mathbf{A}$, so $\sim_{s}$ generates a congruence of Quo $\mathbf{A}$ not larger than $\sim_{q s}$. For the other direction, take a quasiorder prime quotient $(\alpha, \beta)$ of type 2. By Proposition 5.8, it is a *-quotient, and by the definition of types of *-quotients, $\alpha^{*} \prec \beta^{*}$ in $\operatorname{Con} \mathbf{A}$, and $\operatorname{typ}\left(\alpha^{*}, \beta^{*}\right)=2$. As $(\alpha, \beta)=\left(\alpha^{*} \vee \alpha, \beta^{*} \vee \alpha\right)$, and $\alpha^{*} \sim_{s} \beta^{*}$, $(\alpha, \beta)$ is in the congruence of Quo $\mathbf{A}$ generated by $\sim_{s}$. As $\sim_{q s}$ is the congruence generated by the type 2 quasiorder quotients, indeed $\sim_{q s}$ is in the congruence of Quo $\mathbf{A}$ generated by $\sim_{s}$.

These theorems show that solvability generalizes quite well for quasiorders in congruence modular varieties. A concept strongly related to solvability is the commutator, a binary operator on the congruence lattice of a (not necessarily finite) algebra (see [26]).

Definition 6.18. The commutator of the congruences $\alpha, \beta \in$ Con $\mathbf{A}$ (denoted by $\alpha \star \beta$ ) is the smallest congruence $\delta$ such that for any $t \in \operatorname{Term}_{k} \mathbf{A}$ and for any $a, b, c_{2}, \ldots, c_{k}, d_{2}, \ldots, d_{k} \in A$ satisfying $(a, b) \in \alpha,\left(c_{i}, d_{i}\right) \in \beta$ for all $2 \leq i \leq k$,

$$
\left(t\left(a, c_{2}, \ldots, c_{k}\right), t\left(a, d_{2}, \ldots, d_{k}\right)\right) \in \delta \Leftrightarrow\left(t\left(b, c_{2}, \ldots, c_{k}\right), t\left(b, d_{2}, \ldots, d_{k}\right)\right) \in \delta
$$

holds.

We avoided the usual notation for the commutator- $[\alpha, \beta]$-because we used that frequently for lattice intervals, especially in this section.

Nothing in this definition uses symmetry, so there is no problem of extending it for quasiorders. Unfortunately, this definition does not seem to be meaningful. While for congruences $\alpha \star \beta \leq \alpha \wedge \beta$, moreover, the commutator is commutative for congruence modular varieties, for quasiorders neither are/ true. To see this, consider the variety of lattices with 0 and 1 . The natural order $\leq$ of a lattice is a quasiorder. For any quasiorder $\beta$, and any pair of lattice elements $(c, d) \in \beta$, it follows from the certainly true $0 \wedge c \stackrel{\leq \star \beta}{\longrightarrow} 0 \wedge d$ and $(0,1) \in \alpha$ that $1 \wedge c \stackrel{\leq \star \beta}{\longrightarrow} 1 \wedge d$. Thus, $(c, d) \in \beta$ implies $(c, d) \in \leq \star \beta$, so $\beta=\leq \star \beta$ (the other inclusion follows from the definition).

Problem 6.19. What are the properties of the commutator for quasiorders, particularly in the congruence modular case? What is its relation to the solvability relation? Is there a way to extend the commutator on the congruence lattice (in a meaningful way) so that $\alpha \star \beta \leq \alpha \wedge \beta$ and/or $\alpha \star \beta=\beta \star \alpha$ remain true?

\subsection{Varieties omitting types 1 and 2}

Theorem 6.20. Suppose $\mathbf{A}$ is finite, and generates a variety that omits 1. If $\mathbf{M}$ is a simple sublattice of Quo $\mathbf{A}$, then it is a modular lattice. If $\operatorname{HSP}(\mathbf{A})$ also omits 2, then $\mathbf{M}$ must be a trivial or a two-element lattice. 
Proof. It can be assumed that $\mathbf{A}$ is $\left(0_{M}, 1_{M}\right)$-minimal, because if $0_{M} \leq \gamma_{1} \prec$ $\gamma_{2} \leq 1_{M}$ in Quo $\mathbf{A}$, and $U$ is a $\left(\gamma_{1}, \gamma_{2}\right)$-minimal set, then $\mathbf{A} \mid U$ generates a variety omitting 1 , and its quasiorder lattice contains a sublattice isomorphic to a nontrivial homomorphic image of $\mathbf{M}$. (Because $\left.\delta \mapsto \delta\right|_{U}$ is a lattice homomorphism from Quo A to Quo $\left.\mathbf{A}\right|_{U}$ which does not collapse $\gamma_{1}$ and $\gamma_{2}$, and $\mathbf{M}$ is simple.)

By Theorem 5.22, the interval $\left[0_{M}, 1_{M}\right]$ is either a distributive lattice, or it only contains types 2 . In the latter case, the interval is modular by Theorem 5.25 , thus $M$ is also modular as its sublattice. If the variety omits 2 as well as 1 , then $M$ is a simple distributive lattice, hence it is a two-element lattice.

As an immediate consequence, Theorem 9.10. of [21] can be extended. (See Theorem 6.22. there for the implication $(4) \Rightarrow(3)$.)

Corollary 6.21. For a locally finite variety $\mathcal{V}$, the following are equivalent.

1. $\mathcal{V}$ omits 1 and 2 ,

2. $\mathcal{V}$ is congruence meet semidistributive,

3. no congruence lattice of algebras in $\mathcal{V}$ contains a sublattice isomorphic to $\mathbf{M}_{3}$,

4. no congruence lattice of finite algebras in $\mathcal{V}$ contains a sublattice isomorphic to $\mathbf{M}_{3}$,

5. no quasiorder lattice of finite algebras in $\mathcal{V}$ contains a sublattice isomorphic to $\mathbf{M}_{3}$,

We do not know such an extension of Theorem 9.6. describing locally finite varieties omitting 1 .

Corollary 6.21 feels lacking, as only one of the three equivalent congruence conditions has its quasiorder analogue there. To see that the analogues of the other two are not equivalent to these conditions, it is sufficient to study the quasiorder lattices of semilattices. The variety of semilattices is the most basic example for a congruence meet semidistributive lattice (it obviously only contains type 5). It is also clearly locally finite. The class of congruence lattices of semilattices is quite rich, satisfying no nontrivial lattice identities ([11]).

Theorem 6.22. The quasiorder lattice of $\mathrm{FS}(3)$ (the 3-generated free semilattice) is not a meet semidistributive lattice.

Proof. Let $X=\{a, b, c\}$. Take $\alpha_{0}, \beta_{0}$, and $\gamma$ as the quasiorders of $\operatorname{FS}(X)$ generated respectively by $(a, b),(b, c)$, and $(a, c)$. Both $\left(\gamma \wedge \alpha_{0}\right) \backslash \beta_{0}$ and $(\gamma \wedge$ $\left.\beta_{0}\right) \backslash \alpha_{0}$ contain only a single edge, namely, $(a \wedge b \wedge c, b \wedge c)$, and $(a \wedge b, a \wedge b \wedge c)$, respectively. Therefore, with $\alpha=\operatorname{Tr}\left(\alpha_{0} \cup\{(a \wedge b, a \wedge b \wedge c)\}\right)$, and $\beta=\operatorname{Tr}\left(\beta_{0} \cup\right.$ $\{(a \wedge b \wedge c, b \wedge c)\})$,

$$
\alpha \wedge \gamma=\beta \wedge \gamma<(\alpha \vee \beta) \wedge \gamma,
$$

the inequality holding because only the right side contains $(a, c)$. Note that $\alpha$ and $\beta$ are quasiorders: both $(a \wedge b \wedge c, b \wedge c)$, and $(a \wedge b, a \wedge b \wedge c)$ are mapped into 


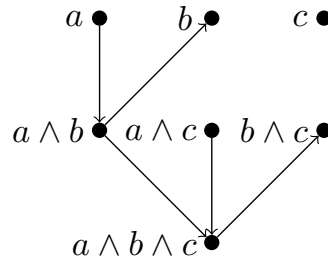

$\alpha$

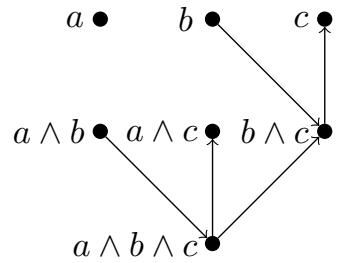

$\beta$

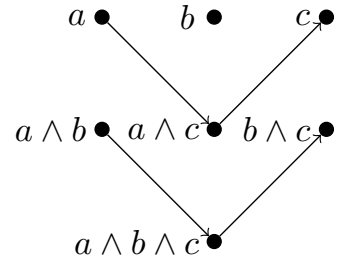

$\gamma$

Figure 6.1: The quasiorders $\alpha, \beta, \gamma$ of $\operatorname{FS}(3)$ satisfying $\alpha \wedge \gamma=\beta \wedge \gamma<(\alpha \vee \beta) \wedge \gamma$

themselves or a loop by any unary polynomial, so $\alpha_{0} \cup\{(a \wedge b, a \wedge b \wedge c)\}$ and $\beta_{0} \cup\{(a \wedge b \wedge c, b \wedge c)\}$ are compatible reflexive relations, hence their transitive closures are quasiorders. Thus $\operatorname{Quo}(\mathrm{FS}(X))$ fails meet semi-distributivity.

A little more can be said. A characterization of a locally finite variety omitting 1 (according to the aforementioned Theorem 9.6. of [21]) is that the congruence lattices of the variety are meet semidistributive over modular, that is, each has a congruence such that all of its classes are modular, and the factor with it is a meet semidistributive lattice. As that congruence (for finite algebras of the variety, at least) is the solvability relation, which is trivial for semilattices, one does not expect this to generalize for quasiorders. Indeed, it does not.

Theorem 6.23. Quo(FS(5)) (the 5-generated free semilattice) is not a meet semidistributive over modular lattice.

Proof. It will be enough to repeat the argument used in the proof of Theorem 6.22 with a modification: we essentially double everything so that the interval $[\alpha \wedge \gamma, \gamma]$ will be large enough to not be modular.

Let $X=\{a, b, c, d, e\}$. Take $\alpha_{0}, \beta_{0}$, and $\gamma$ as the quasiorders of $\operatorname{FS}(X)$ generated respectively by $\{(a, b),(c, d)\},\{(b, c),(d, e)\}$, and $\{(a, c),(c, e)\}$. The quasiorders $\alpha$ and $\beta$ are gained by adding the $\beta_{0} \wedge \gamma$-edges to $\alpha_{0}$, and the $\alpha_{0} \wedge \gamma$-edges to $\beta_{0}$, respectively, and taking transitive closures. It can be checked manually that

$$
\alpha \wedge \gamma=\beta \wedge \gamma<\gamma=(\alpha \vee \beta) \wedge \gamma,
$$

and the interval $[\alpha \wedge \gamma, \gamma]$ of $\operatorname{Quo}(\operatorname{FS}(X))$ contains a sublattice isomorphic to $\mathbf{N}_{5}$. (Add first the edge $(a, c),(a \wedge b, b \wedge e)$, and $(a \wedge c, a \wedge c \wedge e)$, second, the edges $(a, c)$ and $(a, e)$, finally, the edge $(c, e)$ to $\alpha \wedge \gamma$, and generate quasiorders. These three quasiorders generate a lattice isomorphic to $\mathbf{N}_{5}$.)

Now any congruence of $\operatorname{Quo}(\mathrm{FS}(X))$ with a meet semidistributive factor must contain the interval $[\alpha \wedge \gamma, \gamma]$ in the same class, and therefore that class cannot be modular. The proof is finished.

The remainder of this section is concerned about quasiorders of infinite semilattices. We begin with giving an example of a lattice of posets on an infinite 

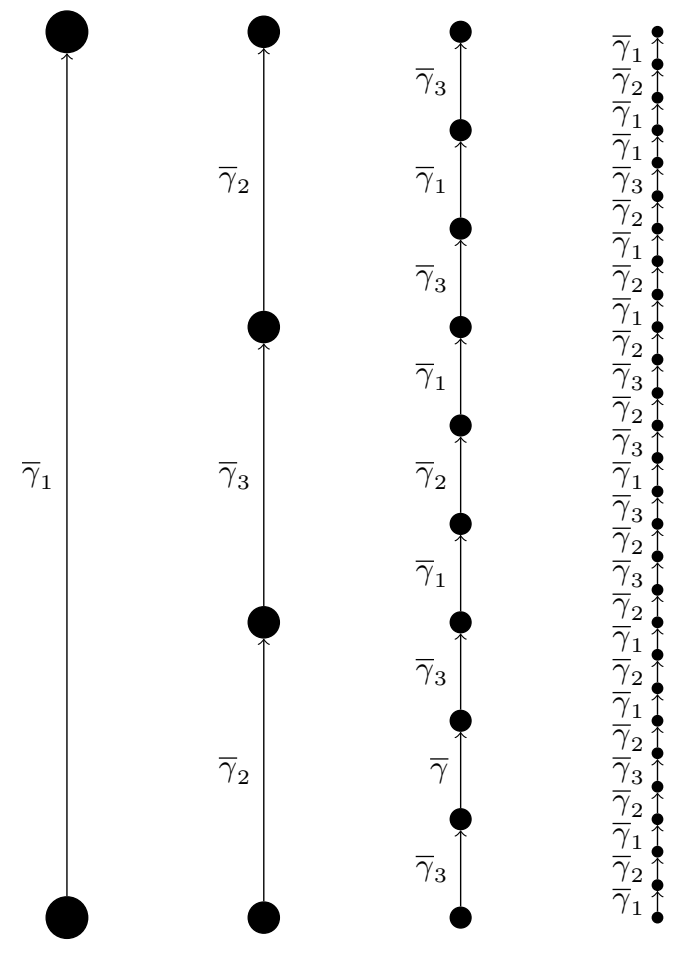

Figure 6.2: The core edges of the $\bar{\gamma}_{i}$

set isomorphic to $\mathbf{M}_{3}$. Note that by Theorem 3.2 this cannot be done on a finite set.

Example 6.24. Take $C$ as the set of finite ternary fractions in the interval $[0,1]$.

We define on $C$ the preorders $\bar{\gamma}_{1}, \bar{\gamma}_{2}, \bar{\gamma}_{3}$ by giving a generating set for each (to which we will refer to as their cores). The set of the core edges consists of all the pairs of the form $\left(\frac{a}{3^{j}}, \frac{a+1}{3^{j}}\right)$, where $a, j$ are integers, $0 \leq j$ and $0 \leq a \leq 3^{j}-1$. A core edge is called an up, a neutral, or a down edge, depending on whether the remainder of $a$ modulo 3 is 0,1 , or 2 .

The allocation of the core edges to exactly one of the $\bar{\gamma}_{i}$ will be done recursively. The pair $(0,1)$ is in the core of $\bar{\gamma}_{1}$. Assume that $\left(\frac{a}{3^{j}}, \frac{a+1}{3^{j}}\right)$ is in the core of $\bar{\gamma}_{i}$. Then $\left(\frac{3 a}{3^{j+1}}, \frac{3 a+1}{3^{j+1}}\right)$ and $\left(\frac{3 a+2}{3^{j+1}}, \frac{3 a+3}{3^{j+1}}\right)$ will be in the core of $\bar{\gamma}_{i+1}$, while $\left(\frac{3 a+1}{3^{j+1}}, \frac{3 a+2}{3^{j+1}}\right)$ will be in the core of $\bar{\gamma}_{i-1}$.

In the above example and henceforth until the end of this section, the index $i$ is meant to be modulo 3 .

Lemma 6.25. In Example 6.24, the sublattice generated by $\bar{\gamma}_{1}, \bar{\gamma}_{2}$ and $\bar{\gamma}_{3}$ in the lattice of preorders of $C$ contains only partial orders, and it is isomorphic to $\mathbf{M}_{3}$. 
Proof. It is enough to show that the intersection of any two of the $\bar{\gamma}_{i}$ is $0_{C}$, as by $\bar{\gamma}_{i} \subseteq \bar{\gamma}_{i+1} \circ \bar{\gamma}_{i-1} \circ \bar{\gamma}_{i+1}$ the join of any two of them is the natural full order $\leq$ of $C$.

We have to prove that if $x<y$, and there is a path of core $\bar{\gamma}_{i}$-edges from $x$ to $y$, then there is no path of core $\bar{\gamma}_{i+1}$-edges from $x$ to $y$. Suppose the contrary. Notice that in a path of core edges (of a given $\bar{\gamma}_{i}$ ) there is at most one neutral edge, which must precede all the up edges, and must be preceded by all the down edges. If there is no neutral edge, any down edge must precede any up edge.

Suppose that $\frac{a}{3^{j}} \leq x<y \leq \frac{a+1}{3^{j}}$ (where $j$ is minimal).

If there are only up edges in the core $\bar{\gamma}_{i}$-path from $x$ to $y$, then the length of any edge of this path is at most the third of the length of the preceding edge. This means that the first edge is $\left(\frac{3 a}{3^{j+1}}, \frac{3 a+1}{3^{j+1}}\right)$. Any neutral or down core $\bar{\gamma}_{i+1}$-edge from $\frac{3 a}{3^{j+1}}$ is at least $\frac{1}{3^{j}}$ long, which is longer than the length of the $\bar{\gamma}_{i}$-path. Hence the $\bar{\gamma}_{i+1}$-path must contain only up edges. This is impossible: the first edge of this path is either at least thrice, or at most third the length of the first edge of the $\bar{\gamma}_{i}$-path. In the first case the length of the $\bar{\gamma}_{i+1}$-edge is at least twice the length of the $\bar{\gamma}_{i}$-edge, in the second case at most half of it.

Thus, the path of core $\bar{\gamma}_{i}$-edges, and likewise, the path of core $\bar{\gamma}_{i+1}$-edges, must start with either a neutral or a down edge. But there is either a single neutral or a single down edge starting at any element of $C$ (besides 1 ), and that cannot be a core edge of both $\bar{\gamma}_{i}$ and $\bar{\gamma}_{i+1}$.

Now, we inject Example 6.24 into a semilattice, namely, $\mathrm{FS}(C)$. All elements of $\operatorname{FS}(C)$ are intersections of finitely many elements of $C$, we will refer to those elements as the factors of the given semilattice element (so, for example, $0 \wedge 1$ is an element of $\operatorname{FS}(C)$ having two factors: 0 and 1).

Note that the $\bar{\gamma}_{i}$ are binary relations of this semilattice, but they are not quasiorders (not even preorders, as reflexivity fails). In order to get a sublattice in $\mathrm{Quo}(\mathrm{FS}(C))$ isomorphic to $\mathbf{M}_{3}$ we will use a similar construction as in the proof of Theorem 6.22: from preorders of the free generator, we take the quasiorders generated by them, then add any edge that is in two of them to the third. The difference is that this time, this construction does not yield quasiorders, and so we repeat this step (generating quasiorders, then adding edges so their pairwise intersections coincide) infinitely, then take the limit.

So, denote the quasiorders of $\operatorname{FS}(C)$ generated by $\bar{\gamma}_{i}$ with $\gamma_{i}^{(0)}$. Fortunately, these quasiorders are easily understandable (the proof of the following lemma is trivial):

Lemma 6.26. Let $X$ be a set, $\bar{\delta} \in \operatorname{Quo}(X)$, and $\delta^{(0)}$ the quasiorder of $F S(X)$ generated by $\bar{\delta}$. Then for $c_{1}, \ldots, c_{m}, d_{1}, \ldots, d_{n} \in X, c_{1} \wedge \cdots \wedge c_{m} \stackrel{\delta^{(0)}}{\longrightarrow} d_{1} \wedge \cdots \wedge d_{n}$ holds iff for each $1 \leq i \leq m$ and each $1 \leq j \leq n$ there are $i^{\prime}, j^{\prime}$ such that $c_{i} \stackrel{\bar{\delta}}{\rightarrow} d_{j^{\prime}}$ and $c_{i^{\prime}} \stackrel{\bar{\delta}}{\rightarrow} d_{j}$.

Any two of the $\gamma_{i}^{(0)}$ generate the same quasiorder, but their pairwise intersections do not coincide. This is because by the previous lemma, for any 
$a, b, c, d \in C$ satisfying

$$
a \stackrel{\bar{\gamma}_{1}}{\longrightarrow} b \stackrel{\bar{\gamma}_{2}}{\longleftarrow} c \stackrel{\bar{\gamma}_{1}}{\longrightarrow} d
$$

the edge $(a \wedge c \wedge d, a \wedge b \wedge d)$ is in $\gamma_{1}^{(0)} \wedge \gamma_{2}^{(0)}$. It is easy to choose such elements of $C$ :

$$
0 \stackrel{\bar{\gamma}_{1}}{\longrightarrow} 1=\frac{3}{3} \stackrel{\bar{\gamma}_{2}}{\longleftarrow} \frac{2}{3}=\frac{18}{27} \stackrel{\bar{\gamma}_{1}}{\longrightarrow} \frac{19}{27} .
$$

As neither 0,1 , or $\frac{19}{27}$ is above $\frac{2}{3}$ in the quasiorder $\bar{\gamma}_{3}$,

$$
\left(0 \wedge \frac{2}{3} \wedge \frac{19}{27}, 0 \wedge 1 \wedge \frac{19}{27}\right) \in\left(\gamma_{1}^{(0)} \wedge \gamma_{2}^{(0)}\right) \backslash \gamma_{3}^{(0)}
$$

Set recursively for $k>0$

$$
\gamma_{i}^{(k)}=\gamma_{i}^{(0)} \vee\left(\gamma_{i-1}^{(k-1)} \wedge \gamma_{i+1}^{(k-1)}\right)
$$

it is immediate by induction that for each $i$ these form an ascending chain. Let $\gamma_{i}$ be their union. The goal is to prove that the $\gamma_{i}$ generate a sublattice of $\mathrm{Quo}(\mathrm{FS}(C))$ isomorphic to $\mathbf{M}_{3}$. The easier part is the following lemma.

Lemma 6.27. The pairwise meets of the $\gamma_{i}$-s coincide, as do their pairwise joins.

Proof. For any element $e \in \gamma_{i-1} \wedge \gamma_{i+1}$, there is a $k$ such that $e \in \gamma_{i-1}^{(k)}$ and $e \in \gamma_{i+1}^{(k)}$, hence $e \in \gamma_{i}^{(k+1)} \subseteq \gamma_{i}$.

For joins, notice that as the pairwise joins of the $\bar{\gamma}$ coincide, so do the pairwise joins of the $\gamma_{i}^{(0)}$, denote this join with $\nu$. (Actually, $\nu$ is the quasiorder of $\operatorname{FS}(C)$ generated by the full order $<$ on $C$ ). Clearly, for any $i$ and any $k, \gamma_{i}^{(k)} \leq \nu$, thus $\gamma_{i-1} \vee \gamma_{i+1} \leq \nu$. As $\gamma_{i} \geq \gamma_{i}^{(0)}, \gamma_{i-1} \vee \gamma_{i+1} \geq \nu$ also holds for each $i$.

We need yet to show that the $\gamma_{i}$-s do not coincide. The way the $\gamma_{i}$-s were obtained from the $\gamma_{i}^{(0)}$-s is a little more complicated then the way the $\gamma_{i}^{(0)}$ were obtained from the $\bar{\gamma}_{i}$. We need a vocabulary to deal with them, hence the need for the following (atrociously long) definition.

Definition 6.28. For a pair $u, v \in \mathrm{FS}(C)$, a nonnegative integer $s$ and $i \in$ $\{1,2,3\}$, we say that a pair $(K, d)$, where $K$ is a finite set and $d$ is a mapping $d: K \rightarrow\{0,1, \ldots, s\}$ (called the height function), is a diagram verifying $u \stackrel{\gamma_{i}^{(s)}}{\longrightarrow}$ $v$, if the following hold:

- The elements of $K$ are elements of $\operatorname{FS}(C)$ indexed by a nonnegative integer (essentially, $K$ is a subset of $\operatorname{FS}(C)$, with certain elements possibly appearing multiple times),

- there is a natural $m$ such that there are elements $k_{0}, \ldots, k_{m} \in K$ such that:

$-k_{0}=u, k_{m}=v$, and $k_{0}, \ldots, k_{m}$ are precisely the elements of $K$ whose height is $s$, 


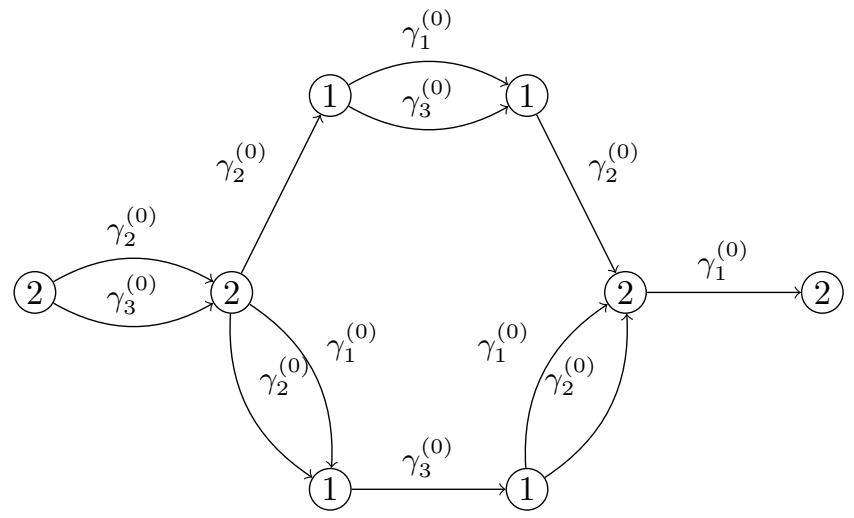

Figure 6.3: Diagram example

- for any $0 \leq j<m$, either $k_{j} \stackrel{\gamma_{i}^{(0)}}{\longrightarrow} k_{j+1}$, or there are subsets $L_{+}, L_{-} \subseteq K$ such that the pairs $\left(L_{+}, d_{+}\right)$and $\left(L_{-}, d_{-}\right)$are diagrams respectively verifying $k_{j} \stackrel{\gamma_{i+1}^{(s-1)}}{\longrightarrow} k_{j+1}$ and $k_{j} \stackrel{\gamma_{i-1}^{(s-1)}}{\longrightarrow} k_{j+1}$, where $d_{+}$ and $d_{-}$are just the restrictions of $d$ to $L_{+}$and $L_{-}$with the exception that $d_{+}\left(k_{j}\right), d_{+}\left(k_{j+1}\right), d_{-}\left(k_{j}\right)$ and $d_{-}\left(k_{j+1}\right)$ equal $s-1$ instead of $s$ (such diagrams are called subdiagrams),

- for any proper subset $K^{\prime}$ of $K$, the pair $\left(K^{\prime},\left.d\right|_{K^{\prime}}\right)$ does not satisfy the preceding property.

It is immediate from the definition that two elements of $\operatorname{FS}(C)$ are in $\gamma_{i}^{(s)}$ iff there is a diagram verifying it. We make the following observations of diagrams.

Lemma 6.29. For a diagram $(K, d)$ verifying $u \stackrel{\gamma_{i}^{(s)}}{\longrightarrow} v$, there is both a $\gamma_{i}^{(0)} \cup \gamma_{i+1}^{(0)}$ path and $a \gamma_{i}^{(0)} \cup \gamma_{i-1}^{(0)}$ path from $k_{j_{1}}$ to $k_{j_{2}}$ in $K$ for any $0 \leq j_{1}<j_{2} \leq m$ (so specifically also from $u$ to $v$ ).

Proof. We use induction on $s$. It is enough to prove that there are such paths from $k_{j}$ to $k_{j+1}$ for all $0 \leq j<m$. If $k_{j} \stackrel{\gamma_{i}^{(0)}}{\longrightarrow} k_{j+1}$, this is immediate. Otherwise, there are subdiagrams of $(K, d)$ verifying both $k_{j} \stackrel{\gamma_{i-1}^{(s-1)}}{\longrightarrow} k_{j+1}$ and $k_{j} \stackrel{\gamma_{i+1}^{(s-1)}}{\longrightarrow}$ $k_{j+1}$, and by using the inductive assumption the needed paths are gained.

Lemma 6.30. Suppose $u, v \in \mathrm{FS}(C)$, and $(K, d)$ a diagram verifying $u \stackrel{\gamma_{i}^{(s)}}{\longrightarrow} v$. Take the denominators of all the factors of the elements of $K$ (written so the nominators and denominators are coprime). The largest of these denominators appears as a denominator of one of the factors of $u$ or $v$. 
Proof. We use induction on $s$, without fixing $i$. Define (for all $i$ ) $\gamma_{i}^{(-1)}$ as the equality relation on $\operatorname{FS}(C)$. Note that this is in accordance with $\gamma_{i}^{(k)}=$ $\gamma_{i}^{(0)} \vee\left(\gamma_{i-1}^{(k-1)} \wedge \gamma_{i+1}^{(k-1)}\right)$.

Now the lemma is obvious for $s=-1$. For $s>-1$, an element of $K$ containing a factor with a maximal denominator either equals a $k_{j}$ for some $0 \leq j \leq m$, or is in a subdiagram verifying $k_{j^{\prime}} \stackrel{\gamma_{i^{\prime}}^{(s-1)}}{\longrightarrow} k_{j^{\prime}+1}$ for some $0 \leq j^{\prime}<m$ and $i^{\prime} \in\{1,2,3\}$. By the inductive assumption, in the latter case there also is a $k_{j}$ having a factor with a maximal denominator.

Suppose that $k_{j}=c_{1} \wedge \cdots \wedge c_{t}$, where $c_{1}=\frac{b}{3^{r}}$, with $3^{r}$ being the largest denominator appearing among the factors of the elements of $K$, and $b$ not being divisible by 3 . We will assume that the remainder of $b$ modulo 3 is 1 (the other case is similar, only it yields that $u$ has a factor with denominator $3^{r}$ ). Thus $\left(\frac{b}{3^{r}}, \frac{b+1}{3^{r}}\right)$ is a neutral core edge of $\bar{\gamma}_{i^{\prime \prime}}$ for an $i^{\prime \prime} \in\{1,2,3\}$.

By Lemma 6.29, there is both a $\gamma_{i}^{(0)} \cup \gamma_{i-1}^{(0)}$ path and a $\gamma_{i}^{(0)} \cup \gamma_{i+1}^{(0)}$ path from $k_{j}$ to $v$ in $K$.

Suppose first that $i$ differs from $i^{\prime \prime}$, in this case we will consider the first of these paths.

By Lemma 6.26, the path

$$
k_{j}=d_{0} \stackrel{\gamma_{i}^{(0)}}{\longrightarrow} d_{1} \stackrel{\gamma_{i-1}^{(0)}}{\longrightarrow} d_{2} \stackrel{\gamma_{i}^{(0)}}{\longrightarrow} \ldots \stackrel{\gamma_{i-1}^{(0)}}{\longrightarrow} d_{h}=v
$$

in $K$ implies a path

$$
\frac{b}{3^{r}}=e_{0} \stackrel{\bar{\gamma}_{i}}{\longrightarrow} e_{1} \stackrel{\bar{\gamma}_{i-1}}{\longrightarrow} e_{2} \stackrel{\bar{\gamma}_{i}}{\longrightarrow} \ldots \stackrel{\bar{\gamma}_{i-1}}{\longrightarrow} e_{h}
$$

in $C$, with $e_{t}$ being a factor of $d_{t}$ for all $0 \leq t \leq h$.

There is no $\bar{\gamma}_{i}$ edge, and at most one $\bar{\gamma}_{i-1}$ edge from $\frac{b}{3^{r}}$ whose target has denominator not greater than $3^{r}$ : the edge $\left(\frac{b}{3^{r}}, \frac{b+1}{3^{r}}\right)$. If this is indeed a $\bar{\gamma}_{i-1}$ edge (this is so in the $i^{\prime}=i-1$ case), then $\left(\frac{b+1}{3^{r}}, \frac{b+2}{3^{r}}\right)$ is a (down) $\bar{\gamma}_{i+1}$ edge, which means that there is no $\bar{\gamma}_{i}$ or $\bar{\gamma}_{i-1}$ edge from $\frac{b+1}{3^{r}}$ with a target whose denominator is not greater than $3^{r}$. Therefore, all the $e_{t}$ are equal to $\frac{b}{3^{r}}$ or $\frac{b+1}{3^{r}}$ (those equal to the first preceding in the above path those equal to the second).

Now suppose that $i=i^{\prime \prime}$, and consider the $\gamma_{i}^{(0)} \cup \gamma_{i+1}^{(0)}$ path. As in the first case, we have the paths

$$
k_{j}=d_{0} \stackrel{\gamma_{i}^{(0)}}{\longrightarrow} d_{1} \stackrel{\gamma_{i+1}^{(0)}}{\longrightarrow} d_{2} \stackrel{\gamma_{i}^{(0)}}{\longrightarrow} \ldots \stackrel{\gamma_{i+1}^{(0)}}{\longrightarrow} d_{h}=v
$$

and

$$
\frac{b}{3^{r}}=e_{0} \stackrel{\bar{\gamma}_{i}}{\longrightarrow} e_{1} \stackrel{\bar{\gamma}_{i+1}}{\longrightarrow} e_{2} \stackrel{\bar{\gamma}_{i}}{\longrightarrow} \ldots \stackrel{\bar{\gamma}_{i+1}}{\longrightarrow} e_{h},
$$

with $e_{t}$ being a factor of $d_{t}$ for all $0 \leq t \leq h$. Now $\left(\frac{b}{3^{r}}, \frac{b+1}{3^{r}}\right)$ is a $\bar{\gamma}_{i}$ edge, but $\left(\frac{b+1}{3^{r}}, \frac{b+2}{3^{r}}\right)$ is neither a $\bar{\gamma}_{i}$ or $\bar{\gamma}_{i+1}$ edge, and again all the $e_{t}$ are equal to either $\frac{b}{3^{r}}$ or $\frac{b+1}{3^{r}}$.

As $e_{h}$ is a factor of $v$, and (in both cases) equals either $\frac{b}{3^{r}}$ or $\frac{b+1}{3^{r}}$ where the remainder of $b$ modulo 3 is 1 , the proof is finished. 
Lemma 6.31. $\gamma_{1} \neq \gamma_{2}$.

Proof. Suppose $0 \stackrel{\gamma_{2}}{\longrightarrow} 1$. There must be a diagram verifying it, and by Lemma 6.30 that diagram can only contain elements of $\operatorname{FS}(C)$ that have only factors with denominator 1 . Such a diagram can only contain 0,1 , or $0 \wedge 1$, and it is obvious that no such diagram exists.

With the preceding lemma, we have proved the following theorem.

Theorem 6.32. Quo(FS $(\omega)$ ) contains a sublattice isomorphic to $\mathbf{M}_{3}$.

$M_{3}$ was of interest to show that the third point of Corollary 6.21 does not extend into quasiorders. But it is natural to ask whether Theorem 6.32 is true for other lattices.

Conjecture 6.33. Any lattice is embeddable into the quasiorder lattice of a suitable semilattice.

We give here a method that, for any lattice $\mathbf{L}$, obtains a semilattice $\mathbf{S}$ and a homomorphism $\mathbf{L} \mapsto$ Quo $\mathbf{S}$ that we believe-but cannot prove-to be one-to-one. It is a modification of the method used in the proof of Theorem 6.32.

- Take a set $X_{0}$ and for any $l \in L$ preorders $\gamma_{l}^{(0, *)} \in \operatorname{Pre} X_{0}$ such that $l \mapsto \gamma_{l}^{(0, *)}$ is an embedding. This can be done by Theorem 3.1.

- Take $\mathbf{S}_{0}$ as the semilattice freely generated by $X_{0}$, and let $\gamma_{l}^{(0,0)}$ be the quasiorder of $\mathbf{S}_{0}$ generated by $\gamma_{l}^{(0, *)}$. Now $l \mapsto \gamma_{l}^{(0,0)}$ is a join homomorphism.

- Set recursively for $k>0$

$$
\gamma_{l}^{(0, k)}:=\gamma_{l}^{(0, k-1)} \vee \bigvee_{l_{1} \wedge l_{2} \leq l}\left(\gamma_{l_{1}}^{(0, k-1)} \wedge \gamma_{l_{2}}^{(0, k-1)}\right),
$$

and

$$
\gamma^{(0)}:=\bigcup \gamma_{l}^{(0, k)}
$$

Now $l \mapsto \gamma_{l}^{(0)}$ is a meet homomorphism. Unfortunately, it is not necessarily a join homomorphism. It was so for $M_{3}$. That was because the only nontrivial joins for $M_{3}$ are all 1 , and $\gamma_{1}^{(0,0)}$ and $\gamma_{1}^{(0)}$ coincides. On the other hand, the reader can check that for Figure 6.4,

$$
\gamma_{r_{1}}^{(0)} \vee \gamma_{r_{2}}^{(0)}=\gamma_{r_{1}}^{(0,0)} \vee \gamma_{r_{2}}^{(0,0)}=\gamma_{r}^{(0,0)}<\gamma_{r}^{(0)}
$$

- $X_{1}$ will be a set that is gained by adding an element $x_{y_{1}, y_{2}, r, r_{1}, r_{2}}$ to the set $S_{0}$ for any tuple $\left(y_{1}, y_{2}, r, r_{1}, r_{2}\right) \in S_{0}^{2} \times L^{3}$ such that

$$
\left(y_{1}, y_{2}\right) \in \gamma_{r}^{(0)} \backslash \gamma_{r_{1}}^{(0)} \vee \gamma_{r_{2}}^{(0)} .
$$




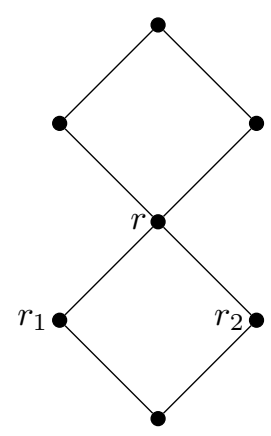

Figure 6.4: A lattice such that $l \mapsto \gamma_{l}^{(0)}$ is not a lattice homomorphism

- $\mathbf{S}_{1}$ is the semilattice that is presented by the generating set $X_{1}$ with all the identities that are true in $\mathbf{S}_{0}$. The preorders $\gamma_{l}^{(1, *)} \in \operatorname{Pre} S_{1}$ are obtained by adding all edges of the form $\left(y_{1}, x_{y_{1}, y_{2}, r, l, r_{2}}\right)$ and $\left(x_{y_{1}, y_{2}, r, r_{1}, l}, y_{2}\right)$ to $\gamma_{\bar{l}}^{(0)}$ for all $\bar{l} \geq l$.

- Define $\gamma_{l}^{(1,0)}$ as the quasiorder of $\mathbf{S}_{1}$ generated by $\gamma_{l}^{(1, *)}$. (Now, again, $l \mapsto \gamma_{l}^{(1,0)}$ is a join homomorphism!) From this, define $\left\{\gamma_{l}^{(1)}: l \in L\right\}$ the same way as $\left\{\gamma_{l}^{(0)}: l \in L\right\}$ was defined from $\left\{\gamma_{l}^{(0,0)}: l \in L\right\}$.

- Continuing in this fashion, for all natural $k$ the set $X_{k}$, the semilattice $\mathbf{S}_{k}$, and (for all $l \in L$ ) quasiorders $\gamma_{l}^{(k, 0)}<\gamma_{l}^{(k)}$ are defined, so that $l \mapsto \gamma_{l}^{(k, 0)}$ is a join homomorphism, and $l \mapsto \gamma_{l}^{(k)}$ is a meet homomorphism for any $k$. Furthermore, $\gamma_{l}^{(k)}$ is $\gamma_{l}^{(k+1,0)}$ constricted to $S_{k}$.

- Define $\mathbf{S}$ as the direct union of the $\mathbf{S}_{k}$, and $\gamma_{l}$ as the direct union of the $\gamma_{l}^{(k)}$. Now $l \mapsto \gamma_{l}$ is a lattice homomophism.

\subsection{Varieties omitting 1,2 , and 5}

The aim of this section is to generalize Freese, Kearnes, and Nation's result [12] stating that in a variety omitting 1,2, and 5, the congruence lattices of the finite algebras are not only $\vee$-semidistributive (Theorem 4.29), but lower bounded. (This is part of Corollary 27 of [12]. We do not know whether the other part-that these lattices are also upper bounded-is true for quasiorders. Theorem 23-the main theorem-is not, for two reasons: first, as Proposition 5.19 shows, one cannot even generally talk about Quo A/ $\sim_{s}$, second, according to Theorem 6.22, the quasiorder lattices of semilattices are not necessarily upper bounded, and the solvability relation for semilattices is the equality relation.)

Before we can prove lower boundedness, we first need to prove $\vee$-semidistributivity. 


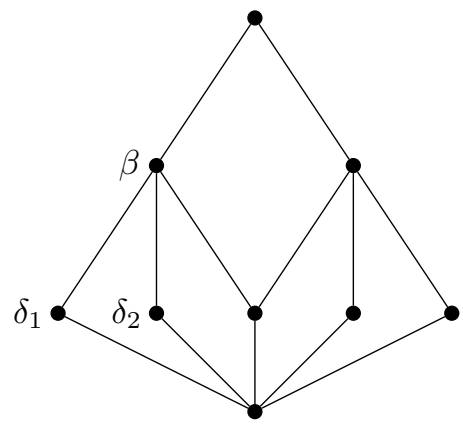

Figure 6.5: An involutive lattice not embeddable into a lattice of preorders, the involution being vertical reflection

Lemma 6.34. Suppose that $C$ is an arbitrary set, $\mathbf{L} \leq \operatorname{Pre} C$ is a lattice of preorders on $C$, and $\beta \in C$. Then the mapping $\delta \mapsto \delta \wedge \beta^{-1}$ is a lattice homomorphism from the ideal $(\beta]$ of $\mathbf{L}$ to the ideal $\left(\beta^{*}\right]$.

Proof. It is obvious that this mapping preserves meets. We need to show that for any $\delta_{1}, \delta_{2} \leq \beta$ :

$$
\left(\delta_{1} \vee \delta_{2}\right) \wedge \beta^{-1} \subseteq\left(\delta_{1} \wedge \beta^{-1}\right) \vee\left(\delta_{2} \wedge \beta^{-1}\right) .
$$

Consider an edge $(a, b)$ from the left side. There must be elements of $C a=$ $c_{0}, c_{1}, \ldots, c_{k}=b$ so that for each $0 \leq i<k,\left(c_{1}, c_{i+1}\right)$ is in either $\delta_{1}$ or $\delta_{2}$. As

$$
a=c_{0} \stackrel{\beta}{\rightarrow} c_{1} \stackrel{\beta}{\rightarrow} \ldots \stackrel{\beta}{\rightarrow} c_{k}=b \stackrel{\beta}{\rightarrow} a,
$$

all the $c_{i}$ are in the same $\beta^{*}$-block, so each $\left(c_{i}, c_{i+1}\right)$ is in either $\delta_{1} \wedge \beta^{-1}$ or $\delta_{2} \wedge \beta^{-1}$.

Corollary 6.35. For any set $C$, the following involutive lattice identity holds in Pre $C$ :

$$
\left(\left(\delta_{1} \wedge \beta\right) \vee\left(\delta_{2} \wedge \beta\right)\right) \wedge \beta^{-1}=\left(\delta_{1} \wedge \beta \wedge \beta^{-1}\right) \vee\left(\delta_{2} \wedge \beta \wedge \beta^{-1}\right)
$$

The above involutive lattice identity is nontrivial, as the Figure 6.5 shows. We note that Czédli in [6] gave a nontrivial involutive lattice quasi-identity that is satisfied in any preorder lattices. It is an interesting question whether there is a finite set of involutive lattice identities that characterizes preorder lattices, or at least the variety generated by them-but this question is outside of the scope of this thesis.

Theorem 6.36. Suppose that $\mathbf{A}$ is a finite algebra in a variety omitting 1, 2, and 5. Then Quo $\mathbf{A}$ is join semidistributive. 
Proof. Suppose that $\alpha, \gamma_{1}, \gamma_{2} \in$ Quo A are such that $\alpha \vee \gamma_{1}=\alpha \vee \gamma_{2}>\alpha \vee\left(\gamma_{1} \wedge\right.$ $\left.\gamma_{2}\right)$. It can be assumed that $\alpha=\alpha \vee\left(\gamma_{1} \wedge \gamma_{2}\right)$, and that $\beta:=\alpha \vee \gamma_{1} \succ \alpha$. Suppose that $\mathbf{A}$ is such that $|A|$ is minimal among counterexamples to the theorem, and the quasiorders are such that $\beta$ is minimal.

It follows from the minimal cardinality of $\mathbf{A}$ that $\mathbf{A}$ is $(\alpha, \beta)$-minimal, because for any $(\alpha, \beta)$-minimal set $U$, the algebra $\left.\mathbf{A}\right|_{U}$ is in a variety omitting 1 , 2, and 5 (see Proposition 4.26 and Theorem 4.29), and

$$
\left.\left.\alpha\right|_{U} \vee \gamma_{1}\right|_{U}=\left.\left.\alpha\right|_{U} \vee \gamma_{2}\right|_{U}=\left.\beta\right|_{U}>\left.\alpha\right|_{U}=\left.\alpha\right|_{U} \vee\left(\left.\left.\gamma_{1}\right|_{U} \wedge \gamma_{2}\right|_{U}\right)
$$

holds by Proposition 4.3 and Theorem 4.11.

We will consider two cases depending on whether $(\alpha, \beta)$ is a ${ }^{*}$-quotient or a +-quotient.

If $\alpha^{*} \neq \beta^{*}, \beta$ must be a congruence, otherwise intersecting $\alpha, \gamma_{1}, \gamma_{2}$ with $\beta^{-1}$ would yield a counterexample contradicting the minimality of $\beta$ (see Lemma $6.34)$. As $\mathbf{A}$ is $(\alpha, \beta)$-minimal, it also is $\left(\alpha^{*}, \beta\right)$-minimal. By Proposition 4.21, $\left(\alpha^{*}, \beta\right)$ is a prime congruence quotient. As typ $\left(\alpha^{*}, \beta\right) \in\{3,4\}$, there is a unique two-element $\left(\alpha^{*}, \beta\right)$-body (say, $\left.\{u, v\}\right)$. Either $(u, v)$ or $(v, u)$ is not in $\alpha$. That edge must be in $\gamma_{1}$, as it is in $\beta=\alpha \vee \gamma_{1}$, and $\{u, v\}$ is a $\beta$-block. Similarly, it must be in $\gamma_{2}$, which contradicts $\gamma_{1} \wedge \gamma_{2} \leq \alpha$.

Now assume $\alpha^{*}=\beta^{*}$. In this case, by Proposition 5.8 and Theorem 5.17, $\beta \backslash \alpha$ must be a single edge. This is impossible: by $\alpha \vee \gamma_{1}=\alpha \vee \gamma_{2}=\beta$, both $\gamma_{1}$ and $\gamma_{2}$ must contain this edge, but then $\gamma_{1} \wedge \gamma_{2} \not \leq \alpha$.

Problem 6.37. Is it true that any algebra in a congruence join semidistributive variety is quasiorder join semidistributive? If not, is it true with the assumption that the variety is locally finite? Is it true that if the congruence lattices are all join semidistributive over modular, then so are the quasiorder lattices?

We refer to [19] and Chapter 9 of [21] for more on the join semidistributive over modular property for congruence lattices. As it relates to the solvability relation, the expected answer to the last question is "no". For other reasons (mainly to do with the proof of Theorem 6.32) we also expect "no" to be the answer to the other question.

Lemma 6.38. Suppose $\mathbf{L}$ is a lattice, and $\theta$ is a congruence on $\mathbf{L}$ such that all $\theta$-blocks have at most two elements. Then if $\mathbf{L}$ is join semidistributive, then so is $\mathbf{L} / \theta$.

Proof. Suppose there are elements $a, b, c \in L$ so that

$$
(a \vee b) / \theta=(a \vee c) / \theta>(a \vee(b \wedge c)) / \theta .
$$

By the join semidistributivity of $\mathbf{L}, a \vee b=a \vee c$ is impossible, but $a \vee b$ and $a \vee c$ are in the same (necessarily two-element) $\theta$-block. It may be assumed that $a \vee c \prec a \vee b$.

We claim that

$$
a \vee((a \vee c) \wedge b)=a \vee c
$$


It is obvious that $\leq$ is satisfied in the above equality, but also, the two sides are in the same $\theta$-block by

$$
a \vee((a \vee c) \wedge b) \stackrel{\theta}{\leftrightarrow} a \vee((a \vee b) \wedge b)=a \vee b \stackrel{\theta}{\leftrightarrow} a \vee c,
$$

and $a \vee c$ is the smallest element of that $\theta$-block.

Now, the join semidistributivity of $\mathbf{L}$ implies

$$
a \vee c=a \vee(c \wedge(a \vee c) \wedge b)=a \vee(b \wedge c),
$$

which contradicts $(a \vee c) / \theta>(a \vee(b \wedge c)) / \theta$.

Lemma 6.39. Suppose $\mathbf{L}$ is a finite lattice, and $\theta$ is a congruence on $\mathbf{L}$ so that all $\theta$-blocks have at most two elements. If $\mathbf{L}$ is join semidistributive and $\mathbf{L} / \theta$ is lower bounded, then $\mathbf{L}$ is also lower bounded.

Proof. Let $\mathbf{L}^{\prime}:=\mathbf{L} / \theta$, and denote with $K$ the set of elements of $L^{\prime}$ corresponding to two-element $\theta$-classes. For any $k \in K$, there are elements $k_{b} \prec k_{t}$ of $\mathbf{L}$ such that $k_{b} / \theta=k_{t} / \theta=k$. Introduce the binary relation $\rightsquigarrow$ on $K$ with

$$
k^{(1)} \rightsquigarrow k^{(2)} \Leftrightarrow\left(k_{t}^{(1)} \vee k_{b}^{(2)}=k_{t}^{(2)}\right) \wedge\left(k_{t}^{(1)} \wedge k_{b}^{(2)}=k_{b}^{(1)}\right),
$$

this is a partial order on $K$. We denote with $\sim$ the equivalence relation generated by it.

Claim 1. $\sim=^{-1} \circ \rightsquigarrow$.

As $\rightsquigarrow$ is a partial order, it is enough to show $\rightsquigarrow^{-1} \circ \rightsquigarrow \subseteq \rightsquigarrow 0 \rightsquigarrow^{-1}$. Suppose that $k^{(1)} \rightsquigarrow k^{(2)} \rightsquigarrow^{-1} k^{(3)}$. By the join semidistributivity of $\mathbf{L}$ and

$$
k_{b}^{(2)} \vee k_{t}^{(1)}=k_{b}^{(2)} \vee k_{t}^{(3)}=k_{t}^{(2)},
$$

it follows that $k_{t}^{(1)} \wedge k_{t}^{(3)} \not \leq k_{b}^{(2)}$, thus

$$
k_{t}^{(1)} \wedge k_{t}^{(3)} \neq k_{b}^{(1)} \wedge k_{b}^{(3)} .
$$

This means that $k^{(1)} \wedge k^{(3)} \in K$, and it is easy to check that $k^{(1)} \rightsquigarrow^{-1} k^{(1)} \wedge$ $k^{(3)} \rightsquigarrow k^{(3)}$. The claim is proved.

Claim 2. For $k^{(1)}, k^{(2)} \in K, k^{(1)} \sim k^{(2)}$ is equivalent to $k^{(1)} \rightsquigarrow^{-1} k^{(1)} \wedge k^{(2)} \rightsquigarrow$ $k^{(2)}$.

By the previous claim, we only have to prove that if $k^{(1)} \rightsquigarrow^{-1} k^{(3)} \rightsquigarrow k^{(2)}$, then one can exchange $k^{(3)}$ into $k^{(1)} \wedge k^{(2)}$. By $k_{b}^{(3)} \leq k_{b}^{(1)}, k_{b}^{(2)}$, it follows that $k^{(3)} \leq k^{(1)} \wedge k^{(2)}$. But $k_{t}^{(3)} \not k_{b}^{(1)}, k_{b}^{(2)}$, which is only possible if $k_{b}^{(1)} \wedge k_{b}^{(2)}$ is the bottom element of a two-element $\theta$-class, whose top element is not lower than either $k_{b}^{(1)}$ or $k_{b}^{(2)}$. This means precisely that $k^{(1)} \rightsquigarrow^{-1} k^{(1)} \wedge k^{(2)} \rightsquigarrow k^{(2)}$.

Claim 3. The $\sim$-classes are lower pseudointervals in $L^{\prime}$. 
The previous claim shows that they are closed to meets, all we need to prove is that they are convex. Suppose that $k^{(1)} \leq l^{\prime} \leq k^{(2)}$, where $l^{\prime} \in L^{\prime}$, and $k^{(1)} \sim k^{(2)}$. Again by the previous claim, we get that there is an $h \in K$ so that $h_{t} \leq k_{t}^{(1)}$ and $h_{t} \not \leq k_{b}^{(2)}$, therefore $k_{t}^{(1)} \not \leq k_{b}^{(2)}$, and $k^{(1)} \rightsquigarrow k^{(2)}$. Hence, $l^{\prime} \in K$, because otherwise $k_{t}^{(1)}$ would be smaller then the (unique) element of $L$ corresponding to $l^{\prime}$, which would be smaller then $k_{b}^{(2)}$. Now if $k^{(1)} \not \rightarrow l^{\prime}$, then $k_{t}^{(1)} \leq l_{b}^{\prime}$, but then also $k_{t}^{(1)} \leq k_{b}^{(2)}$, a contradiction. Therefore, $l^{\prime}$ is in the $\sim$-class of $k^{(1)}$, and the claim is proved.

As the $\sim$-classes are obviously disjoint, by Theorem 2.32 we only have to prove that when doubling the $\sim$-classes in $\mathbf{L}^{\prime}$, one gets a lattice isomorphic to L. To do that, check that for $k^{(1)} \sim k^{(2)}$,

$$
k_{b}^{(1)} \leq k_{b}^{(2)} \Leftrightarrow k^{(1)} \leq k^{(2)} \Leftrightarrow k_{t}^{(1)} \leq k_{t}^{(2)} \Leftrightarrow k_{b}^{(1)} \leq k_{t}^{(2)}
$$

and $k_{t}^{(1)} \leq k_{b}^{(2)}$ is impossible (by Claim 2), and if for $l_{1}, l_{2} \in L, l_{1} / \theta \nsim l_{2} / \theta$, then

$$
l_{1} \leq l_{2} \Leftrightarrow l_{1} / \theta \leq l_{2} / \theta
$$

Lemma 6.40. Suppose $\mathbf{L}$ is a finite join semidistributive lattice, and $\theta$ is a congruence on $\mathbf{L}$ so that all $\theta$-blocks having at least three elements are isomorphic to the direct square of the two-element lattice. If $\theta$ is an atom in $\mathbf{C o n} \mathbf{L}$, then no block of it contains more than two elements.

Proof.

Claim 1. Suppose that $a \prec b$ with $a$ and $b$ being in the same $\theta$-block. Then $a \vee c \preceq b \vee c$ and $a \wedge c \preceq b \wedge c$ for any $c \in L$.

Assume first that $a \vee c<x, y<b \vee c$ (the case when $a \vee c$ and $b \vee c$ are not in a one- or two-element $\theta$-block). As neither $x$ nor $y$ can be larger than (or equal to) $b$,

$$
x \vee y=x \vee b=b \vee c>x=x \vee a=x \vee(y \wedge b),
$$

contradicting join semidistributivity. Likewise, if $a \wedge c<x, y<b \wedge c$, then neither $x$ nor $y$ can be smaller than (or equal to) $a$, and

$$
a \vee x=a \vee y=b>a=a \vee(a \wedge c)=a \vee(x \wedge y)
$$

contradicts join semidistributivity. The claim is proved.

Introduce the relation $\rightsquigarrow$ on the set of those prime quotients of $\mathbf{L}$ whose two elements are in the same $\theta$-block with

$$
\left(x_{1}, x_{2}\right) \rightsquigarrow\left(y_{1}, y_{2}\right) \Leftrightarrow\left(x_{2} \vee y_{1}=y_{2}\right) \wedge\left(x_{2} \wedge y_{1}=x_{1}\right)
$$

This is a preorder.

Claim 2. The transitive closure of this relation is $\sim:=^{-1} \circ \rightsquigarrow$. 
Suppose that

$$
\left(x_{1}, x_{2}\right) \rightsquigarrow\left(y_{1}, y_{2}\right) \rightsquigarrow^{-1}\left(z_{1}, z_{2}\right),
$$

and let us show that

$$
\left(x_{1}, x_{2}\right) \rightsquigarrow^{-1}\left(x_{1} \wedge z_{1}, x_{2} \wedge z_{2}\right) \rightsquigarrow\left(z_{1}, z_{2}\right) .
$$

By the join semidistributivity of $\mathbf{L}$ it follows that $x_{2} \wedge z_{2} \not \leq y_{1}$, otherwise

$$
y_{1} \vee x_{2}=y_{1} \vee z_{2}=y_{2}>y_{1}=y_{1} \vee\left(x_{2} \wedge z_{2}\right),
$$

hence $x_{1} \wedge z_{1} \neq x_{2} \wedge z_{2}$. By the previous claim,

$$
x_{1} \wedge z_{1} \preceq x_{1} \wedge z_{2}, x_{2} \wedge z_{1} \preceq x_{2} \wedge z_{2},
$$

so if $x_{1} \wedge z_{1}$ and $x_{2} \wedge z_{2}$ were respectively the bottom and the top element of a $\theta$-class, then the other two elements would have to be $x_{1} \wedge z_{2}$ and $x_{2} \wedge z_{1}$ (because $x_{1} \wedge z_{2}=x_{2} \wedge z_{1}>x_{1} \wedge z_{1}$ is clearly not the case). But that would mean that

$$
\left(x_{1} \wedge z_{2}\right) \vee\left(x_{2} \wedge z_{1}\right)=x_{2} \wedge z_{2},
$$

which contradicts $x_{2} \wedge z_{2} \not \leq y_{1}$. Thus, $\left(x_{1} \wedge z_{1}, x_{2} \wedge z_{2}\right)$ is a prime quotient in a $\theta$-block, and as $x_{2} \wedge z_{2} \leq x_{1}$ or $x_{2} \wedge z_{2} \leq z_{1}$ would mean $x_{2} \wedge z_{2} \leq y_{1}$, the claim is proved.

Now assume that there is a $\theta$-block with elements $a<b, c<d$. As $\theta$ is an atom in Con $\mathbf{L}$, it is the congruence generated by the edge $(a, b)$. The set

$$
\rho:=\left\{(x, y) \in L^{2}: x=y \text { or }(x, y) \sim(a, b)\right\}
$$

is closed under the unary polynomials of $\mathbf{L}$ by Claim 1 : for any $(x, y) \in \rho$ any any $z, x \vee z \prec y \vee z$ means $(x, y) \rightsquigarrow(x \vee z, y \vee z)$ and $x \wedge z \prec y \wedge z$ means $(x, y) \rightsquigarrow^{-1}(x \wedge z, y \wedge z)$. Therefore, $\theta$ is the symmetric and transitive closure of $\rho$. This is only possible if $(b, d) \in \rho$ (because if $(a, c) \in \rho$, then by $(a, c) \rightsquigarrow(b, d)$ one sees that $(b, d)$ is also in $\rho)$.

By Claim 2, there is a prime quotient $(e, f)$ in $\mathbf{L}$ so that $(a, b) \rightsquigarrow^{-1}(e, f)$ and $(e, f) \rightsquigarrow(b, d)$. The first relation implies that $f \leq b$, the second that $f \vee b=d$, this contradiction finishes the proof.

Lemma 6.41. Suppose $\mathbf{L}$ is a finite lattice, and $\theta$ is a congruence on $\mathbf{L}$ such that all $\theta$-blocks having at least three elements are isomorphic to the direct square of the two-element lattice. If $\mathbf{L}$ is join semidistributive and $\mathbf{L} / \theta$ is lower bounded, then $\mathbf{L}$ is also lower bounded.

Proof. Suppose that in the congruence lattice of $\mathbf{L}$,

$$
0_{\mathbf{L}} \prec \theta_{1} \prec \cdots \prec \theta_{k}=\theta .
$$

We use induction on $k$. The case $k=1$ is an obvious consequence of Lemmas 6.39 and 6.40 . 
If $k>1$, then by Lemmas 6.38 and $6.40, \mathbf{L} / \theta_{1}$ is a join semidistributive lattice. Notice that $\theta / \theta_{1}$ is a congruence of $\mathbf{L} / \theta_{1}$ that does not have a block with more than two elements that is not isomorphic with the direct square of the two-element lattice. Hence by the inductive hypothesis $\mathbf{L} / \theta_{1}$ is a lower bounded lattice, and then so is $\mathbf{L}$, because the $k=1$ case of the lemma is already proved, and it can be applied for $\theta_{1}$ in the place of $\theta$.

Theorem 6.42. Suppose that $\mathbf{A}$ is a finite algebra in a variety omitting 1, 2, and 5. Then Quo $\mathbf{A}$ is a lower bounded lattice.

Proof. We use induction on $|A|$.

For any prime quotient $\alpha \prec \beta$ in Quo $\mathbf{A}$ such that $\mathbf{A}$ is not $(\alpha, \beta)$-minimal, take an $(\alpha, \beta)$-minimal set $M$. The algebra $\left.\mathbf{A}\right|_{M}$ is in a variety omitting 1,2 , and 5 by Proposition 4.26 and Theorem 4.29, thus by the inductive hypothesis Quo $\left.\mathbf{A}\right|_{M}$ is a lower bounded lattice. By Proposition 4.3 and Theorem 4.11, the restriction to $M$ is a lattice homomorphism from Quo $\mathbf{A}$ onto Quo $\left.\mathbf{A}\right|_{M}$. Denote the kernel of this homomorphism with $\theta_{\alpha, \beta}$. Note that $\alpha$ and $\beta$ are in different $\theta_{\alpha, \beta}$-blocks, and that Quo $\mathbf{A} / \theta_{\alpha, \beta} \cong$ Quo $\left.\mathbf{A}\right|_{M}$ is a lower bounded lattice.

Introduce

$$
\theta:=\bigwedge_{\text {Ais not }(\alpha, \beta) \text {-minimal }} \theta_{\alpha, \beta} .
$$

As

$$
\text { Quo } \mathbf{A} / \theta \leq{ }_{S} \quad \prod \quad \text { Quo } \mathbf{A} / \theta_{\alpha, \beta},
$$

Ais not $(\alpha, \beta)$-minimal

$\mathbf{A} / \theta$ is a lower bounded lattice by Theorem 2.33. As for each $\alpha \prec \beta$ in Quo $\mathbf{A}$ such that $\mathbf{A}$ is not $(\alpha, \beta)$-minimal, $(\alpha, \beta) \notin \theta_{\alpha, \beta}$, each $\theta$-block is such that $\mathbf{A}$ is minimal to each prime quotient in it.

Consequently, $\mathbf{A}$ is minimal for any quasiorders $\gamma<\delta$ satisfying $(\gamma, \delta) \in \theta$. By Theorem 5.22, all $\theta$-blocks have either one or two elements, or are isomorphic to the lattice $\mathbf{2}^{2}$ (as $\mathbf{A}$ is in a variety omitting 1, 2, and 5). By Theorem 6.36, Quo $\mathbf{A}$ is a join semidistributive lattice. Thus by Lemma 6.41, Quo $\mathbf{A}$ is a lower bounded lattice.

Corollary 6.43. Suppose that $\mathcal{V}$ is a variety omitting 1, 2, and 5, and the finite algebras in the variety all have meet semidistributive quasiorder lattices. Then the quasiorder lattices of the finite algebras of $\mathcal{V}$ are all bounded lattices.

Proof. This is an immediate consequence of Theorem 6.42 and Day's Theorem (Theorem 2.64 of [10]) stating that if a lattice is semidistributive, then it is either neither lower nor upper bounded, or bounded.

\subsection{Quasiorder meet semidistributivity}

According to Theorem 6.36 , if a finite algebra generates a congruence join semidistributive variety, it is quasiorder join semidistributive. According to Theorem 6.7, this is also true for distributivity and modularity, and indeed any 
quasi-idenity stronger than modularity. But Theorem 6.22 shows that meet semidistributivity is an other matter. This raises the following question.

Problem 6.44. Characterize those varieties whose every algebra is quasiorder meet semidistributive. Characterize those varieties whose every finite algebra is quasiorder meet semidistributive.

There is reason to believe that these two questions have different answers: in the variety of semilattices, the congruence lattice of no finite algebra contains a sublattice isomorphic to $\mathbf{M}_{3}$ (Theorem 6.20 ), but there is an infinite algebra whose congruence lattice does (Theorem 6.32). Note that for locally finite varieties, not having a congruence lattice with a sublattice isomorphic to $\mathbf{M}_{3}$ is equivalent to congruence meet semidistributivity (Theorem 4.28).

Conjecture 6.45. Suppose that $\mathbf{A}$ is a finite algebra such that Quo $\mathbf{A}$ does not omit 5 . Then there is a finite algebra in the variety generated by $\mathbf{A}$ whose quasiorder lattice is not meet semidistributive.

If this conjecture is true, that will mean that quasiorder meet semidistributivity is a consequence of join semidistributivity for finite algebras of a variety. For congruences, the implication goes the other way (see Theorems 4.28 and 4.29). It would, of course, still be possible that both implications hold, but while we believe that Conjecture 6.45 is true, we do not know whether finite algebras lying in a variety omitting 1,2 , and 5 need to have meet semidistributive quasiorder lattices. It may be that quasiorder meet semidistributivity is equivalent to quasiorder distributivity (for varieties). In any case, by Corollary 6.43 , it would follow from Conjecture 6.45 that quasiorder meet semidistributivity means quasiorder boundedness for finite algebras of a variety. 


\section{Chapter 7}

\section{Summary}

This thesis is about quasiorders of algebras. A quasiorder is a preorder (reflexive and transitive binary relation) on the underlying set of the algebra that is compatible with the algebra's operations. The quasiorders of an algebra $\mathbf{A}$ form a lattice with respect to inclusion, denoted by Quo $\mathbf{A}$.

For a trivial algebra, each preorder is a quasiorder. Lattices of preorders are quite complicated in the following sense: each lattice can be embedded into a preorder lattice (of a large enough underlying set), moreover, this can be done so the embedding is into a principal ideal of the preorder lattice which is generated by an antisymmetric preorder. In other words, each lattice can be represented as a lattice of posets. It is not true, however, that each finite lattice is representable as a lattice of posets on a finite underlying set: this can only be done if the lattice is (McKenzie-) lower bounded [30, 2].

Lower boundedness is a property that can be checked for final lattices in polynomial (cube) time. If $a$ and $b$ are distinct join irreducible elements of the lattice $\mathbf{L}$, then $a$ and $b$ are said to be $D$-related $(a D b)$ if there is a $c \in L$ such that $a \leq b \vee c$ but $a \not \leq d \vee c$ for any $d<b$. A lattice is lower bounded if it is finally generated and the $D$ relation does not contain an infinite path.

In the thesis we first inquire about the following question: which lattices can be represented as a lattice of posets satisfying DCC (that is, posets not containing infinite descending chains). We give a necessary condition for this.

Definition 7.1. An element $c \in L$ is completely join irreducible if either it is the smallest element of $\mathbf{L}$ or there is a $c^{*} \in L$ that is the greatest of all elements of $\mathbf{L}$ smaller than $c$.

Definition 7.2. Let $\underline{a}:=a_{1} D a_{2} D \ldots D a_{k} D a_{1}$ and $\underline{b}:=b_{1} D b_{2} D \ldots D b_{l} D b_{1}$ $D$-circles in $\mathbf{L}$ consisting only of completely join irreducible elements. The circle $\underline{a}$ is said to depend on the circle $\underline{b}$ if there are indices $i$ and $j$ such that

- $a_{j+1} \leq b_{i} \vee a_{j}$,

- $a_{j+1} \not \leq b_{i} \vee a_{j}^{*}$, 
- $a_{j+1} \not \leq b_{i}^{*} \vee a_{j}$.

Theorem 3.7. If dependency, as a binary relation on the D-circles of $\mathbf{L}$ itself contains a circle, then $\mathbf{L}$ is not representable with posets satisfying DCC.

For finite lattices, we prove a sufficient and necessary condition. It shows that deciding whether a finite lattice can be represented by posets satisfying DCC is in $\mathcal{E} \mathcal{X} \mathcal{P} \mathcal{T} \mathcal{M} \mathcal{E}$ (and probably in $\mathcal{N} \mathcal{P}$ ).

Definition 7.3. For a finite lattice $\mathbf{L}, \mathcal{C}_{\mathbf{L}}$ denotes the set of nontrivial join covers of join irreducibles, i.e. the set

$$
\begin{aligned}
& \left\{\left(l, l_{1}, \ldots, l_{k}\right) \in J(L)^{k+1}: l \leq l_{1} \vee l_{2} \vee \cdots \vee l_{k},\right. \\
& \left.l \not \leq\left(l_{1}\right)^{*} \vee l_{2} \vee \cdots \vee l_{k}, l \not \leq l_{1} \vee\left(l_{2}\right)^{*} \vee \cdots \vee l_{k}, \ldots, l \not \leq l_{1} \vee l_{2} \vee \cdots \vee\left(l_{k}\right)^{*}\right\}
\end{aligned}
$$

Theorem 3.4. A finite lattice $\mathbf{L}$ is representable with DCC posets if and only if there is a mapping $s: \mathcal{C}_{\mathbf{L}} \mapsto L$ satisfying the following:

- for any $\left(l, l_{1}, \ldots, l_{k}\right) \in \mathcal{C}_{\mathbf{L}}, s\left(l, l_{1}, \ldots, l_{k}\right) \in\left\{l_{1}, \ldots, l_{k}\right\}$,

- $s$ is symmetrical in all but the first variable, i.e. for any permutation $\pi \in S_{k}$,

$$
s\left(l, l_{1}, \ldots, l_{k}\right)=s\left(l, l_{\pi(1)}, \ldots, l_{\pi(k)}\right),
$$

- for the binary relations

$$
T_{\mathbf{L}}:=\left\{\left(l, l_{i}\right):\left(l, l_{1}, \ldots, l_{k}\right) \in \mathcal{C}_{\mathbf{L}}, s\left(l, l_{1}, \ldots, l_{k}\right) \neq l_{i}\right\}
$$

and

$U_{\mathbf{L}}:=\operatorname{Tr}\left(\{(l, l): l \in L\} \cup\left\{\left(l, l_{i}\right):\left(l, l_{1}, \ldots, l_{k}\right) \in \mathcal{C}_{\mathbf{L}}, s\left(l, l_{1}, \ldots, l_{k}\right)=l_{i}\right\}\right)$, the relation $U_{\mathbf{L}} \circ T_{\mathbf{L}}$ does not contain a circle.

There is a related question explored in the thesis: which lattices can be embedded into quasiorder lattices of semilattices? The main result: the lattice $\mathbf{M}_{3}$ is embeddable (while it is not embeddable into a congruence lattice of a semilattice). The construction can be extended to any (potentially infinite) lattice to give a homomorphism of the lattice to the quasiorder lattice of a semilattice. Our (unproven) conjecture is that this homomorphism is always an embedding.

The remaining parts of the thesis are universal algebra. The main question: in what way can the Hobby-McKenzie tame congruence theory [21] be extended to quasiorders, and how far that extension can be taken? Tame congruence theory gives a type from 1 to 5 to every covering pair of a congruence lattice (Con A) of a finite algebra A. This so-called "labeling" has the following properties:

- if $\theta \in \operatorname{Con} \mathbf{A}$, then the types of covering pairs in $\operatorname{Con}(\mathbf{A} / \theta)$ are the same as the types of the corresponding pairs in Con $\mathbf{A}$ (pairs over the congruence $\theta)$, 
- if the covering pairs $\alpha \prec \beta$ and $\gamma \prec \delta$ are prime perspective, that is, $\beta \wedge \gamma=\alpha$ and $\beta \vee \gamma=\delta$, then the types of the two pairs are the same,

- any type depends only on the polynomial clone of the algebra, and not on the principal operations or the terms,

- the solvability relation on Con $\mathbf{A}$ ( $\mu$ and $\nu$ are in the same class if the interval $[\mu \wedge \nu, \mu \vee \nu]$ does not contain types 3, 4, and 5) is a lattice congruence, and factoring out with it gives an upper bounded lattice.

A major part in the definition of types is a "descent" to a minimal algebra (with respect to the given congruence pair). We will not give details here, only mention that this can be done to quasiorder pairs without trouble, and the resulting minimal algebra will be essentially unique.

We give a definition of types for covering pairs of the quasiorder lattice. This still has all but the last of the preceding properties, and is really an extension: if a covering quasiorder pair consists of two congruences, then the pair has the same congruence and quasiorder types. The following method will give the type of a covering edge $(\alpha, \beta)$ of Quo A:

- "Descend" to an $(\alpha, \beta)$-minimal algebra. From now on, $\mathbf{A}$ is assumed to be $(\alpha, \beta)$-minimal, so any non-bijective polynomial maps $\beta$-edges into $\alpha$-edges.

- Take the congruences $\alpha^{*}:=\alpha \wedge \alpha^{-1}$ and $\beta^{*}:=\beta \wedge \beta^{-1}$. First assume that $\alpha^{*} \neq \beta^{*}$. If $\beta^{*}$ covers $\alpha^{*}$ in the congruence lattice, then $(\alpha, \beta)$ will inherit the congruence type of $\left(\alpha^{*}, \beta^{*}\right)$. Otherwise, the type of $(\alpha, \beta)$ will be 1.

- From now on, $\alpha^{*}=\beta^{*}$. We define $\mathbf{A}_{+}$, the enlargement of $\mathbf{A}$ by $\beta$. It consists of those elements $(a, b, c) \in A^{3}$ satisfying $(a, b),(b, c) \in \beta$. (So it is a subalgebra of $\mathbf{A}^{3}$ ).

- For every $\delta \in$ Quo $\mathbf{A}$, there is a congruence $\delta_{+} \in$ Con $\mathbf{A}_{+}$defined as the transitive closure of the following relation:

$$
\left\{\left((a, b, c),\left(a, b^{\prime}, c\right)\right) \in \mathbf{A}_{+}^{2}:\left(b, b^{\prime}\right) \in \delta \cup \delta^{-1}\right\} .
$$

- It can be proved that $\alpha_{+}$and $\beta_{+}$are different congruences of $\mathbf{A}_{+}$. Let us list which (congruence) types are there in the interval $\left[\alpha_{+}, \beta_{+}\right]$. If there is a type 4 , then the quasiorder pair $(\alpha, \beta)$ will have type 4 . If there is no type 4 in the interval, but there is type 5 , then $(\alpha, \beta)$ will have type 5 . In all other cases, the type of $(\alpha, \beta)$ will be 1 .

This definition has the following important properties:

Theorem 6.1. For any $i \in\{1,2,3,4,5\}$ and any variety $\mathcal{V}, \mathcal{V}$ omits $i$ for congruences iff it omits $i$ for quasiorders.

Theorem 5.18. Prime perspective quasiorder pairs have the same type. 
The important property not necessarily true for quasiorder lattices is that the solvability relation is a congruence. For this we give a four-element semigroup as an example. This example also shows that there is no way to define solvability in an other way so it is a congruence on the quasiorder lattice and an extension of the solvability relation of the congruence lattice. On the other hand, if the algebra is finite and generates a congruence modular variety, then the solvability relation is a congruence on the quasiorder lattice, and factoring out with it yields a distributive lattice.

We have studied quasiorder lattices in locally finite varieties omitting certain types. We want to highlight the following theorems:

Theorem 6.7. Suppose that $\mathbf{A}$ is a finite algebra in a congruence modular variety. Then $\mathbf{Q u o} \mathbf{A}$ and $\mathbf{C o n} \mathbf{A}$ satisfy the same lattice identities.

Theorem 6.20. If $\mathbf{A}$ is a finite algebra, and $\operatorname{HSP}(\mathbf{A})$ is a variety omitting 1, then Quo $\mathbf{A}$ does not contain a nonmodular simple sublattice. Furthermore, if the variety also omits 2, then Quo $\mathbf{A}$ does not contain any simple sublattice other than the trivial and the two-element lattice.

Theorem 6.42. If $\mathbf{A}$ is a finite algebra, and $\operatorname{HSP}(\mathbf{A})$ is a variety omitting 1,2, and 5, then Quo $\mathbf{A}$ is a lower bounded lattice. 


\section{Chapter 8}

\section{Összefoglaló}

A disszertáció algebrák kvázirendezéseiről szól. Kvázirendezésnek nevezzük egy algebra alaphalmazának olyan preorderjeit (reflexív és tranzitív binér reláci-óit), melyek kompatibilisek az algebra alapműveleteivel. Egy A algebra kvázirendezései a tartalmazásra nézve hálót alkotnak, ezt Quo A-val jelöljük.

Triviális algebra esetén minden preorder kvázirendezés. A preorderhálók elég bonyolultak a következő értelemben: minden háló beágyazható (elég nagy alaphalmazhoz tartozó) preorderhálóba, sőt, az is elérhető, hogy a preorderháló egy (lineáris) rendezés által generált főideáljába történjen a beágyazás [1]. Ezt úgy is mondhatjuk, hogy minden háló reprezentálható poset-ek hálójaként. Nem igaz azonban, hogy minden véges háló reprezentálható véges alaphalmazon megadott poset-ek hálójaként: egy véges háló csak akkor reprezentálható így, ha (McKenzie-) alulról korlátos [30, 2].

Az alulról korlátosság a (véges) hálók jól (köbös időben) ellenőrizhető tulajdonsága. Ha $a$ és $b$ az $\mathbf{L}$ háló két különböző egyesítés irreducibilis eleme, akkor azt mondjuk, hogy $a$ és $b D$-relációban vannak $(a D b)$, amennyiben van olyan $c \in L$, hogy $a \leq b \vee c$, de $a \not \leq d \vee c$ tetszőleges $d<b$ esetén. Egy háló alulról korlátos, ha végesen generált és a $D$-reláció nem tartalmaz végtelen hosszú utat.

A disszertációban először egy ehhez kapcsolódó kérdést vizsgálunk: milyen hálók reprezentálhatóak olyan poset-ekkel, amelyik teljesítik az ún. DCC tulajdonságot, vagyis nem tartalmaznak végtelen csökkenő láncot. Megadunk egy feltételt, melyet egy hálónak mindenképp teljesíteni kell, hogy reprezentálható lehessen így. Ez a feltétel a következő:

Definíció 8.1. Egy $c \in L$ teljesen egyesités irreducibilis, ha vagy az $\mathbf{L}$ legkisebb eleme, vagy létezik egy $c^{*} \in L$ elem, ami a legnagyobb a $c$-nél kisebb elemek között.

Definíció 8.2. Legyenek $\underline{a}:=a_{1} D \ldots D a_{k} D a_{1}$ és $\underline{b}:=b_{1} D \ldots D b_{l} D b_{1}$ csupa teljesen egyesítés irreducibilis elemekből álló D-körök L-ben. Azt mondjuk, hogy $\underline{a}$ függ $\underline{b}$-től, ha léteznek $i$ és $j$ indexek, melyekre:

- $a_{j+1} \leq b_{i} \vee a_{j}$, 
- $a_{j+1} \not \leq b_{i} \vee a_{j}^{*}$,

- $a_{j+1} \not \leq b_{i}^{*} \vee a_{j}$

Tétel 3.7. Ha a függőség, mint az $\mathbf{L}$ D-körein értelmezett binér reláció tartalmaz kört, akkor $\mathbf{L}$ nem reprezentálható a DCC-t teljesítö poset-ekkel.

Véges hálókra egy szükséges és elégséges feltételt bizonyítunk. Ez mutatja, hogy annak eldöntése, hogy egy háló reprezentálható-e DCC-t teljesítő posetekkel, $\mathcal{E} \mathcal{X} \mathcal{P} \mathcal{T} \mathcal{I} \mathcal{M E}$-ban (és valószínúleg $\mathcal{N} \mathcal{P}$-ben) van.

Definíció 8.3. Egy véges $\mathbf{L}$ háló esetén $\mathcal{C}_{\mathbf{L}}$ jelöli a minimális nemtriviális egyesítés irreducibilisek közötti fedéseket, vagyis a következö halmazt:

$$
\begin{aligned}
& \left\{\left(l, l_{1}, \ldots, l_{k}\right) \in J(L)^{k+1}: l \leq l_{1} \vee l_{2} \vee \cdots \vee l_{k},\right. \\
& \left.l \not \leq\left(l_{1}\right)^{*} \vee l_{2} \vee \cdots \vee l_{k}, l \not \leq l_{1} \vee\left(l_{2}\right)^{*} \vee \cdots \vee l_{k}, \ldots, l \not \leq l_{1} \vee l_{2} \vee \cdots \vee\left(l_{k}\right)^{*}\right\}
\end{aligned}
$$

Tétel 3.4. Egy véges $\mathbf{L}$ háló akkor és csak akkor reprezentálható DCC posetekkel, ha létezik egy $s: \mathcal{C}_{\mathbf{L}} \mapsto L$ leképezés, melyre:

- minden $\left(l, l_{1}, \ldots, l_{k}\right) \in \mathcal{C}_{\mathbf{L}}$ esetén $s\left(l, l_{1}, \ldots, l_{k}\right) \in\left\{l_{1}, \ldots, l_{k}\right\}$,

- s szimmetrikus az elsőt leszámítva az összes változójában, vagyis minden $\pi \in S_{k}$ permutációra

$$
s\left(l, l_{1}, \ldots, l_{k}\right)=s\left(l, l_{\pi(1)}, \ldots, l_{\pi(k)}\right),
$$

- $a$

$$
T_{\mathbf{L}}:=\left\{\left(l, l_{i}\right):\left(l, l_{1}, \ldots, l_{k}\right) \in \mathcal{C}_{\mathbf{L}}, s\left(l, l_{1}, \ldots, l_{k}\right) \neq l_{i}\right\}
$$

és

$U_{\mathbf{L}}:=\operatorname{Tr}\left(\{(l, l): l \in L\} \cup\left\{\left(l, l_{i}\right):\left(l, l_{1}, \ldots, l_{k}\right) \in \mathcal{C}_{\mathbf{L}}, s\left(l, l_{1}, \ldots, l_{k}\right)=l_{i}\right\}\right)$

binér relációkra az $U_{\mathbf{L}} \circ T_{\mathbf{L}}$ reláció nem tartalmaz kört.

A disszertáció egy másik, a fentiekhez közeli témával is foglalkozik: mely hálók ágyazhatók be félháló kvázirendezés-hálójába. A fó eredmény az, hogy az $\mathbf{M}_{3}$ háló beágyazható (félháló kongruenciahálójába ez a háló nem ágyazható be). A konstrukció kiterjeszthető tetszőleges (akár végtelen) hálóra, eredményül egy, a hálóból egy félháló kvázirendezés-hálójába menő homomorfizmust adva. (Bizonyítatlan) sejtésünk az, hogy ez a homomorfizmus mindig beágyazás.

A disszertáció további része univerzális algebra. A fó kérdés: miként és meddig lehet a Hobby-McKenzie féle TCT-t [21] kiterjeszteni kvázirendezésekre? A TCT egy véges $\mathbf{A}$ algebra kongruenciahálójának (Con $\mathbf{A}$ ) fedő párjaihoz rendel egy 1 és 5 közötti típust. Ez a hozzárendelés többek között a következő tulajdonságokkal rendelkezik:

- ha $\theta \in \operatorname{Con} \mathbf{A}$, akkor $\operatorname{Con}(\mathbf{A} / \theta)$ fedő párjainak típusa megegyezik az adott $\mathbf{A} / \theta$-beli kongruenciáknak A-ban megfelelő ( $\theta$ fölötti) kongruenciák által alkotott pár típusával, 
- ha az $\alpha \prec \beta$ és $\gamma \prec \delta$ fedő párok prím perspektívek, vagyis $\beta \wedge \gamma=\alpha$ és $\beta \vee \gamma=\delta$, akkor a két pár típusa megegyezik,

- a párok típusa csak az algebra polinomjainak klónjától függ, nem az algebra alapmüveleteitől vagy kifejezéseitől,

- a Con A-n értelmezett feloldhatósági (solvability) reláció ( $\mu$ és $\nu$ akkor van relációban, ha a $[\mu \wedge \nu, \mu \vee \nu]$ intervallum nem tartalmaz 3-as, 4-es, illetve 5-ös típust) egy kongruencia a kongruenciahálón, és a vele vett faktor egy felülról korlátos háló.

A típusok definíciójának lényeges eleme a "lemenetel" (egy adott párra nézve) minimális algebrára. Ezt nem részletezzük, de kvázirendezés párokra gond nélkül átvihető a fogalom, és a minimális algebra lényegében egyértelmü lesz.

Megadunk egy olyan definíciót kvázirendezés-hálók fedő párjainak típusára, amely a fentiek közül az összes tulajdonságot teljesíti az utolsó kivételével, és kiterjesztés: ha két kongruencia fedi egymást a kvázirendezés-hálóban, akkor a pár típusa ugyanaz marad. A következő eljárás dönti el egy Quo A-ban fedő $(\alpha, \beta)$ pár típusát:

- "Menjünk le" minimális algebrára. A továbbiakban A $(\alpha, \beta)$-minimális, vagyis minden nem bijektív egyváltozós polinom a $\beta$-éleket $\alpha$-élekbe viszi.

- Tekintsük az $\alpha^{*}:=\alpha \wedge \alpha^{-1}$ és a $\beta^{*}:=\beta \wedge \beta^{-1}$ kongruenciákat. Tegyük fel, hogy $\alpha^{*} \neq \beta^{*}$. Ha $\beta^{*}$ fedi $\alpha^{*}$-ot a kongruenciahálóban, akkor $(\alpha, \beta)$ örökölje $\left(\alpha^{*}, \beta^{*}\right)$ típusát. Ha nincs fedés, legyen $(\alpha, \beta)$ típusa 1.

- A továbbiakban $\alpha^{*}=\beta^{*}$. Definiáljuk $\mathbf{A}_{+}$-ot, $\mathbf{A} \beta$-val vett felfúltját, ami $A^{3}$ azon $(a, b, c)$ elemeiból áll, melyekre $(a, b)$ és $(b, c) \beta$-beli (ez egy részalgebrája $\mathbf{A}^{3}$-nek).

- Minden $\delta \in$ Quo A-nak megfeleltetünk egy $\delta_{+} \in$ Con $\mathbf{A}_{+}$-ot, amit úgy definiálunk, mint a következő reláció tranzitív lezártját:

$$
\left\{\left((a, b, c),\left(a, b^{\prime}, c\right)\right) \in \mathbf{A}_{+}^{2}:\left(b, b^{\prime}\right) \in \delta \cup \delta^{-1}\right\} .
$$

- Bizonyítható, hogy $\alpha_{+}$és $\beta_{+}$különböző kongruenciák $\mathbf{A}_{+}$-ban. Nézzük meg, milyen (kongruencia) típusok vannak $\left[\alpha_{+}, \beta_{+}\right]$-ban. Ha van 4 -es típus, akkor az $(\alpha, \beta)$ kvázirendezés párnak a típusa is 4 lesz. Ha nincs 4 -es, de van 5 -ös, akkor $(\alpha, \beta)$ típusa is 5 . Minden más esetben $(\alpha, \beta)$ típusa 1.

A definíciónak fontos tulajdonságai az alábbiak:

Tétel 6.1. Legyen $\mathcal{V}$ egy varietás, $i \in\{1,2,3,4,5\}$. $\mathcal{V}$ véges algebráinak kongruencia típusai között akkor és csak akkor jelenik meg az $i$ típus, ha a kvázirendezés tipusok között is megjelenik.

Tétel 5.18. Prím pepspektív kvázirendezés párok típusa megegyezik. 
A lényeges tulajdonság, ami nem feltétlen teljesül kvázirendezés-hálokra, az a feloldhatósagi reláció kongruencia volta. Erre egy négyelemü félcsoportot hozunk példának. Ez a példa azt is mutatja, hogy nem lehet a feloldhatóságot máshogy sem definiálni úgy, hogy az kongruencia legyen a kvázirendezés-hálón, és kiterjesztése legyen a kongruencia feloldhatósági relációnak. Ellenben ha egy algebra véges és kongruencia moduláris varietást generál, akkor a feloldhatósági reláció kongruencia a kvázirendezés-hálón, és a vele vett faktor egy disztributív háló.

Vizsgáltuk azt, hogy mit lehet mondani egy adott típust nem tartalmazó, lokálisan véges varietás kvázirendezés-hálóiról. A következő tételeket kívánjuk kiemelni:

Tétel 6.7. Tegyük fel, hogy $\mathbf{A}$ egy véges algebra, amely kongruencia moduláris varietást generál. Ekkor Quo $\mathbf{A}$ és Con $\mathbf{A}$ ugyanazokat a hálóazonosságokat elégítik $k i$.

Tétel 6.20. Ha $\mathbf{A}$ egy véges algebra, és $\operatorname{HSP}(\mathbf{A})$ egy 1-es típust nem tartalmazó varietás, akkor Quo $\mathbf{A}$ nem tartalmaz nemmoduláris egyszerü részhálót. Amennyiben a varietás nem tartalmaz 2-es típust sem, akkor Quo A semmilyen egyszerü részhálót sem tartalmaz, leszámítva a triviális és a kételemü hálót.

Tétel 6.42. Ha $\mathbf{A}$ egy véges algebra, és $\operatorname{HSP}(\mathbf{A})$ egy 1-es, 2-es, és 5-ös típust nem tartalmazó varietás, akkor Quo A alulról korlátos háló. 


\section{Acknowledgements}

The topic of this thesis was suggested by my advisor, Miklós Maróti. I am very grateful for this suggestion, as it was very rewarding to work in this area, and I sincerely believe that there are serious depths to be explored yet. I also recieved a crucial help from him for dealing with +-quotients, without which this thesis would be either shallower, uglier, or nonexisting.

I would also thank my high school mathematics teacher, Emese Osváth, who made me love maths more than I could reasonably love anything to do with "science", and the Bolyai Institute, where I recieved my professional education. In particular, the algebra department provided me a quality postgradual education-all but one of my doctoral courses provided me with knowledge that is used in this thesis, and even the one exception has some relevance. 


\section{Bibliography}

[1] B.M. Achein: A representation theorem for lattices, Algebra universalis 2 (1972), pages $177-178$.

[2] K.V. Adaricheva, V.A. Gurbunov and V.I. Tumanov: Join-semidistributive lattices and convex geometries, Advances in Mathematics, 173 (1) (2003), pages $1-49$.

[3] S. Burris and H.P. Sankappanavar: A course in universal algebra, Graduate Texts in Mathematics, 78, Springer-Verlag, New York-Berlin (1981).

[4] N. Caspard: The Lattice of Permutations Is Bounded, International Journal of Algebra and Computation, 08/2000; 10 (4) (2000), pages 481-490.

[5] G. Czédli, E. K. Horváth and P. Lipparini: Optimal Mal'tsev conditions for congruence modular varieties, Algebra Universalis 53 (2-3) (2005), pages 267-279.

[6] G. Czédli: A Horn sentence for involution lattices of quasiorders, Order, 11 (1994) pages 391-395.

[7] G. Czédli and L. Szabó: Quasiorders of lattices versus pairs of congruences, Acta Sci. Math. (Szeged), 60 (1995), pages 207-211.

[8] A. Day: A characterizations of modularity for congruence lattices of algebras, Canad. Math. Bull. 12 (1969), pages 167-173.

[9] A. Day: Characterizations of finite lattices that are bounded-homomorphic images of sublattices of free lattices, Canad. J. Math. 31 (1979), pages 6978.

[10] R. Freese, J. Ježek and J. B. Nation: Free Latices, Mathematical Surveys and Monographs, vol. 42, Amer. Math. Soc., Providence, RI, 1995.

[11] R. Freese and J.B. Nation: Congruence lattices of semilattices, Pacific Journal of Mathematics 49 (1) (1973), pages 51-58.

[12] R. Freese, K. Kearnes and J. B. Nation: Congruence lattices of congruence semidistributive algebras, Lattice Theory and Applications, Research and Exposition in Mathematics, vol. 23, Heldermann, Lemgo, 1995, pages 6378. 
[13] P.A. Grillet: Semigroups: An Introduction to the Structure Theory, Dekker, New York, 1995.

[14] H.-P. Gumm: Congruence modularity is permutability composed with distributivity, Archiv der Math. (Basel) 36 (1981), 569-576.

[15] G. Gyenizse: On lattice representations with posets, submitted to Order.

[16] G. Gyenizse: Quasiorder lattices in congruence modular varieties, submitted to Acta Sci. Math. (Szeged).

[17] G. Gyenizse: Types of quasiorders, draft.

[18] G. Gyenizse and M. Maróti: Quasiorder lattices of varieties, to appear in Algebra Universalis.

[19] G. Gyenizse, M. Maróti and L. Zádori: The structure of polynomial operations associated with smooth digraphs, Algebra Universalis 72 (4) (2014), pages 381-391.

[20] J. Hagemann and A. Mitschke: On n-permutable congruences, Algebra Universalis 3 (1973), pages 8-12.

[21] D. Hobby and R. McKenzie: The Structure of Finite Algebras, Memoirs of the American Mathematical Society, No. 76, American Mathematical Society, Providence, RI, 1988.

[22] B. Jónsson: Congruence varieties, Algebra Universalis 10 (1980), pages 355-394.

[23] A. Kazda, M. Kozik, R. McKenzie and M. Moore: Absorption and directed Jónsson terms, arXiv:1502.01072 [math.RA], 4 Feb 2015, 17 pages.

[24] M. Kozik, A. Krokhin, M. Valeriote and R. Willard: Characterizations of several Maltsev conditions, Algebra universalis 73 (3-4) (2015), pages 205-224.

[25] R. McKenzie: Equational bases and non-modular lattice varieties, Trans. Amer. Math. Soc., 174 (1972), pages 1-43.

[26] R. McKenzie and J. Snow: Congruence modular varieties: commutator theory and its uses, Structural Theory of Automata, Semigroups, and Universal Algebra, NATO Science Series, pages 273-329 (2003).

[27] P.P. Pálfy: Unary polynomials in algebras I, Algebra universalis 18 (1984), pages $262-273$.

[28] P. Pudlák and J. Tůma: Every finite lattice can be embedded in the lattice of all equivalences over a finite set, Algebra Universalis 10 (1980), pages 74-95. 
[29] M. V. Semenova: Lattices that Are Embeddable in Suborder Lattices, Algebra and Logic, 44 (4) (2005), pages 270-285.

[30] B. Sivak: Representation of finite lattices by orders on finite sets, Mathematica Slovaca, 28 (2) (1978), pages 203-215.

[31] P. M. Whitman: Lattices, equivalence relations, and subgroups, Bull. Amer. Math. Soc. 52 (1946), pages 507-522. 